\title{
TOTAL SYNTHESIS OF THE POLYENE-POLYOL MACROLIDE RK-397, FEATURING CROSS-COUPLINGS OF ALKYNYLEPOXIDE MODULES
}

\author{
Svetlana A. Burova and Frank E. McDonald* \\ Department of Chemistry, Emory University, 1515 Pierce Drive, Atlanta, GA 30322
}

\section{SUPPORTING INFORMATION}

Complete experimental details and compound characterizations, crystallographic data for a derivative of compound 23, discussion of attempted hydration from iodocyclization of the tert-butylcarbonate derivative of $\mathbf{3 8}$, and comparison of spectral data of synthetic and natural RK-397 (4) and tetraacetonide derivative 52.

General: ${ }^{1} \mathrm{H}$ NMR and ${ }^{13} \mathrm{C}$ NMR spectra were measured in $\mathrm{CDCl}_{3}$ on INOVA 400 or INOVA 600 NMR spectrometers. All proton NMR spectra were recorded at 400 or 600 $\mathrm{MHz}$ and were referenced with residual chloroform $(7.27 \mathrm{ppm})$ or residual methanol (4.87 ppm). All carbon NMR spectra were measured at 100 or $150 \mathrm{MHz}$ and were referenced with $77.2 \mathrm{ppm}$ resonance of residual chloroform. Infrared spectra were recorded on sodium chloride discs on Mattson Genesis II FT-IR. High - resolution FAB mass spectra were collected on JEOL SX102 at 6kV-Xe using 3-nitrobenzyl alcohol, in some cases with addition of Lil as a matrix. MALDI mass spectra were collected on Voyager-STR MALDI time-of flight mass spectrometer using 2, 5-dihydroxybenzoic acid. Elemental analyses were performed by Atlantic Microlab, Inc in Norcross, GA. Optical rotations were measured using a $10 \mathrm{~cm}$ cell with Perkin-Elmer Polarimeter $241 \mathrm{MC}$. All reactions were conducted in oven-dried $\left(125^{\circ} \mathrm{C}\right)$ glassware under an atmosphere of dry argon. All common reagents and solvents were obtained from commercial suppliers and used without further purification unless otherwise indicated. Solvents were dried by standard methods. Flash chromatography was carried out on standard grade silica gel $60 \AA, 32-63 \mu$. 
(3S,4S)-2,4-Dimethyl-3-(p-methoxybenzyloxy)-5-hexene (16): (3S,4S)-2,4Dimethylhex-5-en-3-ol ${ }^{1}(15,5.0 \mathrm{~g}, 75 \%$ pure, $29.3 \mathrm{mmol})$ and freshly prepared 4methoxybenzyltrichloroacetimidate ${ }^{2}$ were dissolved in $\mathrm{CH}_{2} \mathrm{Cl}_{2}(40 \mathrm{~mL})$, and 10camphorsulfonic acid $(930 \mathrm{mg}, 3.98 \mathrm{mmol})$ was added. The mixture was stirred for 16 $\mathrm{h}$, diluted with water and extracted with ether. The organic fractions were dried with $\mathrm{MgSO}_{4}$, filtered, concentrated and chromatographed with pentane - ether (98:2). PMBn ether 16 was isolated in $67 \%$ yield $(4.9 \mathrm{~g})$.

${ }^{1} \mathrm{H} \mathrm{NMR}\left(\mathrm{CDCl}_{3}, 600 \mathrm{MHz}\right): \delta 7.29(\mathrm{~d}, 2 \mathrm{H}, J=8.4 \mathrm{~Hz}), 6.88(\mathrm{~d}, 2 \mathrm{H}, J=8.4 \mathrm{~Hz}), 5.85(\mathrm{~m}$, $1 \mathrm{H}), 5.06(\mathrm{~d}, 1 \mathrm{H}, J=17.4 \mathrm{~Hz}), 4.98(\mathrm{~d}, 1 \mathrm{H}, J=9.0 \mathrm{~Hz}), 4.54(\mathrm{~d}, 1 \mathrm{H}, J=10.2 \mathrm{~Hz}), 4.49(\mathrm{~d}$, $1 \mathrm{H}, J=10.2 \mathrm{~Hz}), 3.81(\mathrm{~s}, 3 \mathrm{H}), 3.01(\mathrm{t}, 1 \mathrm{H}, J=5.4 \mathrm{~Hz}), 2.45(\mathrm{~m}, 1 \mathrm{H}), 1.87(\mathrm{~m}, 1 \mathrm{H}), 1.08$ $(\mathrm{d}, 3 \mathrm{H}, J=6.6 \mathrm{~Hz}), 0.96(\mathrm{~m}, 6 \mathrm{H}) .{ }^{13} \mathrm{C} \mathrm{NMR}\left(\mathrm{CDCl}_{3}, 150 \mathrm{MHz}\right): \delta$ 159.2, 142.9, 131.5, 129.4, 113.9, 113.8, 88.6, 75.0, 55.5, 41.3, 31.2, 20.6, 17.7, 15.6. HRMS (FAB+): Calcd. for $\mathrm{C}_{16} \mathrm{H}_{24} \mathrm{O}_{2} \mathrm{Li}\left([\mathrm{M}+\mathrm{Li}]^{+}\right)$, 255.1936. Found, 255.1937.

(4S,5S)-4,6-Dimethyl-5-(p-methoxybenzyloxy)-trans-hept-2-enoate, methyl ester (18). p-Methoxybenzyl ether $16(4.7 \mathrm{~g}, 19 \mathrm{mmol})$ and methyl acrylate $(5.1 \mathrm{~mL}, 57$ mmol) were dissolved in $\mathrm{CH}_{2} \mathrm{Cl}_{2}(15 \mathrm{~mL})$, and (4,5-dihydrolMes) $\mathrm{Cl}_{2} \mathrm{Ru}=\mathrm{CH}-(0-\mathrm{O} P r) \mathrm{C}_{6} \mathrm{H}_{4}$ $(25,250 \mathrm{mg}, 42 \mu \mathrm{mol})$ was added as a solid in one portion. The reaction was refluxed for $5 \mathrm{~h}$ then stirred for 2 days at ambient temperature and concentrated. The residue was chromatographed with pentane - ether $(92.5: 7.5)$ producing $58 \%$ yield $(3.4 \mathrm{~g})$ of 18.

${ }^{1} \mathrm{H}$ NMR $\left(\mathrm{CDCl}_{3}, 600 \mathrm{MHz}\right): \delta 7.27(\mathrm{~d}, 2 \mathrm{H}, J=7.8 \mathrm{~Hz}), 7.01(\mathrm{dd}, 1 \mathrm{H}, J=15.6 ; 5.4 \mathrm{~Hz})$, $6.78(\mathrm{~d}, 2 \mathrm{H}, J=7.8 \mathrm{~Hz}), 5.86(\mathrm{~d}, 1 \mathrm{H}, J=15.6 \mathrm{~Hz}), 4.48(\mathrm{~s}, 2 \mathrm{H}), 3.81(\mathrm{~s}, 3 \mathrm{H}), 3.75(\mathrm{~s}, 3 \mathrm{H})$, $3.08(\mathrm{t}, 1 \mathrm{H}, J=5.4 \mathrm{~Hz}), 2.62(\mathrm{~m}, 1 \mathrm{H}), 1.82(\mathrm{~m}, 1 \mathrm{H}), 1.12(\mathrm{~d}, 3 \mathrm{H}, J=6.6 \mathrm{~Hz}), 0.96(\mathrm{~m}, 6 \mathrm{H})$.

1. (a) Dreher, S. D.; Leighton, J. L J. Am. Chem. Soc. 2001, 123, 341. (b) Brown, H. C.; Bhat, K. S. J. Am. Chem. Soc. 1986, 108, 5919. (c) For ent-15, see: Ghosh, A. K.; Liu, W. J. Org. Chem. 1997, 62, 7908.

2. Preparation of 4-methoxybenzyltrichloroacetimidate: 4-methoxybenzyl alcohol $(5.0 \mathrm{~mL}, 40$ $\mathrm{mmol})$ was dissolved in ether $(15 \mathrm{~mL})$, and $\mathrm{NaH}(60 \%$ in mineral oil, $400 \mathrm{mg}$, ca. $10.0 \mathrm{mmol})$ was added. The reaction was stirred for 30 min then cooled to $0^{\circ} \mathrm{C}$, and $\mathrm{Cl}_{3} \mathrm{CCN}(4.0 \mathrm{~mL}, 40$ $\mathrm{mmol})$ was slowly added. The mixture was slowly warmed to room temperature and stirred for $1.5 \mathrm{~h}$, then diluted with saturated $\mathrm{NaHCO}_{3}$ and extracted with ether. The organic fractions were dried with $\mathrm{MgSO}_{4}$, filtered and concentrated producing crude 4methoxybenzyltrichloroacetimidate (ca. $39 \mathrm{mmol}$ ). 
${ }^{13} \mathrm{C} \mathrm{NMR}\left(\mathrm{CDCl}_{3}, 150 \mathrm{MHz}\right): \delta 167.4,159.3,152.9,131.0,129.5,120.2,113.9,87.6$, 75.0, 55.5, 51.7, 40.0, 31.5, 20.5, 17.7, 14.9. IR (neat, $\mathrm{cm}^{-1}$ ): 2960 (s, br), 2870 (s, br), 1725 (s), 1655 (s), 1630 (s), 1515 (s), 1465 (s), 1435 (s), 1300 (s), 1250 (s, br), 1175 (s, br), 1060 (s), 990 (s). HRMS (FAB+): Calcd. for $\mathrm{C}_{18} \mathrm{H}_{26} \mathrm{O}_{4}\left([\mathrm{M}]^{+}\right), 306.1831$. Found, 306.1856. Anal. Calcd. for $\mathrm{C}_{18} \mathrm{H}_{26} \mathrm{O}_{4}$ : $\mathrm{C}, 70.56$; $\mathrm{H}, 8.55$. Found: $\mathrm{C}, 70.26, \mathrm{H}, 8.66$. $[\alpha]_{D}$ +15 (c $\left.0.990, \mathrm{CHCl}_{3}\right)$.

(4S,5S)-4,6-Dimethyl-5-(p-methoxybenzyloxy)-trans-hept-2-enal (20): $1 \mathrm{M}$ solution of DIBAL-H in $\mathrm{CH}_{2} \mathrm{Cl}_{2}(32.4 \mathrm{~mL}, 32.4 \mathrm{mmol})$ was added dropwise to a solution of methyl ester $18(3.3 \mathrm{~g}, 10.8 \mathrm{mmol})$ in $\mathrm{CH}_{2} \mathrm{Cl}_{2}(50 \mathrm{~mL})$ at $-78^{\circ} \mathrm{C}$. The mixture was stirred at $-78^{\circ} \mathrm{C}$ for $30 \mathrm{~min}$, then quenched with anhydrous EtOAc $(35 \mathrm{~mL})$. After additional stirring at $-78^{\circ} \mathrm{C}$ for $30 \mathrm{~min}$, the reaction mixture was poured into saturated aqueous solution of sodium- potassium tartrate and stirred $16 \mathrm{~h}$ at room temperature. The layers were separated aqueous fractions were extracted with ether and ethyl acetate. Combined organic fractions were dried with $\mathrm{MgSO}_{4}$, filtered, concentrated and chromatographed with pentane - ether $(8: 2)$ to yield $(4 S, 5 S)-4,6$-dimethyl-5-( $p$ methoxybenzyloxy)-trans-hept-2-en-1-ol (2.73 g, 91\% yield).

${ }^{1} \mathrm{H}$ NMR $\left(\mathrm{CDCl}_{3}, 600 \mathrm{MHz}\right): \delta 7.28(\mathrm{~d}, 2 \mathrm{H}, J=8.4 \mathrm{~Hz}), 6.88(\mathrm{~d}, 2 \mathrm{H}, J=8.4 \mathrm{~Hz}), 5.78(\mathrm{~m}$, 2H), $4.51(\mathrm{~s}, 2 \mathrm{H}), 4.10(\mathrm{~d}, 2 \mathrm{H}, J=4.8 \mathrm{~Hz}), 3.81(\mathrm{~s}, 3 \mathrm{H}), 2.99(\mathrm{t}, 1 \mathrm{H}, J=6.0 \mathrm{~Hz}), 2.47(\mathrm{~m}$, $1 \mathrm{H}), 1.85(\mathrm{~m}, 1 \mathrm{H}), 1.07(\mathrm{~d}, 3 \mathrm{H}, J=6.6 \mathrm{~Hz}), 0.96(\mathrm{~m}, 6 \mathrm{H}) .{ }^{13} \mathrm{C} \mathrm{NMR}\left(\mathrm{CDCl}_{3}, 150 \mathrm{MHz}\right): \delta$ 159.2, 136.8, 131.4, 129.4, 128.3, 113.8, 88.5, 75.0, 64.0, 55.5, 39.8, 31.2, 20.6, 17.7 , 15.9. IR (neat, $\mathrm{cm}^{-1}$ ): 3390 (m, br), 2960 (s, br), 2870 (s, br), 1610 (s), 1510 (s), 1465 (m), $1380(\mathrm{~m}), 1300(\mathrm{~m}), 1250$ (s), $1170(\mathrm{~m}), 1080$ (s, br), 1040 (s, br), $955(\mathrm{~m}), 820$ (m). HRMS (FAB+): Calcd. for $\mathrm{C}_{17} \mathrm{H}_{26} \mathrm{O}_{3} \mathrm{Li}\left([\mathrm{M}+\mathrm{Li}]^{+}\right)$, 285.2042. Found, 285.2029. Anal. Calcd. for $\mathrm{C}_{17} \mathrm{H}_{26} \mathrm{O}_{3}$ : C, 73.34; $\mathrm{H}, 9.41$. Found: $\mathrm{C}, 73.24, \mathrm{H}, 9.53$. $[\alpha]_{D}+8$ (c 1.000, $\left.\mathrm{CHCl}_{3}\right)$.

(4S,5S)-4,6-Dimethyl-5-(p-methoxybenzyloxy)-trans-hept-2-en-1-ol (2.67 g, $9.63 \mathrm{mmol})$ and $\mathrm{Et}_{3} \mathrm{~N}(8.75 \mathrm{~mL})$ were dissolved in $\mathrm{CH}_{2} \mathrm{Cl}_{2}(40 \mathrm{~mL})$ and cooled to $0^{\circ} \mathrm{C}$. A solution of $\mathrm{SO}_{3}$-pyridine complex $(4.6 \mathrm{~g}, 28.9 \mathrm{mmol})$ in DMSO $(20 \mathrm{~mL})$ was added slowly at $0^{\circ} \mathrm{C}$. After addition, the reaction was stirred at ambient temperature for $4 \mathrm{~h}$, then diluted with 
saturated aqueous $\mathrm{NaHCO}_{3}$ and extracted with $\mathrm{CH}_{2} \mathrm{Cl}_{2}$ and $\mathrm{Et}_{2} \mathrm{O}$. The organic fractions were dried with $\mathrm{MgSO}_{4}$, filtered and concentrated. Aldehyde 20 (2.37 g, 89\% yield) was purified by flash chromatography with a mixture of pentane - ether $(8: 2)$.

${ }^{1} \mathrm{H}$ NMR $\left(\mathrm{CDCl}_{3}, 600 \mathrm{MHz}\right): \delta 9.47(\mathrm{~d}, 1 \mathrm{H}, J=7.8 \mathrm{~Hz}), 7.26(\mathrm{~d}, 2 \mathrm{H}, J=8.4 \mathrm{~Hz}), 6.88(\mathrm{~d}$, $2 \mathrm{H}, J=8.4 \mathrm{~Hz}$ ), $6.84(\mathrm{dd}, 1 \mathrm{H}, J=16.2 ; 7.8 \mathrm{~Hz}), 6.12(\mathrm{dd}, 1 \mathrm{H}, J=16.2 ; 8.4 \mathrm{~Hz}), 4.54(\mathrm{~d}$, $1 \mathrm{H}, J=10.8 \mathrm{~Hz}), 4.46(\mathrm{~d}, 1 \mathrm{H}, J=10.8 \mathrm{~Hz}), 3.81(\mathrm{~s}, 3 \mathrm{H}), 3.14(\mathrm{t}, 1 \mathrm{H}, J=5.4 \mathrm{~Hz}), 2.75(\mathrm{~m}$, $1 \mathrm{H}), 1.85(\mathrm{~m}, 1 \mathrm{H}), 1.16(\mathrm{~d}, 3 \mathrm{H}, J=6.6 \mathrm{~Hz}), 0.98(\mathrm{~m}, 6 \mathrm{H}) \cdot{ }^{13} \mathrm{C} \mathrm{NMR}\left(\mathrm{CDCl}_{3}, 150 \mathrm{MHz}\right): \delta$ 194.4, 162.2, 159.4, 132.0, 130.8, 129.5, 113.9, 87.3, 74.9, 55.4, 40.2, 31.5, 20.4, 18.1, 14.4. IR (neat, $\mathrm{cm}^{-1}$ ): 2960 (s, br), 2875 (s, br), 2730 (w), 1690 (s), 1630 (m), 1610 (s), 1515 (s), 1465 (s), 1380 (w), 1300 (m), 1250 (s), 1175 (s), 1110-1035 (s, br), 980 (m), 825 (s). HRMS (FAB+): Calcd. for $\mathrm{C}_{17} \mathrm{H}_{24} \mathrm{O}_{3} \mathrm{Li}\left([\mathrm{M}+\mathrm{Li}]^{+}\right)$, 283.1885. Found, 283.1894. Anal. Calcd. for $\mathrm{C}_{17} \mathrm{H}_{24} \mathrm{O}_{3}$ : C, 73.88; $\mathrm{H}, 8.75$. Found: $\mathrm{C}, 73.97, \mathrm{H}, 8.87$. $[\alpha]_{D}+14$ (c $\left.1.040, \mathrm{CHCl}_{3}\right)$.

Diol (22). $\mathrm{Et}_{3} \mathrm{~N}(3.4 \mathrm{~mL}, 24.7 \mathrm{mmol})$ and $\mathrm{Et}_{2} \mathrm{O}(30 \mathrm{~mL})$ were added to a $1 \mathrm{M}$ hexane solution of freshly prepared (-)- $-\mathrm{lpc}_{2} \mathrm{BOTf}^{3}(25 \mathrm{~mL}, 25 \mathrm{mmol})$. The mixture was cooled to $-78^{\circ} \mathrm{C}$ and a solution of 4 -(trimethylsilyl)-3-butyn-2-one $(3.37 \mathrm{ml}, 20.5 \mathrm{mmol})$ in $\mathrm{Et}_{2} \mathrm{O}(6$ $\mathrm{mL}$ ) was slowly injected over $5 \mathrm{~min}$. After additional stirring for $3 \mathrm{~h}$ at $-78^{\circ} \mathrm{C}$, a solution of aldehyde $20(2.27 \mathrm{~g}, 8.22 \mathrm{mmol})$ in $\mathrm{Et}_{2} \mathrm{O}(6 \mathrm{~mL})$ was added via cannula to the freshly formed solution of enol borinate 21 . The reaction was slowly warmed to $-30^{\circ} \mathrm{C}$ and stirred for $17 \mathrm{~h}$, then it was cooled to $-70^{\circ} \mathrm{C}$ and solid $\mathrm{NaBH}_{4}(3.1 \mathrm{~g}, 82.2 \mathrm{mmol})$ was added in one portion followed by dropwise addition of methanol $(15 \mathrm{~mL})$. After $10 \mathrm{~h}$ at $-70^{\circ} \mathrm{C}$, the reaction was diluted with $\mathrm{AcOH}(9 \mathrm{~mL})$ and left stirring overnight at room temperature. $\mathrm{H}_{2} \mathrm{O}_{2}(15 \mathrm{~mL})$ and $3 \mathrm{~N} \mathrm{NaOH}$ were slowly (Caution! Exothermic reaction!) and simultaneously added to the reaction from two different syringes at $0^{\circ} \mathrm{C}$. After addition, the mixture was left for $10-20$ min at $0^{\circ} \mathrm{C}$, then for $5 \mathrm{~h}$ at room temperature. The reaction was quenched with a saturated aqueous solution of $\mathrm{Na}_{2} \mathrm{SO}_{3}$ at $0^{\circ} \mathrm{C}$ (Caution! Exothermic reaction!), then was extracted with ether, dried with $\mathrm{Na}_{2} \mathrm{SO}_{4}$. The

3. (a) Paterson, I.; Goodman, J. M. Tetrahedron Lett. 1989, 30, 997. (b) Paterson, I.; Goodman, J. M.; Lister, M. A.; Schumann, R. C.; McClure, C. K.; Norcross, R. D. Tetrahedron 1990, 46, 4663. 
reaction was filtered, concentrated and chromatographed with mixture of pentane ether $(75: 25 \rightarrow 70: 30)$ to yield diol 22 (3.3 g, 97\% yield).

${ }^{1} \mathrm{H}$ NMR $\left(\mathrm{CDCl}_{3}, 600 \mathrm{MHz}\right): \delta 7.28(\mathrm{~d}, 2 \mathrm{H}, J=8.4 \mathrm{~Hz}), 6.88(\mathrm{~d}, 2 \mathrm{H}, J=8.4 \mathrm{~Hz}), 5.69(\mathrm{dd}$, $1 \mathrm{H}, J=7.8 \mathrm{~Hz}$ ), $5.51(\mathrm{dd}, 1 \mathrm{H}, J=15.0,6.6 \mathrm{~Hz}), 4.60(\mathrm{dd}, 1 \mathrm{H}, J=8.4,4.2 \mathrm{~Hz}), 4.50(\mathrm{~s}$, 2H), $4.35(\mathrm{~m}, 1 \mathrm{H}), 3.81(\mathrm{~s}, 3 \mathrm{H}), 2.98(\mathrm{t}, 1 \mathrm{H}, J=5.4 \mathrm{~Hz}), 2.44(\mathrm{~m}, 1 \mathrm{H}), 1.97(\mathrm{~m}, 1 \mathrm{H}), 1.88$ $(\mathrm{m}, 1 \mathrm{H}), 1.84(\mathrm{~m}, 1 \mathrm{H}), 1.07(\mathrm{~d}, 3 \mathrm{H}, J=7.2 \mathrm{~Hz}), 0.95(\mathrm{t}, 6 \mathrm{H}, J=7.2 \mathrm{~Hz}) .{ }^{13} \mathrm{C} \mathrm{NMR}\left(\mathrm{CDCl}_{3}\right.$, $150 \mathrm{MHz}): \delta 159.2,136.0,131.4,131.2,129.4,113.9,106.1,89.9,88.5,75.0,72.3$, 62.3, 55.5, 44.4, 39.8, 31.2, 20.6, 17.8, 15.9, 0.0. IR (neat, $\mathrm{cm}^{-1}$ ): 3390 (s, br), 2960 (s, br), 2900 (s), 2870 (s), $2170(\mathrm{~m}), 1615(\mathrm{~s}), 1515(\mathrm{~s}), 1465$ (s), $1380(\mathrm{~m}), 1300(\mathrm{~m}), 1250$ (s), $1170(\mathrm{~m}), 1065$ (s, br), $980(\mathrm{~m}), 845$ (s), $760(\mathrm{~m})$. HRMS (FAB+): Calcd. for $\mathrm{C}_{17} \mathrm{H}_{24} \mathrm{O}_{4} \mathrm{SiLi}\left([\mathrm{M}+\mathrm{Li}]^{+}\right), 425.2699$. Found, 425.2701. Anal. Calcd. for $\mathrm{C}_{24} \mathrm{H}_{38} \mathrm{O}_{4} \mathrm{Si}: \mathrm{C}$,

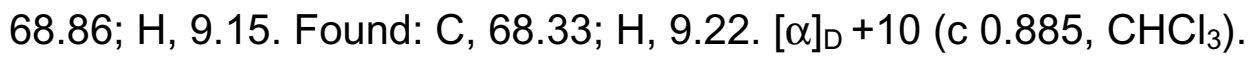

Monoacetonide 23. Diol $22(3.2 \mathrm{~g}, 7.66 \mathrm{mmol})$ was dissolved in methanol (15 mL), and $\mathrm{K}_{2} \mathrm{CO}_{3}(560 \mathrm{mg}, 4.0 \mathrm{mmol}$ ) was added, in order to remove the alkynyl trimethylsilyl group. The reaction was stirred at ambient temperature for $3 \mathrm{~h}$, then diluted with water and extracted with ether. The organic fractions were dried with $\mathrm{Na}_{2} \mathrm{SO}_{4}$, filtered and concentrated to give a crude sample of the terminal alkyne. This material was dissolved in 2,2-dimethoxypropane $(15 \mathrm{~mL}), \mathrm{TsOH}-\mathrm{H}_{2} \mathrm{O}(250 \mathrm{mg})$ and $3 \AA$ molecular sieves (300 $\mathrm{mg}$ ) were added. The mixture was stirred at room temperature for $17 \mathrm{~h}$, then diluted with $\mathrm{Et}_{3} \mathrm{~N}(1 \mathrm{~mL})$, filtered and concentrated. The residue was chromatographed with pentane-ether (85:15 $\rightarrow$ 8:2) to produce two fractions of diol 23: first fraction: dr, $1: 1.4$ (desired diastereomer as a minor compound) (360 mg, 12\%) and a more polar second fraction: dr, $6.25: 1$ (desired diastereomer as a major compound) $(2.22 \mathrm{~g}, 75 \%)$.

${ }^{1} \mathrm{H} \mathrm{NMR}\left(\mathrm{CDCl}_{3}, 600 \mathrm{MHz}\right): \delta 7.29(\mathrm{~d}, 2 \mathrm{H}, J=8.4 \mathrm{~Hz}), 6.88(\mathrm{~d}, 2 \mathrm{H}, J=8.4 \mathrm{~Hz}), 5.69$ (dd, $1 \mathrm{H}, J=15.6,7.8 \mathrm{~Hz}), 5.46(\mathrm{dd}, 1 \mathrm{H}, J=15.6,6.6 \mathrm{~Hz}), 4.69(\mathrm{~m}, 1 \mathrm{H}), 4.49(\mathrm{~s}, 2 \mathrm{H}), 4.31(\mathrm{~m}$, 1H), $3.81(\mathrm{~s}, 3 \mathrm{H}), 2.97(\mathrm{t}, 1 \mathrm{H}, J=6.0 \mathrm{~Hz}), 2.48(\mathrm{~s}, 1 \mathrm{H}), 2.45(\mathrm{~m}, 1 \mathrm{H}), 1.83(\mathrm{~m}, 1 \mathrm{H}), 1.73$ $(\mathrm{m}, 2 \mathrm{H}), 1.49(\mathrm{~s}, 3 \mathrm{H}), 1.48(\mathrm{~s}, 3 \mathrm{H}), 1.07(\mathrm{~d}, 3 \mathrm{H}, \mathrm{J}=6.6 \mathrm{~Hz}), 0.95(\mathrm{~d}, 6 \mathrm{H}, J=6.6 \mathrm{~Hz}) .{ }^{13} \mathrm{C}$ NMR $\left(\mathrm{CDCl}_{3}, 150 \mathrm{MHz}\right): \delta 159.2,136.8,131.3,129.5,129.0,113.8,99.6,88.5,82.6$, 75.0, 73.0, 69.8, 60.2, 55.4, 39.7, 37.5, 31.2, 30.3, 20.5, 19.6, 17.9, 15.4. IR (neat, $\mathrm{cm}^{-1}$ ): 3285 (m), 2960 (s), 2935 (s), 2870 (s), 1615 (s), 1515 (s), 1465 (s), 1380 (s), 
1300 (s), 1250 (s), 1200 (s), 1700 (s), 1085 (s), 970 (s), 920 (m), 875 (m), 825 (s). HRMS (FAB+): Calcd. for $\mathrm{C}_{24} \mathrm{H}_{34} \mathrm{O}_{4} \mathrm{Li}\left([\mathrm{M}+\mathrm{Li}]^{+}\right)$, 393.2617. Found, 393.2625. Anal. Calcd. for $\mathrm{C}_{24} \mathrm{H}_{34} \mathrm{O}_{4}$ : C, 74.58; $\mathrm{H}, 8.87$. Found: C, 74.46; $\mathrm{H}, 8.97$. $[\alpha]_{D}+36$ (c 0.740 , $\left.\mathrm{CHCl}_{3}\right)$.

Structure determination by crystallography: Compound 23 was subjected to standard oxidative deprotection of the $\mathrm{PMBn}$ ether with $\mathrm{DDQ}$ in wet $\mathrm{CH}_{2} \mathrm{Cl}_{2}$. A suitable crystal of the C31-alcohol corresponding to deprotection of 23 was coated with Paratone $\mathrm{N}$ oil, suspended in small fiber loop and placed in a coated nitrogen stream at $173 \mathrm{~K}$ on a Bruker D8 SMART 1000 CCD sealed tube diffractometer with graphite monochromated $\mathrm{CuK}_{\alpha}(1.54178 \AA)$ radiation. Data were measured using series of combination of phi and omega scans with 10 seconds frame exposures. There were two unique molecules in the asymmetric unit of the cell that were the same in chemical composition, but different in their orientation of the terminal chain group, the $\mathrm{CH}(\mathrm{OH})$ $\mathrm{CH}\left(\mathrm{CH}_{3}\right)_{2}$ end groups were rotated by $120^{\circ} \mathrm{C}$ about $\mathrm{C}(7)-\mathrm{C}(8)$ bond in the two different conformers.

Thermal ellipsoid diagram:

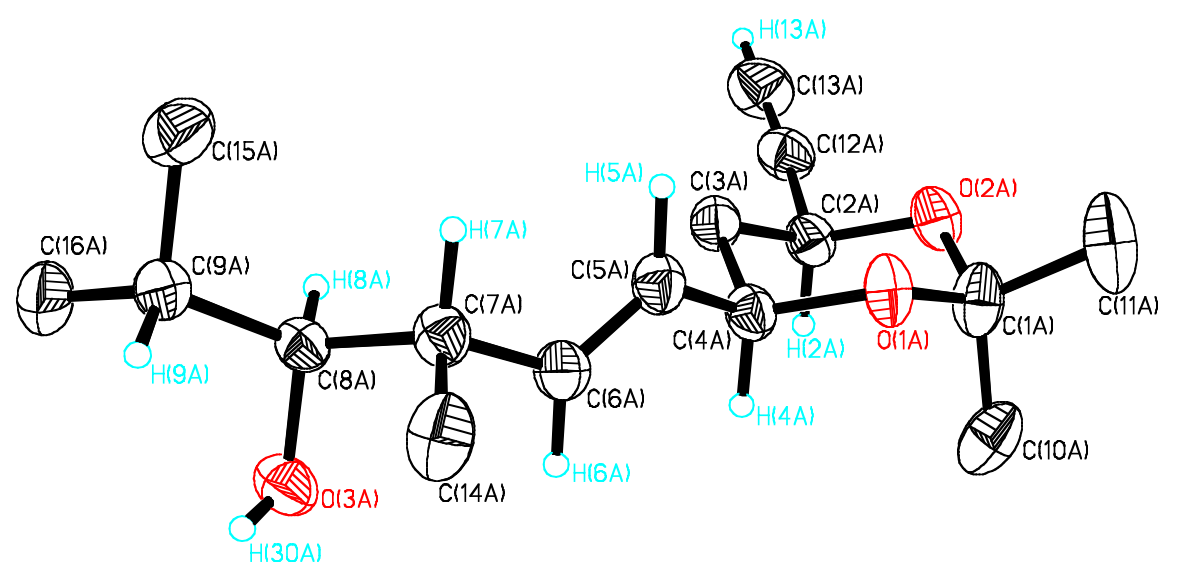


Table 1. Crystal data and structure refinement.

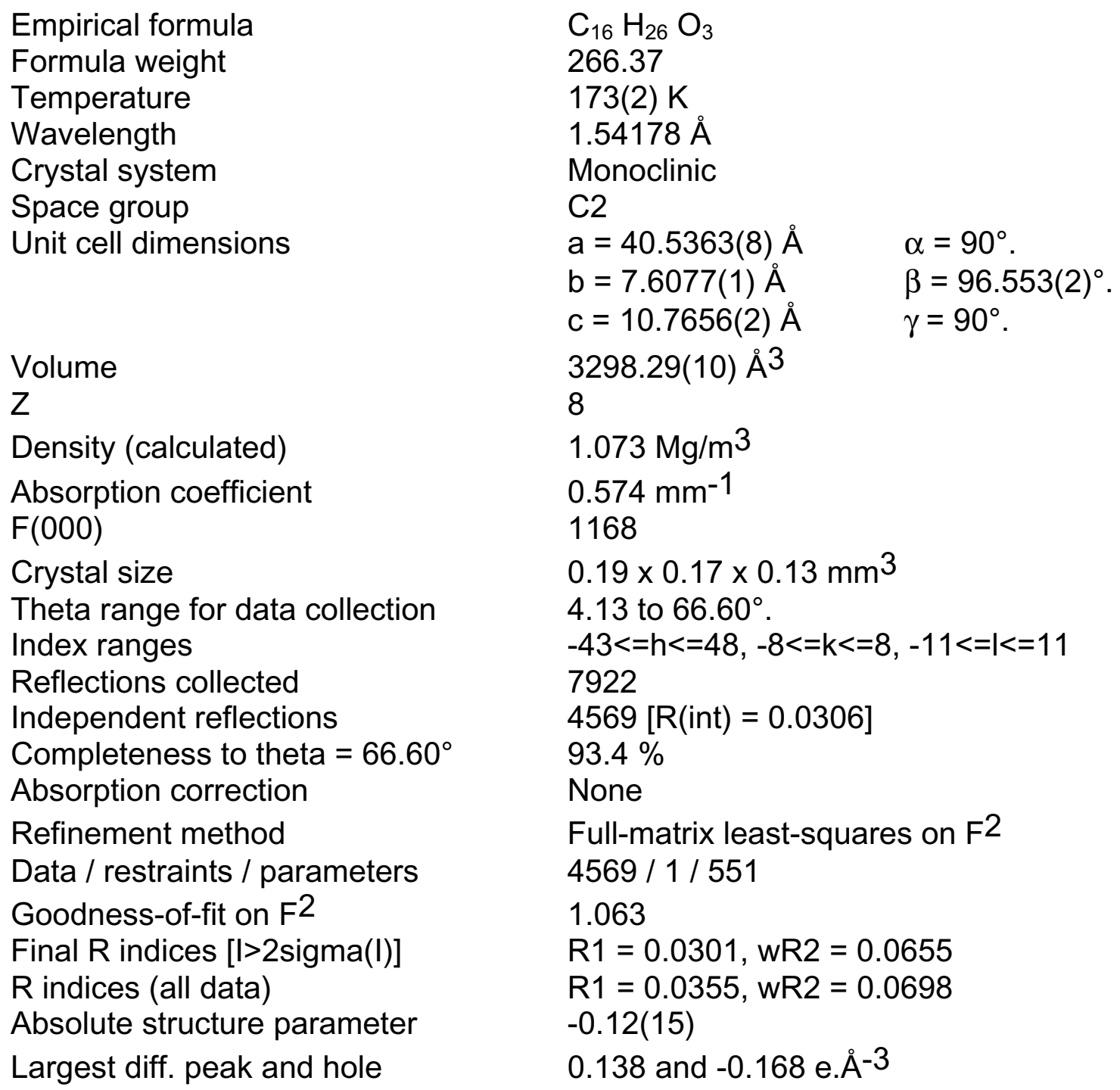

Table 2. Atomic coordinates ( $\times 10^{4}$ ) and equivalent isotropic displacement parameters $\left(\AA^{2} \times 10^{3}\right) . U(e q)$ is defined as one third of the trace of the orthogonalized Uij tensor.

\begin{tabular}{|c|c|c|c|c|}
\hline & $x$ & $y$ & $z$ & $\mathrm{U}(\mathrm{eq})$ \\
\hline $\mathrm{C}(1 \mathrm{~A})$ & $1867(1)$ & $8635(3)$ & $5067(2)$ & $39(1)$ \\
\hline$C(2 A)$ & $1903(1)$ & $6379(3)$ & $6650(2)$ & $32(1)$ \\
\hline$C(3 A)$ & $1696(1)$ & $5169(3)$ & $5740(2)$ & $32(1)$ \\
\hline$C(4 A)$ & $1469(1)$ & $6257(3)$ & $4808(2)$ & $31(1)$ \\
\hline$C(5 A)$ & $1300(1)$ & $5072(3)$ & $3821(2)$ & $33(1)$ \\
\hline$C(6 A)$ & 979(1) & $4742(3)$ & $3671(2)$ & $32(1)$ \\
\hline$C(7 A)$ & 812(1) & $3351(3)$ & 2831(2) & $32(1)$ \\
\hline$C(8 A)$ & 664(1) & 1958(3) & $3651(2)$ & 29(1) \\
\hline
\end{tabular}




\begin{tabular}{|c|c|c|c|c|}
\hline$C(9 A)$ & $531(1)$ & 295(3) & $2979(2)$ & $34(1)$ \\
\hline$C(10 A)$ & $1659(1)$ & 9914(3) & $5725(2)$ & $50(1)$ \\
\hline$C(11 A)$ & 2099(1) & $9540(4)$ & 4279(3) & $60(1)$ \\
\hline$C(12 A)$ & 2147(1) & $5375(3)$ & $7472(2)$ & $39(1)$ \\
\hline$C(13 A)$ & 2331(1) & 4485(3) & $8112(2)$ & $52(1)$ \\
\hline$C(14 A)$ & $554(1)$ & 4126(3) & $1843(2)$ & $44(1)$ \\
\hline$C(15 A)$ & 791(1) & $-563(3)$ & $2265(2)$ & $46(1)$ \\
\hline$C(16 A)$ & $415(1)$ & $-988(3)$ & 3913(3) & $51(1)$ \\
\hline$O(1 A)$ & 1661(1) & $7523(2)$ & 4228(1) & $36(1)$ \\
\hline$O(2 A)$ & 2077(1) & $7601(2)$ & $5947(1)$ & $37(1)$ \\
\hline$O(3 A)$ & $417(1)$ & $2772(2)$ & $4310(1)$ & $39(1)$ \\
\hline$C(1 B)$ & $8237(1)$ & $9060(3)$ & $9(2)$ & $33(1)$ \\
\hline$C(2 B)$ & $8189(1)$ & $6484(3)$ & $-1290(2)$ & $31(1)$ \\
\hline$C(3 B)$ & $8426(1)$ & $5500(3)$ & $-335(2)$ & $32(1)$ \\
\hline$C(4 B)$ & 8661(1) & $6812(3)$ & $387(2)$ & $29(1)$ \\
\hline$C(5 B)$ & $8865(1)$ & $5969(3)$ & 1478(2) & $33(1)$ \\
\hline$C(6 B)$ & 9187(1) & $5722(2)$ & 1584(2) & $30(1)$ \\
\hline$C(7 B)$ & 9391(1) & $4879(3)$ & 2678(2) & $31(1)$ \\
\hline $\mathrm{C}(8 \mathrm{~B})$ & $9567(1)$ & $3220(3)$ & 2288(2) & $29(1)$ \\
\hline $\mathrm{C}(9 \mathrm{~B})$ & 9338(1) & $1824(3)$ & 1632(2) & $36(1)$ \\
\hline$C(10 B)$ & $8413(1)$ & $10210(3)$ & $-869(2)$ & $42(1)$ \\
\hline$C(11 B)$ & $8022(1)$ & $10132(4)$ & $770(2)$ & $48(1)$ \\
\hline$C(12 B)$ & $7937(1)$ & $5309(3)$ & $-1934(2)$ & $39(1)$ \\
\hline$C(13 B)$ & $7747(1)$ & $4342(3)$ & $-2467(2)$ & $55(1)$ \\
\hline$C(14 B)$ & $9645(1)$ & $6202(3)$ & $3280(3)$ & $49(1)$ \\
\hline$C(15 B)$ & 9094(1) & 1092(4) & 2473(3) & $53(1)$ \\
\hline$C(16 B)$ & $9542(1)$ & 358(3) & 1138(2) & $53(1)$ \\
\hline $\mathrm{O}(1 \mathrm{~B})$ & $8470(1)$ & $8170(2)$ & 891(1) & $33(1)$ \\
\hline $\mathrm{O}(2 \mathrm{~B})$ & $8021(1)$ & $7827(2)$ & $-671(1)$ & $33(1)$ \\
\hline$O(3 B)$ & 9768(1) & $2462(2)$ & $3334(1)$ & $39(1$ \\
\hline
\end{tabular}

Table 3. Bond lengths $[\AA \AA]$ and angles $\left[{ }^{\circ}\right]$

\begin{tabular}{llll}
\hline $\mathrm{C}(1 \mathrm{~A})-\mathrm{O}(1 \mathrm{~A})$ & $1.433(2)$ & $\mathrm{C}(4 \mathrm{~A})-\mathrm{O}(1 \mathrm{~A})$ & $1.428(2)$ \\
$\mathrm{C}(1 \mathrm{~A})-\mathrm{O}(2 \mathrm{~A})$ & $1.433(2)$ & $\mathrm{C}(4 \mathrm{~A})-\mathrm{C}(5 \mathrm{~A})$ & $1.498(2)$ \\
$\mathrm{C}(1 \mathrm{~A})-\mathrm{C}(11 \mathrm{~A})$ & $1.503(3)$ & $\mathrm{C}(5 \mathrm{~A})-\mathrm{C}(6 \mathrm{~A})$ & $1.316(2)$ \\
$\mathrm{C}(1 \mathrm{~A})-\mathrm{C}(10 \mathrm{~A})$ & $1.517(3)$ & $\mathrm{C}(6 \mathrm{~A})-\mathrm{C}(7 \mathrm{~A})$ & $1.502(2)$ \\
$\mathrm{C}(2 \mathrm{~A})-\mathrm{O}(2 \mathrm{~A})$ & $1.433(2)$ & $\mathrm{C}(7 \mathrm{~A})-\mathrm{C}(14 \mathrm{~A})$ & $1.524(3)$ \\
$\mathrm{C}(2 \mathrm{~A})-\mathrm{C}(12 \mathrm{~A})$ & $1.465(3)$ & $\mathrm{C}(7 \mathrm{~A})-\mathrm{C}(8 \mathrm{~A})$ & $1.543(3)$ \\
$\mathrm{C}(2 \mathrm{~A})-\mathrm{C}(3 \mathrm{~A})$ & $1.525(3)$ & $\mathrm{C}(8 \mathrm{~A})-\mathrm{O}(3 \mathrm{~A})$ & $1.435(2)$ \\
$\mathrm{C}(3 \mathrm{~A})-\mathrm{C}(4 \mathrm{~A})$ & $1.525(3)$ & $\mathrm{C}(8 \mathrm{~A})-\mathrm{C}(9 \mathrm{~A})$ & $1.525(3)$
\end{tabular}




\begin{tabular}{|c|c|c|c|}
\hline$C(9 A)-C(16 A)$ & $1.515(3)$ & $C(6 A)-C(7 A)-C(8 A)$ & $108.48(15)$ \\
\hline$C(9 A)-C(15 A)$ & $1.518(3)$ & $C(14 A)-C(7 A)-C(8 A)$ & $112.61(16)$ \\
\hline$C(12 A)-C(13 A)$ & 1.171(3) & $O(3 A)-C(8 A)-C(9 A)$ & $111.28(15)$ \\
\hline $\mathrm{C}(1 \mathrm{~B})-\mathrm{O}(2 \mathrm{~B})$ & $1.428(2)$ & $O(3 A)-C(8 A)-C(7 A)$ & 108.96(15) \\
\hline$C(1 \mathrm{~B})-O(1 \mathrm{~B})$ & $1.429(2)$ & $C(9 A)-C(8 A)-C(7 A)$ & $115.97(16)$ \\
\hline$C(1 \mathrm{~B})-\mathrm{C}(11 \mathrm{~B})$ & $1.505(3)$ & $C(16 A)-C(9 A)-C(15 A)$ & $110.2(2)$ \\
\hline$C\left(1 B^{\prime}\right)-C(10 B)$ & $1.523(3)$ & $C(16 A)-C(9 A)-C(8 A)$ & $109.82(17)$ \\
\hline $\mathrm{C}(2 \mathrm{~B})-\mathrm{O}(2 \mathrm{~B})$ & $1.433(2)$ & $C(15 A)-C(9 A)-C(8 A)$ & 111.61(17) \\
\hline$C(2 B)-C(12 B)$ & $1.470(3)$ & $C(13 A)-C(12 A)-C(2 A)$ & $176.0(2)$ \\
\hline$C(2 B)-C(3 B)$ & $1.522(3)$ & $C(4 A)-O(1 A)-C(1 A)$ & $115.48(13)$ \\
\hline$C(3 B)-C(4 B)$ & $1.529(3)$ & $C(2 A)-O(2 A)-C(1 A)$ & $114.55(13)$ \\
\hline$C(4 B)-O(1 B)$ & $1.435(2)$ & $O(2 B)-C(1 B)-O(1 B)$ & $110.38(15)$ \\
\hline$C(4 B)-C(5 B)$ & $1.501(2)$ & $O(2 \mathrm{~B})-\mathrm{C}(1 \mathrm{~B})-\mathrm{C}(11 \mathrm{~B})$ & $105.90(17)$ \\
\hline$C(5 B)-C(6 B)$ & $1.311(2)$ & $\mathrm{O}(1 \mathrm{~B})-\mathrm{C}(1 \mathrm{~B})-\mathrm{C}(11 \mathrm{~B})$ & $105.93(15)$ \\
\hline$C(6 B)-C(7 B)$ & $1.503(2)$ & $O(2 B)-C(1 B)-C(10 B)$ & $111.28(15)$ \\
\hline$C(7 B)-C(14 B)$ & $1.530(3)$ & $O(1 \mathrm{~B})-\mathrm{C}(1 \mathrm{~B})-\mathrm{C}(10 \mathrm{~B})$ & $111.44(16)$ \\
\hline$C(7 B)-C(8 B)$ & $1.531(3)$ & $C(11 B)-C(1 B)-C(10 B)$ & 111.64(19) \\
\hline $\mathrm{C}(8 \mathrm{~B})-\mathrm{O}(3 \mathrm{~B})$ & $1.434(2)$ & $\mathrm{O}(2 \mathrm{~B})-\mathrm{C}(2 \mathrm{~B})-\mathrm{C}(12 \mathrm{~B})$ & 108.21(15) \\
\hline$C(8 B)-C(9 B)$ & $1.530(3)$ & $O(2 B)-C(2 B)-C(3 B)$ & 109.46(15) \\
\hline$C(9 B)-C(16 B)$ & $1.520(3)$ & $C(12 B)-C(2 B)-C(3 B)$ & $111.75(17)$ \\
\hline$C(9 B)-C(15 B)$ & $1.520(3)$ & $C(2 \mathrm{~B})-\mathrm{C}(3 \mathrm{~B})-\mathrm{C}(4 \mathrm{~B})$ & 109.33(16) \\
\hline$C(12 B)-C(13 B)$ & $1.169(3)$ & $O(1 \mathrm{~B})-\mathrm{C}(4 \mathrm{~B})-\mathrm{C}(5 \mathrm{~B})$ & $106.59(14)$ \\
\hline$O(1 A)-C(1 A)-O(2 A)$ & $110.57(16)$ & $O(1 B)-C(4 B)-C(3 B)$ & $109.25(14)$ \\
\hline$O(1 A)-C(1 A)-C(11 A)$ & $105.71(16)$ & $C(5 B)-C(4 B)-C(3 B)$ & 111.97(16) \\
\hline$O(2 A)-C(1 A)-C(11 A)$ & $105.25(18)$ & $C(6 B)-C(5 B)-C(4 B)$ & $125.69(18)$ \\
\hline$O(1 A)-C(1 A)-C(10 A)$ & 110.97(17) & $C(5 B)-C(6 B)-C(7 B)$ & $125.58(18)$ \\
\hline$O(2 A)-C(1 A)-C(10 A)$ & $111.28(17)$ & $C(6 B)-C(7 B)-C(14 B)$ & 109.55(17) \\
\hline$C(11 A)-C(1 A)-C(10 A)$ & $112.8(2)$ & $C(6 B)-C(7 B)-C(8 B)$ & $111.61(15)$ \\
\hline$O(2 A)-C(2 A)-C(12 A)$ & $108.58(15)$ & $C(14 B)-C(7 B)-C(8 B)$ & $110.30(16)$ \\
\hline$O(2 A)-C(2 A)-C(3 A)$ & $108.71(15)$ & $\mathrm{O}(3 \mathrm{~B})-\mathrm{C}(8 \mathrm{~B})-\mathrm{C}(9 \mathrm{~B})$ & $110.11(16)$ \\
\hline$C(12 A)-C(2 A)-C(3 A)$ & $111.02(17)$ & $\mathrm{O}(3 \mathrm{~B})-\mathrm{C}(8 \mathrm{~B})-\mathrm{C}(7 \mathrm{~B})$ & $110.93(15)$ \\
\hline$C(2 A)-C(3 A)-C(4 A)$ & $110.00(17)$ & $C(9 B)-C(8 B)-C(7 B)$ & $114.99(15)$ \\
\hline$O(1 A)-C(4 A)-C(5 A)$ & $108.98(15)$ & $C(16 B)-C(9 B)-C(15 B)$ & $110.9(2)$ \\
\hline$O(1 A)-C(4 A)-C(3 A)$ & $109.63(14)$ & $C(16 \mathrm{~B})-\mathrm{C}(9 \mathrm{~B})-\mathrm{C}(8 \mathrm{~B})$ & $110.20(18)$ \\
\hline$C(5 A)-C(4 A)-C(3 A)$ & $109.46(17)$ & $C(15 B)-C(9 B)-C(8 B)$ & $112.38(18)$ \\
\hline$C(6 A)-C(5 A)-C(4 A)$ & 124.62(19) & $C(13 B)-C(12 B)-C(2 B)$ & $177.4(2)$ \\
\hline$C(5 A)-C(6 A)-C(7 A)$ & $125.36(19)$ & $C(1 \mathrm{~B})-\mathrm{O}(1 \mathrm{~B})-\mathrm{C}(4 \mathrm{~B})$ & $115.70(13)$ \\
\hline$C(6 A)-C(7 A)-C(14 A)$ & $111.95(17)$ & $\mathrm{C}(1 \mathrm{~B})-\mathrm{O}(2 \mathrm{~B})-\mathrm{C}(2 \mathrm{~B})$ & $114.26(13)$ \\
\hline
\end{tabular}


Table 4. Anisotropic displacement parameters $\left(\AA^{2} \times 10^{3}\right)$. The anisotropic displacement factor exponent takes the form: $-2 \pi^{2}\left[h^{2} a^{* 2} U^{11}+\ldots+2 h k a^{*} b^{*} U^{12}\right]$

\begin{tabular}{|c|c|c|c|c|c|c|}
\hline & $\mathrm{U}^{11}$ & $U^{22}$ & U33 & $U^{23}$ & $\mathrm{U}^{13}$ & $\mathrm{U}^{12}$ \\
\hline$C(1 A)$ & $43(1)$ & $38(1)$ & $33(1)$ & $3(1)$ & $-9(1)$ & $-14(1)$ \\
\hline$C(2 A)$ & $29(1)$ & $36(1)$ & $31(1)$ & $2(1)$ & $2(1)$ & $-2(1)$ \\
\hline$C(3 A)$ & $32(1)$ & $31(1)$ & $34(1)$ & $0(1)$ & $5(1)$ & $-3(1)$ \\
\hline$C(4 A)$ & 29(1) & $32(1)$ & $33(1)$ & $-3(1)$ & $3(1)$ & $-5(1)$ \\
\hline$C(5 A)$ & $34(1)$ & $32(1)$ & $32(1)$ & $-3(1)$ & $4(1)$ & $-6(1)$ \\
\hline$C(6 A)$ & $32(1)$ & $32(1)$ & $32(1)$ & $-1(1)$ & $2(1)$ & $-2(1)$ \\
\hline$C(7 A)$ & $32(1)$ & $33(1)$ & $29(1)$ & $-5(1)$ & $4(1)$ & $-5(1)$ \\
\hline$C(8 A)$ & $25(1)$ & $32(1)$ & $30(1)$ & $-1(1)$ & $3(1)$ & $-1(1)$ \\
\hline$C(9 A)$ & $30(1)$ & $33(1)$ & $39(1)$ & $-3(1)$ & $2(1)$ & $-2(1)$ \\
\hline$C(10 A)$ & $65(2)$ & $32(1)$ & $50(2)$ & $-3(1)$ & $-10(1)$ & $-2(1)$ \\
\hline$C(11 A)$ & $68(2)$ & $65(2)$ & $45(2)$ & $10(1)$ & $-3(1)$ & $-37(2)$ \\
\hline$C(12 A)$ & $29(1)$ & $45(1)$ & $40(1)$ & $-2(1)$ & $0(1)$ & $0(1)$ \\
\hline$C(13 A)$ & $39(1)$ & $61(2)$ & $53(2)$ & $5(1)$ & $-4(1)$ & $5(1)$ \\
\hline$C(14 A)$ & $54(2)$ & $41(1)$ & $33(1)$ & $2(1)$ & $-8(1)$ & $-10(1)$ \\
\hline$C(15 A)$ & $54(2)$ & $37(1)$ & $49(2)$ & $-8(1)$ & 12(1) & $1(1)$ \\
\hline$C(16 A)$ & $52(2)$ & $36(2)$ & $67(2)$ & $-2(1)$ & 19(1) & $-12(1)$ \\
\hline$O(1 A)$ & $40(1)$ & $38(1)$ & $30(1)$ & $3(1)$ & $0(1)$ & $-15(1)$ \\
\hline$O(2 A)$ & $32(1)$ & $43(1)$ & $36(1)$ & 1(1) & $0(1)$ & $-11(1)$ \\
\hline$O(3 A)$ & $31(1)$ & $47(1)$ & $41(1)$ & $-11(1)$ & $8(1)$ & $-4(1)$ \\
\hline$C(1 B)$ & $33(1)$ & $32(1)$ & $31(1)$ & $-2(1)$ & $-7(1)$ & $8(1)$ \\
\hline$C(2 B)$ & $26(1)$ & $33(1)$ & $33(1)$ & $-2(1)$ & $0(1)$ & $1(1)$ \\
\hline$C(3 B)$ & $29(1)$ & $31(1)$ & $36(1)$ & $0(1)$ & $2(1)$ & $5(1)$ \\
\hline$C(4 B)$ & $26(1)$ & $33(1)$ & $29(1)$ & $2(1)$ & $0(1)$ & $6(1)$ \\
\hline$C(5 B)$ & $33(1)$ & $35(1)$ & $30(1)$ & $4(1)$ & 1(1) & $7(1)$ \\
\hline$C(6 B)$ & $33(1)$ & $29(1)$ & $27(1)$ & $-1(1)$ & $0(1)$ & $3(1)$ \\
\hline$C(7 B)$ & $31(1)$ & $32(1)$ & $27(1)$ & $-2(1)$ & $-3(1)$ & $5(1)$ \\
\hline$C(8 B)$ & $27(1)$ & $33(1)$ & $26(1)$ & $2(1)$ & $-2(1)$ & $3(1)$ \\
\hline$C(9 B)$ & $42(1)$ & $30(1)$ & $33(1)$ & 1(1) & $-8(1)$ & $-1(1)$ \\
\hline$C(10 B)$ & $48(1)$ & $34(1)$ & $43(1)$ & $4(1)$ & $-2(1)$ & $-3(1)$ \\
\hline$C(11 B)$ & $49(1)$ & $53(2)$ & $41(1)$ & $-7(1)$ & $-4(1)$ & $20(1)$ \\
\hline$C(12 B)$ & $34(1)$ & $43(1)$ & $39(1)$ & $-2(1)$ & $-3(1)$ & $1(1)$ \\
\hline$C(13 B)$ & $41(1)$ & $61(2)$ & $61(2)$ & $-12(1)$ & $-3(1)$ & $-10(1)$ \\
\hline$C(14 B)$ & $60(2)$ & $33(1)$ & $46(2)$ & $-9(1)$ & $-21(1)$ & $5(1)$ \\
\hline$C(15 B)$ & $45(2)$ & $47(2)$ & $66(2)$ & $-2(1)$ & $-4(1)$ & $-14(1)$ \\
\hline$C(16 B)$ & $77(2)$ & $32(1)$ & $46(2)$ & $-6(1)$ & $-7(1)$ & $6(1)$ \\
\hline$O(1 B)$ & $33(1)$ & $35(1)$ & $29(1)$ & $-1(1)$ & $-4(1)$ & $9(1)$ \\
\hline $\mathrm{O}(2 \mathrm{~B})$ & $26(1)$ & $38(1)$ & $35(1)$ & $-3(1)$ & $-2(1)$ & $7(1)$ \\
\hline $\mathrm{O}(3 \mathrm{~B})$ & $39(1)$ & $43(1)$ & $32(1)$ & $-1(1)$ & $-7(1)$ & $12(1)$ \\
\hline
\end{tabular}


Table 5. Hydrogen coordinates $\left(\times 10^{4}\right)$ and isotropic displacement parameters $\left(\AA^{2} \times 10^{3}\right)$.

\begin{tabular}{|c|c|c|c|c|}
\hline & $x$ & $y$ & $z$ & $\mathrm{U}(\mathrm{eq})$ \\
\hline $\mathrm{H}(2 \mathrm{~A})$ & $1753(4)$ & $7020(30)$ & $7164(17)$ & $34(5)$ \\
\hline $\mathrm{H}(3 \mathrm{~A} 1)$ & 1563(5) & $4320(30)$ & 6218(17) & 44(6) \\
\hline $\mathrm{H}(3 \mathrm{~A} 2)$ & $1845(4)$ & $4460(30)$ & 5283(16) & $29(5)$ \\
\hline $\mathrm{H}(4 \mathrm{~A})$ & $1305(5)$ & $6890(30)$ & $5260(17)$ & $35(5)$ \\
\hline$H(5 A)$ & 1447(4) & 4390(30) & 3320(16) & $33(5)$ \\
\hline$H(6 A)$ & $825(4)$ & $5370(20)$ & 4156(16) & $24(4)$ \\
\hline $\mathrm{H}(7 \mathrm{~A})$ & $988(4)$ & 2730(30) & 2382(16) & $35(5)$ \\
\hline $\mathrm{H}(8 \mathrm{~A})$ & $843(4)$ & 1560(20) & 4311(16) & $28(5)$ \\
\hline $\mathrm{H}(9 \mathrm{~A})$ & $342(4)$ & $660(20)$ & 2380(16) & $28(5)$ \\
\hline $\mathrm{H}(10 \mathrm{~A})$ & 1495(5) & 9390(30) & 6270(20) & $59(7)$ \\
\hline $\mathrm{H}(10 \mathrm{~B})$ & $1514(6)$ & $10600(30)$ & $5100(20)$ & $67(8)$ \\
\hline $\mathrm{H}(10 \mathrm{C})$ & 1802(6) & 10710(30) & $6200(20)$ & $63(7)$ \\
\hline $\mathrm{H}(11 \mathrm{~A})$ & $2247(6)$ & $10380(30)$ & 4810(20) & $66(7)$ \\
\hline $\mathrm{H}(11 \mathrm{~B})$ & $2233(6)$ & $8610(40)$ & $3860(20)$ & 77(9) \\
\hline $\mathrm{H}(11 \mathrm{C})$ & 1975(6) & 10270(40) & $3680(20)$ & $80(9)$ \\
\hline $\mathrm{H}(13 \mathrm{~A})$ & $2478(6)$ & $3790(40)$ & 8610(20) & $79(9)$ \\
\hline $\mathrm{H}(14 \mathrm{~A})$ & 448(5) & 3190(30) & 1275(19) & $50(6)$ \\
\hline $\mathrm{H}(14 \mathrm{~B})$ & $361(5)$ & 4760(30) & 2223(19) & 47(6) \\
\hline $\mathrm{H}(14 \mathrm{C})$ & $645(5)$ & $5000(30)$ & 1283(19) & $56(7)$ \\
\hline $\mathrm{H}(15 \mathrm{~A})$ & $849(5)$ & 210(30) & 1530(20) & $50(6)$ \\
\hline $\mathrm{H}(15 \mathrm{~B})$ & $710(5)$ & $-1710(30)$ & 1870(20) & $62(7)$ \\
\hline$H(15 C)$ & 999(5) & $-880(30)$ & 2820(20) & $61(7)$ \\
\hline $\mathrm{H}(16 \mathrm{~A})$ & $328(6)$ & $-2070(40)$ & $3480(20)$ & $73(8)$ \\
\hline $\mathrm{H}(16 \mathrm{~B})$ & $244(6)$ & $-480(30)$ & $4360(20)$ & $63(7)$ \\
\hline $\mathrm{H}(16 \mathrm{C})$ & 596(6) & $-1330(30)$ & 4520(20) & 71(9) \\
\hline $\mathrm{H}(3 \mathrm{OA})$ & 251(6) & 2710(40) & $3890(20)$ & 65(9) \\
\hline $\mathrm{H}(2 \mathrm{~B})$ & $8318(4)$ & $7030(20)$ & $-1929(16)$ & $27(5)$ \\
\hline $\mathrm{H}(3 \mathrm{~B} 1)$ & $8298(4)$ & $4900(30)$ & $269(16)$ & $30(5)$ \\
\hline $\mathrm{H}(3 \mathrm{~B} 2)$ & $8555(4)$ & 4620(20) & $-751(15)$ & $24(5)$ \\
\hline $\mathrm{H}(4 \mathrm{~B})$ & $8813(4)$ & $7350(30)$ & $-197(16)$ & $30(5)$ \\
\hline$H(5 B)$ & $8735(5)$ & $5470(30)$ & 2131(18) & 45(6) \\
\hline $\mathrm{H}(6 \mathrm{~B})$ & $9313(4)$ & $6110(20)$ & $906(16)$ & $26(5)$ \\
\hline $\mathrm{H}(7 \mathrm{~B})$ & $9240(4)$ & 4520(30) & $3318(17)$ & $35(5)$ \\
\hline $\mathrm{H}(8 \mathrm{~B})$ & 9729(4) & $3610(20)$ & 1684(15) & 29(5) \\
\hline $\mathrm{H}(9 \mathrm{~B})$ & 9198(4) & $2430(30)$ & $907(17)$ & 40(5) \\
\hline $\mathrm{H}(10 \mathrm{D})$ & $8546(5)$ & $9510(30)$ & $-1430(18)$ & $44(6)$ \\
\hline $\mathrm{H}(10 \mathrm{E})$ & $8575(5)$ & 11050(30) & $-337(19)$ & $43(6)$ \\
\hline $\mathrm{H}(10 \mathrm{~F})$ & $8253(5)$ & 11060(30) & $-1340(20)$ & $60(7)$ \\
\hline$H(11 D)$ & $7851(5)$ & $10810(30)$ & 208(19) & $53(6)$ \\
\hline $\mathrm{H}(11 \mathrm{E})$ & $7906(5)$ & 9370(30) & $1300(20)$ & $58(7)$ \\
\hline $\mathrm{H}(11 \mathrm{~F})$ & $8165(5)$ & $11010(30)$ & 1300(20) & $53(6)$ \\
\hline
\end{tabular}




$\begin{array}{lrrrr}\mathrm{H}(13 \mathrm{~B}) & 7602(6) & 3640(30) & -2840(20) & 65(8) \\ \mathrm{H}(14 \mathrm{D}) & 9820(6) & 6520(40) & 2660(20) & 81(9) \\ \mathrm{H}(14 \mathrm{E}) & 9757(5) & 5680(30) & 4040(20) & 60(7) \\ \mathrm{H}(14 \mathrm{~F}) & 9527(5) & 7280(30) & 3503(19) & 57(7) \\ \mathrm{H}(15 \mathrm{D}) & 8955(5) & 220(30) & 2073(19) & 54(7) \\ \mathrm{H}(15 \mathrm{E}) & 8931(6) & 2030(40) & 2680(20) & 70(8) \\ \mathrm{H}(15 \mathrm{~F}) & 9215(6) & 570(30) & 3270(20) & 62(7) \\ \mathrm{H}(16 \mathrm{D}) & 9675(5) & -250(30) & 1810(20) & 52(6) \\ \mathrm{H}(16 \mathrm{E}) & 9401(6) & -530(40) & 720(20) & 59(7) \\ \mathrm{H}(16 \mathrm{~F}) & 9708(6) & 850(40) & 580(20) & 74(8) \\ \mathrm{H}(3 \mathrm{OB}) & 9663(5) & 2450(40) & 3900(20) & 53(8)\end{array}$

Table 6. Torsion angles $\left[^{\circ}\right]$.

\begin{tabular}{|c|c|}
\hline$O(2 A)-C(2 A)-C(3 A)-C(4 A)$ & $-54.7(2)$ \\
\hline$C(12 A)-C(2 A)-C(3 A)-C(4 A)$ & $-174.09(16)$ \\
\hline$C(2 A)-C(3 A)-C(4 A)-O(1 A)$ & $53.2(2)$ \\
\hline$C(2 A)-C(3 A)-C(4 A)-C(5 A)$ & 172.68(16) \\
\hline$O\left(1 A^{\prime}\right)-C\left(4 A^{\prime}\right)-C\left(5 A^{\prime}\right)-C\left(6 A^{\prime}\right)$ & $-127.3(2)$ \\
\hline$C(3 A)-C(4 A)-C(5 A)-C(6 A)$ & $112.8(2)$ \\
\hline$C(4 A)-C(5 A)-C(6 A)-C(7 A)$ & $-169.69(18)$ \\
\hline$C(5 A)-C(6 A)-C(7 A)-C(14 A)$ & $-120.7(2)$ \\
\hline$C(5 A)-C(6 A)-C(7 A)-C(8 A)$ & 114.4(2) \\
\hline$C(6 A)-C(7 A)-C(8 A)-O(3 A)$ & $62.60(19)$ \\
\hline$C(14 A)-C(7 A)-C(8 A)-O(3 A)$ & $-61.9(2)$ \\
\hline$C(6 A)-C(7 A)-C(8 A)-C(9 A)$ & $-170.95(16)$ \\
\hline$C(14 A)-C(7 A)-C(8 A)-C(9 A)$ & $64.6(2)$ \\
\hline$O(3 A)-C(8 A)-C(9 A)-C(16 A)$ & $-58.2(2)$ \\
\hline$C(7 A)-C(8 A)-C(9 A)-C(16 A)$ & $176.50(18)$ \\
\hline$O(3 A)-C(8 A)-C(9 A)-C(15 A)$ & $179.30(18)$ \\
\hline$C(7 A)-C(8 A)-C(9 A)-C(15 A)$ & $54.0(2)$ \\
\hline$O(2 A)-C(2 A)-C(12 A)-C(13 A)$ & $-139(3)$ \\
\hline$C(3 A)-C(2 A)-C(12 A)-C(13 A)$ & $-20(3)$ \\
\hline$C(5 A)-C(4 A)-O(1 A)-C(1 A)$ & $-173.53(16)$ \\
\hline$C(3 A)-C(4 A)-O(1 A)-C(1 A)$ & $-53.7(2)$ \\
\hline$O(2 A)-C(1 A)-O(1 A)-C(4 A)$ & $53.8(2)$ \\
\hline$C(11 A)-C(1 A)-O(1 A)-C(4 A)$ & $167.3(2)$ \\
\hline$C(10 A)-C(1 A)-O(1 A)-C(4 A)$ & $-70.1(2)$ \\
\hline$C(12 A)-C(2 A)-O(2 A)-C(1 A)$ & $177.94(16)$ \\
\hline$C(3 A)-C(2 A)-O(2 A)-C(1 A)$ & $57.0(2)$ \\
\hline$O(1 A)-C(1 A)-O(2 A)-C(2 A)$ & $-55.6(2)$ \\
\hline$C(11 A)-C(1 A)-O(2 A)-C(2 A)$ & $-169.28(19)$ \\
\hline$C(10 A)-C(1 A)-O(2 A)-C(2 A)$ & $68.2(2)$ \\
\hline $\mathrm{O}(2 \mathrm{~B})-\mathrm{C}(2 \mathrm{~B})-\mathrm{C}(3 \mathrm{~B})-\mathrm{C}(4 \mathrm{~B})$ & $-54.9(2)$ \\
\hline
\end{tabular}


$C(12 B)-C(2 B)-C(3 B)-C(4 B)$

$C(2 B)-C(3 B)-C(4 B)-O(1 B)$

$C(2 B)-C(3 B)-C(4 B)-C(5 B)$

$O(1 B)-C(4 B)-C(5 B)-C(6 B)$

$C(3 B)-C(4 B)-C(5 B)-C(6 B)$

$C(4 B)-C(5 B)-C(6 B)-C(7 B)$

$\mathrm{C}(5 \mathrm{~B})-\mathrm{C}(6 \mathrm{~B})-\mathrm{C}(7 \mathrm{~B})-\mathrm{C}(14 \mathrm{~B})$

$\mathrm{C}(5 \mathrm{~B})-\mathrm{C}(6 \mathrm{~B})-\mathrm{C}(7 \mathrm{~B})-\mathrm{C}(8 \mathrm{~B})$

$\mathrm{C}(6 \mathrm{~B})-\mathrm{C}(7 \mathrm{~B})-\mathrm{C}(8 \mathrm{~B})-\mathrm{O}(3 \mathrm{~B})$

$\mathrm{C}(14 \mathrm{~B})-\mathrm{C}(7 \mathrm{~B})-\mathrm{C}(8 \mathrm{~B})-\mathrm{O}(3 \mathrm{~B})$

$\mathrm{C}(6 \mathrm{~B})-\mathrm{C}(7 \mathrm{~B})-\mathrm{C}(8 \mathrm{~B})-\mathrm{C}(9 \mathrm{~B})$

$\mathrm{C}(14 \mathrm{~B})-\mathrm{C}(7 \mathrm{~B})-\mathrm{C}(8 \mathrm{~B})-\mathrm{C}(9 \mathrm{~B})$

$\mathrm{O}(3 \mathrm{~B})-\mathrm{C}(8 \mathrm{~B})-\mathrm{C}(9 \mathrm{~B})-\mathrm{C}(16 \mathrm{~B})$

$C(7 B)-C(8 B)-C(9 B)-C(16 B)$

$O(3 B)-C(8 B)-C(9 B)-C(15 B)$

$C(7 B)-C(8 B)-C(9 B)-C(15 B)$

$\mathrm{O}(2 \mathrm{~B})-\mathrm{C}(2 \mathrm{~B})-\mathrm{C}(12 \mathrm{~B})-\mathrm{C}(13 \mathrm{~B})$

$C(3 B)-C(2 B)-C(12 B)-C(13 B)$

$\mathrm{O}(2 \mathrm{~B})-\mathrm{C}(1 \mathrm{~B})-\mathrm{O}(1 \mathrm{~B})-\mathrm{C}(4 \mathrm{~B})$

$C(11 B)-C(1 B)-O(1 B)-C(4 B)$

$C(10 B)-C(1 B)-O(1 B)-C(4 B)$

$C(5 B)-C(4 B)-O(1 B)-C(1 B)$

$C(3 B)-C(4 B)-O(1 B)-C(1 B)$

$\mathrm{O}(1 \mathrm{~B})-\mathrm{C}(1 \mathrm{~B})-\mathrm{O}(2 \mathrm{~B})-\mathrm{C}(2 \mathrm{~B})$

$\mathrm{C}(11 \mathrm{~B})-\mathrm{C}(1 \mathrm{~B})-\mathrm{O}(2 \mathrm{~B})-\mathrm{C}(2 \mathrm{~B})$

$\mathrm{C}(10 \mathrm{~B})-\mathrm{C}(1 \mathrm{~B})-\mathrm{O}(2 \mathrm{~B})-\mathrm{C}(2 \mathrm{~B})$

$\mathrm{C}(12 \mathrm{~B})-\mathrm{C}(2 \mathrm{~B})-\mathrm{O}(2 \mathrm{~B})-\mathrm{C}(1 \mathrm{~B})$

$\mathrm{C}(3 \mathrm{~B})-\mathrm{C}(2 \mathrm{~B})-\mathrm{O}(2 \mathrm{~B})-\mathrm{C}(1 \mathrm{~B})$
$-174.78(16)$

$53.1(2)$

170.92(16)

$-128.1(2)$

112.5(2)

179.93(18)

$-118.3(2)$

119.3(2)

178.25(16)

$56.2(2)$

$-56.0(2)$

$-178.01(18)$

$-61.3(2)$

172.49(18)

$62.9(2)$

$-63.3(2)$

172(5)

$-67(5)$

54.48(19)

168.69(18)

$-69.7(2)$

$-175.53(15)$

$-54.4(2)$

$-55.55(19)$

$-169.77(17)$

68.7(2)

179.36(15)

57.3(2)

Table 7. Hydrogen bonds for 23 [ $\AA$ and $\left.{ }^{\circ}\right]$.

\begin{tabular}{|c|c|c|c|}
\hline $\bar{D}-\mathrm{H} \ldots \mathrm{A}$ & $\mathrm{d}(\mathrm{H} \ldots \mathrm{A})$ & $\mathrm{d}(\mathrm{D} \ldots \mathrm{A})$ & $<(\mathrm{DHA})$ \\
\hline $\mathrm{O}(3 \mathrm{~A})-\mathrm{H}(3 \mathrm{OA}) \ldots \mathrm{O}(3 \mathrm{~B}) \# 10.77(2)$ & $1.99(2)$ & $2.729(2)$ & 161(3) \\
\hline $\mathrm{O}(3 \mathrm{~B})-\mathrm{H}(3 \mathrm{OB}) \ldots \mathrm{O}(3 \mathrm{~A}) \# 20.78(2)$ & $2.01(2)$ & $2.735(2)$ & $156(2)$ \\
\hline
\end{tabular}

Symmetry transformations used to generate equivalent atoms: \#1 $x-1, y, z \quad \# 2-x+1, y$, $-z+1$ 
4-Methoxybenzyl ether (S)-28. 4-Methoxybenzyltrichloroacetimidate ${ }^{2}$ (freshly prepared from 4-methoxybenzyl alcohol, $6.3 \mathrm{~mL}, 51 \mathrm{mmol}$ ) was combined with alkynyl alcohol (S)-26 (5.6 g, $33.3 \mathrm{mmol}), \mathrm{CH}_{2} \mathrm{Cl}_{2}(10 \mathrm{~mL}$ ), and 10-camphorsulfonic acid (500 mg, 2.2 $\mathrm{mmol})$. The reaction was stirred for 4 days, diluted with water and extracted with ether. Organic fractions were dried with $\mathrm{MgSO}_{4}$, filtered, concentrated and chromatographed with pentane - ether (95:5 $\rightarrow$ 8: 2). The PMB ether $(S)-28$ was isolated in $74 \%$ yield (7.08 g).

${ }^{1} \mathrm{H}$ NMR $\left(\mathrm{CDCl}_{3}, 400 \mathrm{MHz}\right): \delta 7.30(\mathrm{~d}, 2 \mathrm{H}, J=8.4 \mathrm{~Hz}), 6.89(\mathrm{~d}, 2 \mathrm{H}, J=8.4 \mathrm{~Hz}), 5.88(\mathrm{~m}$, 1H), $5.12(\mathrm{~m}, 2 \mathrm{H}), 4.74(\mathrm{~d}, 1 \mathrm{H}, J=11.2 \mathrm{~Hz}), 4.48(\mathrm{~d}, 1 \mathrm{H}, J=11.6 \mathrm{~Hz}), 4.09(\mathrm{t}, 1 \mathrm{H}, J=6.4$ $\mathrm{Hz}), 3.82(\mathrm{~s}, 3 \mathrm{H}), 2.49(\mathrm{~m}, 2 \mathrm{H}), 0.22(\mathrm{~s}, 9 \mathrm{H}) .{ }^{13} \mathrm{C} \mathrm{NMR}\left(\mathrm{CDCl}_{3}, 100 \mathrm{MHz}\right): \delta 159.4$, 133.8, 130.1, 129.9, 117.7, 113.9, 104.4, 91.3, 70.2, 68.4, 55.4, 40.2, 0.13. IR (neat, $\mathrm{cm}^{-1}$ ): 2960 (s), 2900 (s), 2860 (s), 2835 (s), $2170(\mathrm{~m}), 1640(\mathrm{~m}), 1610(\mathrm{~s}), 1590(\mathrm{~m})$, 1510 (s), 1465 (m), 1330 (s), 1250 (s), 1170 (s), 1080 (s), 1040 (s), 845 (s), 760 (s). HRMS (FAB+): Calcd. for $\mathrm{C}_{17} \mathrm{H}_{24} \mathrm{O}_{2} \mathrm{Si}\left([\mathrm{M}]^{+}\right)$, 288.1546. Found, 288.1550. Anal. Calcd. for $\mathrm{C}_{17} \mathrm{H}_{24} \mathrm{O}_{2} \mathrm{Si}$ : C, 70.78; $\mathrm{H}$, 8.39. Found: $\mathrm{C}, 70.30 ; \mathrm{H}, 8.49$. [ $\left.\alpha\right]_{\mathrm{D}}-108$ (c $0.900, \mathrm{CHCl}_{3}$ ).

Epoxide 30. $\mathrm{m}$-Chloroperbenzoic acid $(12.9 \mathrm{~g}, 73.8 \mathrm{mmol})$ was added in one portion to a solution of PMB ether $(S)-28(7.08 \mathrm{~g}, 24.6 \mathrm{mmol})$ in $\mathrm{CH}_{2} \mathrm{Cl}_{2}(50 \mathrm{~mL})$. The reaction mixture was stirred at room temperature for $6 \mathrm{~h}$, then diluted with saturated aqueous $\mathrm{Na}_{2} \mathrm{SO}_{3}$ at $0^{\circ} \mathrm{C}$, stirred for 30 min, neutralized with saturated $\mathrm{NaHCO}_{3}$ and extracted with $\mathrm{CH}_{2} \mathrm{Cl}_{2}$ and ether. Organic fractions were dried with $\mathrm{MgSO}_{4}$, filtered, concentrated and chromatographed with pentane: ether (8: 2) to yield $435 \mathrm{mg} \mathrm{(6 \% )} \mathrm{of} \mathrm{starting} \mathrm{(S)-28}$ and $5.9 \mathrm{~g}(79 \%$ yield $)$ of the epoxide as an approximately $1: 1$ mixture of diastereomers. ${ }^{1} \mathrm{H}$ NMR $\left(\mathrm{CDCl}_{3}, 400 \mathrm{MHz}\right): \delta 7.29(\mathrm{~m}, 4 \mathrm{H}), 7.89(\mathrm{~m}, 4 \mathrm{H}), 4.75(\mathrm{~m}, 2 \mathrm{H}), 4.47(\mathrm{~m}, 2 \mathrm{H})$, $4.26(\mathrm{~m}, 2 \mathrm{H}), 3.81(\mathrm{~s}, 6 \mathrm{H}), 3.14(\mathrm{~m}, 2 \mathrm{H}), 2.79(\mathrm{~m}, 2 \mathrm{H}), 2.54(\mathrm{~m}, 2 \mathrm{H}), 1.95(\mathrm{~m}, 4 \mathrm{H}), 0.22$ (s, 9H), $0.21(\mathrm{~s}, 9 \mathrm{H}) \cdot{ }^{13} \mathrm{C} \mathrm{NMR}\left(\mathrm{CDCl}_{3}, 100 \mathrm{MHz}\right): \delta$ 159.5, 129.9, 129.1, 113.9, 104.1, 103.8, 91.9, 91.4, 70.5, 70.3, 66.4, 66.3, 55.4, 49.4, 49.3, 47.6, 47.2, 39.3, 39.9, 0.04 . IR (neat, $\left.\mathrm{cm}^{-1}\right)$ : $3000(\mathrm{~m}), 2960(\mathrm{~s}), 2865(\mathrm{~m}), 2170(\mathrm{~m}), 1725(\mathrm{~m}), 1615(\mathrm{~s}), 1585(\mathrm{~m})$, 1515 (s), 1465 (m), 1300 (s), 1250 (s), 1175 (s), 1075 (br, s), 1035 (s), 845 (br, s), 760 (s). $[\alpha]_{\mathrm{D}}-102\left(\mathrm{c} 0.865, \mathrm{CHCl}_{3}\right)$. 
A solution of this epoxide diastereomer mixture $(3.1 \mathrm{~g}, 10.2 \mathrm{mmol})$ and $(\mathrm{S}, \mathrm{S})$-salen Co(III) OAc polymer-supported cobalt catalyst ${ }^{4}(500 \mathrm{mg})$ in THF $(8 \mathrm{~mL})$ was stirred at room temperature for $5 \mathrm{~min}$, and then distilled water $(750 \mu \mathrm{L}, 41.7 \mathrm{mmol})$ was added. After stirring for $22 \mathrm{~h}$, the reaction was poured into a separatory funnel, and the layers were separated. The organic layer was dried with $\mathrm{Na}_{2} \mathrm{SO}_{4}$, filtered, concentrated. After chromatography of the residue with pentane - ether $(8: 2), 1.3 \mathrm{~g}$ (42\% yield) of the epoxide $\mathbf{3 0}$ was obtained as a single diastereomer. Continued chromatography with hexane - ethyl acetate ( $1: 1)$ yielded $1.78 \mathrm{~g}$ ( $54 \%$ yield) of the diol 32 as a $4: 1$ mixture of diastereomers.

Epoxide 30: ${ }^{1} \mathrm{H}$ NMR $\left(\mathrm{CDCl}_{3}, 400 \mathrm{MHz}\right): \delta 7.30(\mathrm{~d}, 2 \mathrm{H}, \mathrm{J}=8.4 \mathrm{~Hz}), 6.89(\mathrm{~d}, 2 \mathrm{H}, J=8.4$ $\mathrm{Hz}), 4.76(\mathrm{~d}, 1 \mathrm{H}, J=11.2 \mathrm{~Hz}), 4.48(\mathrm{~d}, 1 \mathrm{H}, J=11.2 \mathrm{~Hz}), 4.27(\mathrm{~m}, 1 \mathrm{H}), 3.81(\mathrm{~s}, 3 \mathrm{H}), 3.15$ $(\mathrm{m}, 1 \mathrm{H}), 2.81(\mathrm{~m}, 1 \mathrm{H}), 2.55(\mathrm{~m}, 1 \mathrm{H}), 1.98(\mathrm{~m}, 1 \mathrm{H}), 1.91(\mathrm{~m}, 1 \mathrm{H}), 0.21(\mathrm{~s}, 9 \mathrm{H}) .{ }^{13} \mathrm{C} N M R$ $\left(\mathrm{CDCl}_{3}, 100 \mathrm{MHz}\right): \delta 159.5,130.0,129.8,114.0,104.0,91.5,70.5,66.3,55.4,49.3$, 47.6, 39.3, 0.1. IR (neat, $\left.\mathrm{cm}^{-1}\right): 3000(\mathrm{~m}), 2960(\mathrm{~s}), 2865(\mathrm{~m}), 2170(\mathrm{~m}), 1720(\mathrm{~m}), 1610$ (s), $1585(\mathrm{~m}), 1515(\mathrm{~s}), 1465(\mathrm{~m}), 1300(\mathrm{~m}), 1250$ (s), $1175(\mathrm{~m}), 1090$ (s, br), 1035 (s), 845 (s, br), 760 (s). HRMS (FAB+): Calcd. for $\mathrm{C}_{17} \mathrm{H}_{24} \mathrm{O}_{3} \mathrm{SiLi}\left([\mathrm{M}+\mathrm{Li}]^{+}\right)$) 311.1655. Found, 311.1669. Anal. Calcd. for $\mathrm{C}_{17} \mathrm{H}_{24} \mathrm{O}_{3} \mathrm{Si}$ : C, 67.07; H, 7.95. Found: C, 66.91; $\mathrm{H}, 8.01$. $[\alpha]_{D}$ -94 (c $\left.0.795, \mathrm{CHCl}_{3}\right)$.

Diol 32: ${ }^{1} \mathrm{H}$ NMR $\left(\mathrm{CDCl}_{3}, 400 \mathrm{MHz}\right): \delta 7.21(\mathrm{~d}, 2 \mathrm{H}, J=8.4 \mathrm{~Hz}), 6.83(\mathrm{~d}, 2 \mathrm{H}, J=8.8 \mathrm{~Hz})$, $4.70(\mathrm{~d}, 1 \mathrm{H}, J=11.2 \mathrm{~Hz}), 4.40(\mathrm{~d}, 1 \mathrm{H}, J=11.2 \mathrm{~Hz}), 4.24(\mathrm{~m}, 1 \mathrm{H}), 3.86(\mathrm{~m}, 1 \mathrm{H}), 3.75(\mathrm{~s}$, $3 \mathrm{H}), 3.52(\mathrm{~m}, 1 \mathrm{H}), 3.42(\mathrm{~m}, 1 \mathrm{H}), 3.30(\mathrm{~s}, \mathrm{br}, 1 \mathrm{H},-\mathrm{OH}), 2.51(\mathrm{~s}, \mathrm{br}, 1 \mathrm{H},-\mathrm{OH}), 1.91(\mathrm{~m}$, $1 \mathrm{H}), 1.80(\mathrm{~m}, 1 \mathrm{H}), 0.15(\mathrm{~s}, 9 \mathrm{H}) .{ }^{13} \mathrm{C} \mathrm{NMR}\left(\mathrm{CDCl}_{3}, 100 \mathrm{MHz}\right): \delta 159.6,130.0,129.4$, 114.0, 103.6, 91.9, 70.8, 70.5, 67.65, 66.54, 55.4, 39.0, 0.0. IR $\left(\mathrm{KBr}, \mathrm{cm}^{-1}\right): 3200(\mathrm{br}$, m), $2955(\mathrm{~m}), 2850(\mathrm{~m}), 2170(\mathrm{w}), 1615(\mathrm{~m}), 1515(\mathrm{~s}), 1405(\mathrm{~m}), 1250(\mathrm{~s}), 1175(\mathrm{~m})$, 1095 (s), 1025 (s), 890 (m), 840 (s), 760 (m). HRMS (FAB+): Calcd. for $\mathrm{C}_{17} \mathrm{H}_{26} \mathrm{O}_{4} \mathrm{SiLi}$ $\left([\mathrm{M}+\mathrm{Li}]^{+}\right)$, 329.1760. Found, 329.1774. Anal. Calcd. for $\mathrm{C}_{17} \mathrm{H}_{26} \mathrm{O}_{4} \mathrm{Si}$ : C, 63.32; $\mathrm{H}, 8.13$. Found: C, 63.34; $\mathrm{H}, 8.15$. $[\alpha]_{\mathrm{D}}-114$ (c $\left.0.860, \mathrm{CHCl}_{3}, \mathrm{dr}, 4: 1\right)$.

4. Annis, D. A.; Jacobsen, E. N. J. Am. Chem. Soc. 1999, 121, 4147. 
(R)-Epoxide 33. A suspension of $\mathrm{CuBr}(1.56 \mathrm{~g}, 10.8 \mathrm{mmol})$ in $\mathrm{Et}_{2} \mathrm{O}(100 \mathrm{~mL})$ was cooled to $-70^{\circ} \mathrm{C}$, and $1 \mathrm{M} \mathrm{THF}$ solution of vinylmagnesium bromide $(52 \mathrm{~mL}, 52.0 \mathrm{mmol})$, followed by addition of $(R)-(-)$-epichlorohydrin $(5 \mathrm{~g}, 54.0 \mathrm{mmol})$. The reaction was slowly warmed to $-40^{\circ} \mathrm{C}$ and stirred for 2 days, then poured into water, filtered through Celite and extracted with ether. Organic fractions were dried with $\mathrm{MgSO}_{4}$ and filtered. Solvent was distilled off at normal pressure and the residue was distilled in vacuum producing corresponding chlorohydrin in $84 \%$ yield $\left(5.47 \mathrm{~g}\right.$, bp $55-59^{\circ} \mathrm{C} / 15$ torr). ${ }^{1} \mathrm{H}$ and ${ }^{13} \mathrm{C}$ NMR were identical to corresponding racemic compound ${ }^{5},[\alpha]^{\mathrm{RT}}-5$ (c 1.400, $\left.\mathrm{CHCl}_{3}\right)$.

$\mathrm{KOH}(2.9 \mathrm{~g})$ was combined with freshly prepared chlorohydrin $(5.4 \mathrm{~g}, 44.8 \mathrm{mmol})$ in a reaction flask fitted with a distillation head. Wet epoxide $\mathbf{3} 3$ was distilled at atmospheric pressure (bp $80-85^{\circ} \mathrm{C}$ ), then dried with $\mathrm{MgSO}_{4}$ and decanted producing clean $33(3.0 \mathrm{~g}$, $81 \%$ yield). ${ }^{1} \mathrm{H}$ and ${ }^{13} \mathrm{C}$ NMR were identical to the corresponding racemic compound ${ }^{5}$, $\left.[\alpha]^{\mathrm{RT}} 0\left(\mathrm{c} 1.575, \mathrm{CHCl}_{3}\right)\right)$.

(4R)-7-(trimethylsilyl)-1-hepten-6-yne-4-ol (34). A solution of (trimethylsilyl)acetylene $(10.3 \mathrm{~mL}, 71 \mathrm{mmol})$ in THF $(220 \mathrm{~mL})$ was cooled to $-78^{\circ} \mathrm{C}$, and $2.5 \mathrm{M}$ THF solution of $n$ BuLi (30 mL, $75 \mathrm{mmol}$ ) was added. After addition, the reaction was warmed to ambient temperature and stirred for $1.5 \mathrm{~h}$, then $\mathrm{BF}_{3}-\mathrm{Et}_{2} \mathrm{O}(9.3 \mathrm{~mL}, 78.5 \mathrm{mmol})$ was added at $-78^{\circ} \mathrm{C}$ producing the alkynylboron nucleophile. After $20 \mathrm{~min}$, a solution of epoxide 33 $(3.0 \mathrm{~g}, 35.7 \mathrm{mmol})$ was added via cannula to the reaction. The mixture was stirred at $-78^{\circ} \mathrm{C}$ for $1 \mathrm{~h}$, then diluted with saturated aqueous $\mathrm{NaCl}$ and extracted with ether. Organic fractions were dried with $\mathrm{MgSO}_{4}$, filtered and concentrated. The residue was vacuum distilled to yield a fraction with bp. ca. $160^{\circ} \mathrm{C} / 0.4$ torr, which was additionally purified by flash chromatography with pentane - ether $(85: 15)$ yielding $4.89 \mathrm{~g}(75 \%)$ of alcohol 34.

${ }^{1} \mathrm{H}$ NMR $\left(\mathrm{CDCl}_{3}, 600 \mathrm{MHz}\right): \delta 5.84(\mathrm{~m}, 1 \mathrm{H}), 5.15(\mathrm{~m}, 2 \mathrm{H}), 3.81(\mathrm{~m}, 1 \mathrm{H}), 2.44(\mathrm{~m}, 2 \mathrm{H})$, $2.38(\mathrm{~m}, 1 \mathrm{H}), 2.30(\mathrm{~m}, 1 \mathrm{H}), 0.17(\mathrm{~s}, 9 \mathrm{H}) .{ }^{13} \mathrm{C} \mathrm{NMR}\left(\mathrm{CDCl}_{3}, 150 \mathrm{MHz}\right): \delta$ 134.3, 118.5, 103.1, 87.9, 69.2, 40.8, 28.2, 0.2. IR (neat, $\left.\mathrm{cm}^{-1}\right): 3400(\mathrm{~m}, \mathrm{br}), 2960(\mathrm{~m}), 2915(\mathrm{~m})$, $2175(\mathrm{~m}), 1640(\mathrm{w}), 1420(\mathrm{w}, \mathrm{br}), 1250(\mathrm{~m}), 1080(\mathrm{~m}), 1020(\mathrm{~m}), 920(\mathrm{~m}), 840(\mathrm{~s}), 760$ (m). HRMS (FAB+): Calcd. for $\mathrm{C}_{10} \mathrm{H}_{18} \mathrm{OSiLi}\left([\mathrm{M}+\mathrm{Li}]^{+}\right)$, 189.1287. Found, 189.1281. Anal.

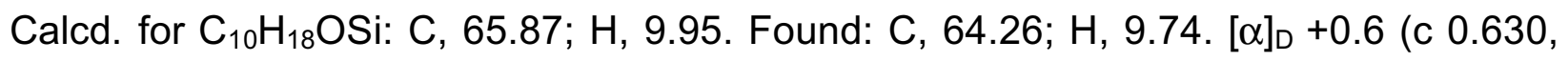
$\left.\mathrm{CHCl}_{3}\right)$.

5. (a) Schuda, A.; Mazzocchi, P. H.; Fritz, G.; Morgan, T. Synthesis 1986, 309. (b) Zemribo, R.; 


\section{Preparation of 35:}

Epoxidation of hydroxyalkene 34: Epoxidation of 34 was performed in analogy to epoxidation of $(S)$-28. The above epoxyalcohol was obtained in $77 \%$ yield with $\mathrm{dr}, 1.2: 1$ : ${ }^{1} \mathrm{H} \mathrm{NMR}\left(\mathrm{CDCl}_{3}, 600 \mathrm{MHz}\right): \delta 4.00(\mathrm{~m}, 2.2 \mathrm{H}), 3.16(\mathrm{~m}, 1 \mathrm{H}), 3.11(\mathrm{~m}, 1.2 \mathrm{H}), 2.84(\mathrm{t}, 1 \mathrm{H}$, $J=4.8 \mathrm{~Hz}), 2.80(\mathrm{t}, 1.2 \mathrm{H}, J=4.8 \mathrm{~Hz}), 2.60(\mathrm{~m}, 1.2 \mathrm{H}), 2.54(\mathrm{~m}, 1 \mathrm{H}), 2.49(\mathrm{~m}, 3.4 \mathrm{H}), 2.44$ $(\mathrm{m}, 1 \mathrm{H}), 2.41$ (s, br, $2.2 \mathrm{H},-\mathrm{OH}), 0.16(\mathrm{~s}, 19.8 \mathrm{H}) .{ }^{13} \mathrm{C} \mathrm{NMR}\left(\mathrm{CDCl}_{3}, 150 \mathrm{MHz}\right): \delta 102.7$, 88.1, 68.6, 67.8, 50.3, 50.0, 47.1, 46.8, 38.7, 38.4, 29.1, 28.9, 0.21.

Protection of the C4-alcohol: The epoxyalcohol $(1.68 \mathrm{~g}, 8.5 \mathrm{mmol})$ from the previous experiment was dissolved in DMF (14 mL), and $\mathrm{NaH}(60 \%$ in mineral oil, $510 \mathrm{mg}, 12.7$ $\mathrm{mmol}$ ) was added in one portion (Caution! Gas evolution!). The reaction was stirred for $10 \mathrm{~min}$ and 4-methoxybenzylchloride $(3.45 \mathrm{~mL}, 25.5 \mathrm{mmol})$ was slowly added at $0^{\circ} \mathrm{C}$, followed by addition of crystalline $\mathrm{Bu}_{4} \mathrm{NI}(4.7 \mathrm{~g}, 12.7 \mathrm{mmol})$. The mixture was warmed to ambient temperature and stirred for $25 \mathrm{~h}$, then diluted with water and extracted with ether. Combined organic fractions were dried with $\mathrm{MgSO}_{4}$, filtered, concentrated and chromatographed with pentane - ether $(7: 3)$ producing the PMB-protected epoxyalcohol in $73 \%$ yield $(1.54 \mathrm{~g}$, dr, 1.2: 1), with loss of the alkynyl TMS substituent. ${ }^{1} \mathrm{H}$ NMR $\left(\mathrm{CDCl}_{3}, 600 \mathrm{MHz}\right): \delta 7.29(\mathrm{~m}, 4.4 \mathrm{H}), 6.89(\mathrm{~m}, 4.4 \mathrm{H}), 4.62(\mathrm{~m}, 2.2 \mathrm{H}), 4.49(\mathrm{~m}$, 2.2H), $3.81(\mathrm{~m}, 6.6 \mathrm{H}), 3.73(\mathrm{~m}, 2.2 \mathrm{H}), 3.09(\mathrm{~m}, 1 \mathrm{H}), 3.06(\mathrm{~m}, 1.2 \mathrm{H}), 2.81(\mathrm{t}, 1 \mathrm{H}, J=4.2$ $\mathrm{Hz}), 2.74(\mathrm{t}, 1.2 \mathrm{H}, J=4.2 \mathrm{~Hz}), 2.58-2.48(\mathrm{~m}, 6.6 \mathrm{H}), 2.03(\mathrm{~m}, 2.2 \mathrm{H}), 1.99-1.89(\mathrm{~m}, 4.4 \mathrm{H})$. ${ }^{13} \mathrm{C} \mathrm{NMR}\left(\mathrm{CDCl}_{3}, 150 \mathrm{MHz}\right): \delta 159.4(2 \mathrm{C}), 130.35,130.32,129.59,129.54,114.0(2 \mathrm{C})$, $80.80,80.71,74.83,74.75,71.6,71.1,70.6(2 \mathrm{C}), 55.4(2 \mathrm{C}), 49.60,49.56,47.7,46.8$, 37.6, 36.8, 24.3, 23.9. IR (neat, $\mathrm{cm}^{-1}$ ): $3290(\mathrm{~m}), 2995(\mathrm{~m}), 2915(\mathrm{~m}), 2870(\mathrm{~m}), 1610$ (m), 1510 (s), $1465(\mathrm{~m}), 1350(\mathrm{~m}), 1300$ (m), 1250 (s), 1175 (m), 1095 (s), 1035 (s), 820 (s). HRMS (FAB+): Calcd. for $\mathrm{C}_{15} \mathrm{H}_{18} \mathrm{O}_{3} \mathrm{SiLi}\left([\mathrm{M}+\mathrm{Li}]^{+}\right)$, 253.1416. Found, 253.1422. Anal. Calcd. for $\mathrm{C}_{15} \mathrm{H}_{18} \mathrm{O}_{3}$ : C, 73.15; $\mathrm{H}, 7.37$. Found: $\mathrm{C}, 72.90 ; \mathrm{H}, 7.42$. $[\alpha]_{D}+46$ (c 0.690 , $\mathrm{CHCl}_{3}$ ).

Replacement of the alkynyl-TMS substituent: PMB-protected epoxyalcohol (610 mg, $2.48 \mathrm{mmol}$ ) from previous experiment was dissolved in THF $(15 \mathrm{~mL})$. To this solution, 2.5M THF solution of $n$-BuLi $(1.1 \mathrm{~mL}, 2.75 \mathrm{mmol})$ was added at $-55^{\circ} \mathrm{C}$. The reaction

Mead, K. T. Tetrahedron Lett. 1998, 39, 3895. 
was stirred for 10 min at $-50^{\circ} \mathrm{C}$ and $\mathrm{TMSCl}$ was slowly added. The mixture was stirred for $0.5 \mathrm{~h}$ at $-40^{\circ} \mathrm{C}$, diluted with saturated aqueous $\mathrm{NaCl}$ and extracted with ether. The organic fractions were dried with $\mathrm{MgSO}_{4}$, filtered and concentrated. The residue was chromatographed with pentane - ether ( $7: 3$ ) producing $(4 R)-35$ in 55\% yield (434 mg, dr, 1.2: 1).

${ }^{1} \mathrm{H}$ NMR $\left(\mathrm{CDCl}_{3}, 600 \mathrm{MHz}\right): \delta 7.30(\mathrm{~d}, 4.4 \mathrm{H}, J=8.4 \mathrm{~Hz}), 6.89(\mathrm{~d}, 4.4 \mathrm{H}, J=8.4 \mathrm{~Hz}), 4.65$ $(\mathrm{d}, 1 \mathrm{H}, J=11.4 \mathrm{~Hz}), 4.62(\mathrm{~d}, 1.2 \mathrm{H}, J=11.4 \mathrm{~Hz}), 4.52(\mathrm{~d}, 1 \mathrm{H}, J=11.4 \mathrm{~Hz}), 4.49(\mathrm{~d}, 1.2 \mathrm{H}$, $J=11.4 \mathrm{~Hz}), 3.81(\mathrm{~s}, \mathrm{br}, 6.6 \mathrm{H}), 3.10(\mathrm{~m}, 1 \mathrm{H}), 3.06(\mathrm{~m}, 1.2 \mathrm{H}), 2.81(\mathrm{t}, 1 \mathrm{H}, J=4.2 \mathrm{~Hz})$, $2.74(\mathrm{t}, 1.2 \mathrm{H}, \mathrm{J}=4.2 \mathrm{~Hz}), 2.65-2.57(\mathrm{~m}, 2.2 \mathrm{H}), 2.55-2.48(\mathrm{~m}, 6.6 \mathrm{H}), 1.96-1.75(\mathrm{~m}, 4.4 \mathrm{H})$, $0.16(\mathrm{~s}, 19.8 \mathrm{H}) .{ }^{13} \mathrm{C} \mathrm{NMR}\left(\mathrm{CDCl}_{3}, 150 \mathrm{MHz}\right): \delta 159.4(2 \mathrm{C}), 130.52,130.47,129.58$, 129.54, $114.0(2 \mathrm{C}), 103.56,103.51,87.0(2 \mathrm{C}), 75.2(2 \mathrm{C}), 71.7(2 \mathrm{C}), 71.2(2 \mathrm{C}), 55.5$ (2C), 49.7, 49.6, 47.8, 47.0, 37.7, 37.2, 25.9, 25.6, 0.2. IR (neat, $\left.\mathrm{cm}^{-1}\right): 2995(\mathrm{~m}), 2955$ (s), 2915 (s), 2860 (m), 2175 (s), 1610 (s), 1510 (s), 1465 (m), $1345(\mathrm{~m}), 1300(\mathrm{~m})$, 1250 (s), 1175 (s), 1095 (s), 1035 (s), 845 (s), 760 (s). [ $\alpha]_{\mathrm{D}}+26$ (c 0.195, $\mathrm{CHCl}_{3}$ ).

Hydrolytic kinetic resolution of epoxide (4R)-35 with Jacobsen cobalt catalyst. A solution of epoxide (4R)-35 (765 mg, $2.4 \mathrm{mmol}$ ) and (S, S)-salenCo(III)OAc (160 mg, $0.24 \mathrm{mmol})$ in THF $(1 \mathrm{~mL})$ was stirred at room temperature for $5 \mathrm{~min}$, and then distilled water $(200 \mu \mathrm{L}, 11.1 \mathrm{mmol})$ was added. After stirring for $1 \mathrm{~h}$, the reaction was dried with $\mathrm{Na}_{2} \mathrm{SO}_{4}$, filtered, concentrated. After chromatography of the residue with pentane- ether (85: 15), $247 \mathrm{mg}$ ( $32 \%$ yield) of epoxide (2S, $4 R$ )-36 was obtained as a single

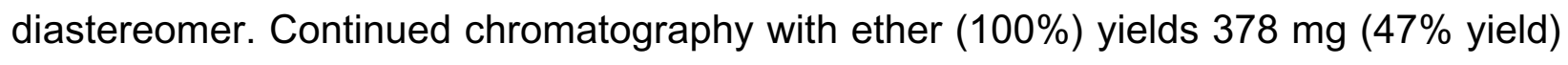
of diol $(2 R, 4 R)-37$ as a single diastereomer.

(2S, 4R)-36. ${ }^{1} \mathrm{H}$ NMR $\left(\mathrm{CDCl}_{3}, 600 \mathrm{MHz}\right): \delta 7.29(\mathrm{~d}, 2 \mathrm{H}, \mathrm{J}=9.0 \mathrm{~Hz}), 6.88(\mathrm{~d}, 2 \mathrm{H}, J=9.0$ Hz), $4.62(\mathrm{~d}, 1 \mathrm{H}, J=11.4 \mathrm{~Hz}), 4.49(\mathrm{~d}, 1 \mathrm{H}, J=11.4 \mathrm{~Hz}), 3.81(\mathrm{~s}, 3 \mathrm{H}), 3.06(\mathrm{~m}, 1 \mathrm{H}), 2.74$ (t, $1 \mathrm{H}, J=4.8 \mathrm{~Hz}), 2.63(\mathrm{dd}, 1 \mathrm{H}, J=17.4,6.0 \mathrm{~Hz}), 2.49(\mathrm{~m}, 3 \mathrm{H}), 1.92(\mathrm{~m}, 1 \mathrm{H}), 1.85(\mathrm{~m}$, $1 \mathrm{H}), 0.16(\mathrm{~s}, 9 \mathrm{H}) .{ }^{13} \mathrm{C} \mathrm{NMR}\left(\mathrm{CDCl}_{3}, 400 \mathrm{MHz}\right): \delta 159.4,130.5,129.5,113.9,103.5,87.0$, 75.2, 71.1, 55.5, 49.7, 47.0, 37.2, 25.6, 0.2. IR (neat, $\left.\mathrm{cm}^{-1}\right): 2955(\mathrm{~m}), 2916(\mathrm{~s}), 2850$ (m), $2175(\mathrm{~m}), 1610(\mathrm{~m}), 1515(\mathrm{~s}), 1465(\mathrm{~m}), 1250(\mathrm{~s}), 1095$ (m, br), 1035 (m), 845 (s, br), $760(\mathrm{~m})$. HRMS (FAB+): Calcd. for $\mathrm{C}_{18} \mathrm{H}_{26} \mathrm{O}_{3} \mathrm{SiLi}\left([\mathrm{M}+\mathrm{Li}]^{+}\right), 325.1811$. Found, 325.1823. $[\alpha]_{\mathrm{D}}+24\left(\mathrm{c} 0.155, \mathrm{CHCl}_{3}\right)$. 


\section{Attempted hydration from iodocyclization of the tert-butyl carbonate derivative of}

38: In our original approach ${ }^{6}$ we could selectively convert the internal alkyne of diyne 7 into hydroxyketone 8 (figure 4 of main text) through iodocyclization of a carbonate derivative of the homoproparylic alcohol, followed by deiodination under radical conditions. Therefore, the alkynyl alcohol $\mathbf{3 8}$ was converted to the corresponding tertbutylcarbonate ester followed by cyclization with $\mathrm{IBr}$ to cleanly produce light-sensitive cyclic iodocarbonate $\mathbf{i}$. However radical deiodination of $\mathbf{i}$ with $\mathrm{Bu}_{3} \mathrm{SnH}$ did not produce desired enol carbonate, but instead resulted in loss of olefin at C28-C29 and loss of $p$ methoxybenzyl ether to give a compound tentatively assigned as structure ii (scheme i). Indeed, generation of free radical iii at C24 five atoms away from the C28-C29 unsaturation most likely resulted in radical cyclization to produce a five-membered ring. The newly formed radical at C29 can then abstract a benzylic hydrogen from the PMB ether, resulting in deprotection of the C31-alcohol via proposed intermediate iv.

Scheme i. Radical cyclization side-reaction upon deiodination

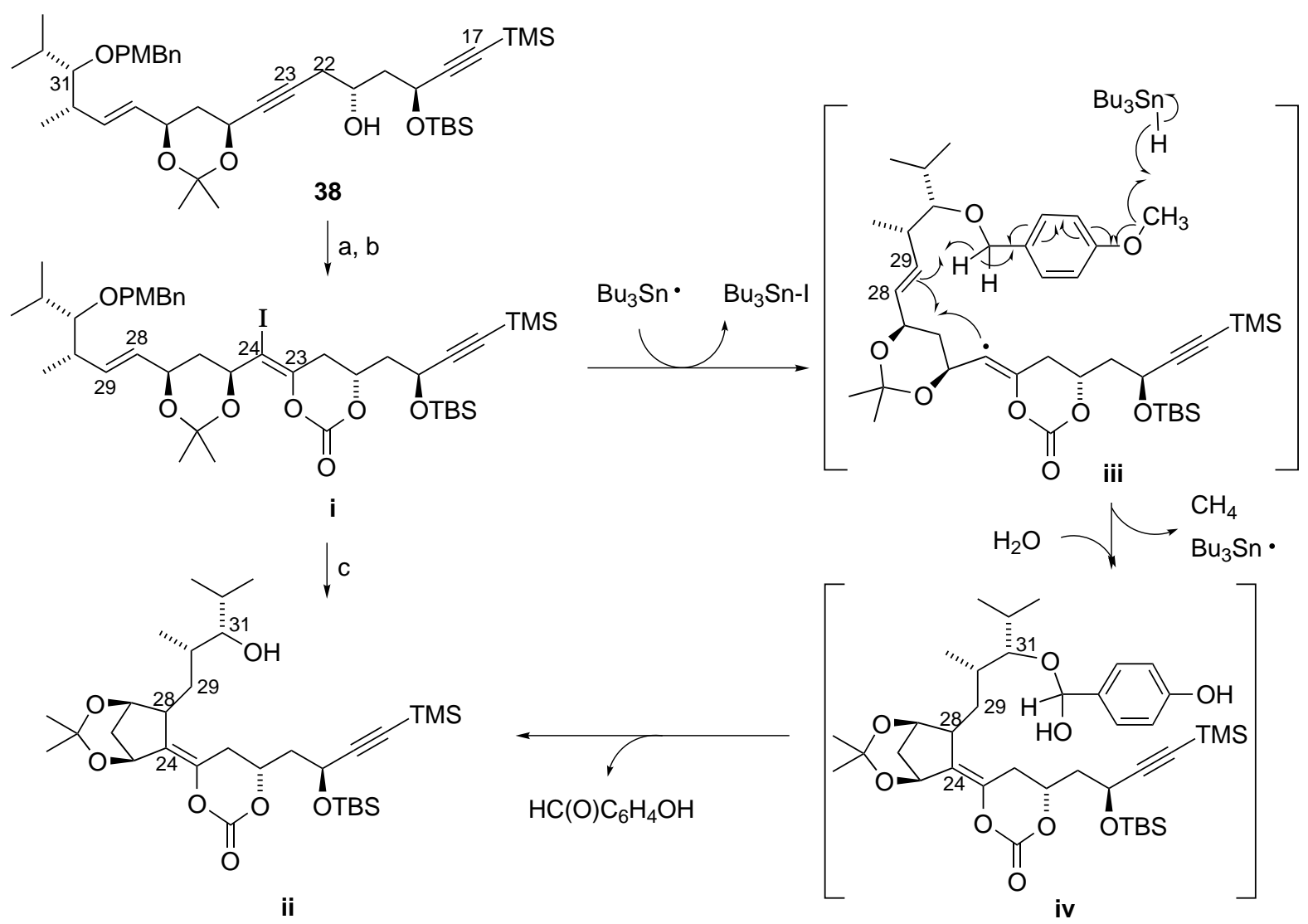

(a) $\mathrm{Boc}_{2} \mathrm{O}, \mathrm{Et}_{3} \mathrm{~N}$, cat. DMAP, $\mathrm{CH}_{2} \mathrm{Cl}_{2}(77 \%)$. (b) $\mathrm{IBr}, \mathrm{CH}_{2} \mathrm{Cl}_{2} /$ toluene, $0^{\circ} \mathrm{C}$. (c) $\mathrm{Bu}_{3} \mathrm{SnH}$, cat. $\mathrm{Et}_{3} \mathrm{~N}$, hexane, $0^{\circ}$ to $20^{\circ} \mathrm{C}$

6. Burova, S. A.; McDonald, F. E. J. Am. Chem. Soc. 2002, 124, 8188. 
Compound ii was partially characterized by ${ }^{1} \mathrm{H}$ NMR spectroscopy: $\left(\mathrm{CDCl}_{3}, 600 \mathrm{MHz}\right): \delta$ $4.81(\mathrm{~m}, 1 \mathrm{H}), 4.67(\mathrm{dm}, 1 \mathrm{H}, J=11.4 \mathrm{~Hz}), 4.46(\mathrm{dd}, 1 \mathrm{H}, J=10.8,3.6 \mathrm{~Hz}), 3.89(\mathrm{~m}, 1 \mathrm{H})$, $3.26(\mathrm{dd}, 1 \mathrm{H}, J=9.0,5.4 \mathrm{~Hz}), 2.60(\mathrm{dd}, 2 \mathrm{H}, J=5.4,1.8 \mathrm{~Hz}), 2.23(\mathrm{~m}, 1 \mathrm{H}), 2.10-2.01(\mathrm{~m}$, $3 \mathrm{H}), 1.72(\mathrm{~m}, 2 \mathrm{H}), 1.47(\mathrm{~s}, 6 \mathrm{H}), 1.00(\mathrm{~d}, 3 \mathrm{H}, \mathrm{J}=6.0 \mathrm{~Hz}), 0.93(\mathrm{~m}, 6 \mathrm{H}), 0.91(\mathrm{~s}, 9 \mathrm{H}), 0.16$ $(\mathrm{s}, 9 \mathrm{H}), 0.14(\mathrm{~s}, 3 \mathrm{H}), 0.09(\mathrm{~s}, 3 \mathrm{H})$.

Alkynyl alcohol 39. A solution of alkyne $23(288 \mathrm{mg}, 0.75 \mathrm{mmol})$ in THF (5 mL) was cooled to $-50^{\circ} \mathrm{C}$, and a solution of $n$-BuLi $(0.33 \mathrm{~mL}, 0.82 \mathrm{mmol})$ in hexane was added. The reaction was stirred $2.5 \mathrm{~h}$ at -60 to $-40^{\circ} \mathrm{C}$ and $\mathrm{BF}_{3} \mathrm{OEt}_{2}(100 \mu \mathrm{L}, 0.86 \mathrm{mmol})$ was added at $-78^{\circ} \mathrm{C}$. The mixture was stirred $15 \mathrm{~min}$ at $-78^{\circ} \mathrm{C}$ and a solution of epoxide 30 (520 mg, $1.7 \mathrm{mmol})$ in THF ( $2 \mathrm{~mL}$ ) was added via cannula. The mixture was stirred at $-78^{\circ} \mathrm{C}$ for $1.5 \mathrm{~h}$, then diluted with saturated aqueous $\mathrm{NaCl}$ and extracted with ether. Organic fractions were dried with $\mathrm{MgSO}_{4}$, filtered and concentrated. After chromatography of the residue with pentane - ether (6: 4) $328 \mathrm{mg}$ of a mixture of starting epoxide $\mathbf{3 0}$ and alkyne $\mathbf{2 3}$ was isolated, along with $425 \mathrm{mg}$ ( $82 \%$ yield) of alkynyl alcohol 39. A larger quantity of alkynyl alcohol $39(1.57 \mathrm{~g})$ was prepared in similar unoptimized protocol from 23 and $\mathbf{3 0}$ in $44 \%$ yield.

${ }^{1} \mathrm{H} \mathrm{NMR}\left(\mathrm{CDCl}_{3}, 600 \mathrm{MHz}\right): \delta 7.28(\mathrm{~m}, 4 \mathrm{H}), 6.87(\mathrm{~m}, 4 \mathrm{H}), 5.68(\mathrm{dd}, 1 \mathrm{H}, J=15.6,7.8 \mathrm{~Hz})$, $5.45(\mathrm{dd}, 1 \mathrm{H}, J=15.6,6.0 \mathrm{~Hz}), 4.74(\mathrm{~d}, 1 \mathrm{H}, J=11.4 \mathrm{~Hz}), 4.69(\mathrm{~m}, 1 \mathrm{H}), 4.48(\mathrm{~s}, 2 \mathrm{H}), 4.43$ (d, $1 \mathrm{H}, J=11.4 \mathrm{~Hz}), 4.36(\mathrm{~m}, 1 \mathrm{H}), 4.29(\mathrm{~m}, 1 \mathrm{H}), 4.14(\mathrm{~m}, 1 \mathrm{H}), 3.81(\mathrm{~s}, 3 \mathrm{H}), 3.80(\mathrm{~s}, 3 \mathrm{H})$, $2.96(\mathrm{t}, 1 \mathrm{H}, J=6.0 \mathrm{~Hz}), 2.54(\mathrm{q}, 1 \mathrm{H}), 2.42(\mathrm{~m}, 3 \mathrm{H}), 1.98(\mathrm{~m}, 1 \mathrm{H}), 1.92(\mathrm{~m}, 1 \mathrm{H}), 1.82(\mathrm{~m}$, $1 \mathrm{H}), 1.69(\mathrm{~m}, 1 \mathrm{H}), 1.48(\mathrm{~s}, 3 \mathrm{H}), 1.47(\mathrm{~s}, 3 \mathrm{H}), 1.07$ (d, 3H, J=6.6 Hz), 0.95 (d, 6H, J=6.6 $\mathrm{Hz}) \cdot{ }^{13} \mathrm{C} \mathrm{NMR}\left(\mathrm{CDCl}_{3}, 150 \mathrm{MHz}\right): \delta 159.6,159.2,136.7,131.3,129.9,129.5,129.2$, 114.0, 113.8, 103.8, 99.4, 91.9, 88.6, 81.2, 75.0, 70.6, 70.0, 67.2, 66.8, 60.5, 55.4, 46.4, 41.4, 39.8, 38.0, 31.1, 30.3, 20.5, 19.6, 17.8, 15.5. IR (neat, $\left.\mathrm{cm}^{-1}\right): 3450(\mathrm{~m}, \mathrm{br}), 2960$ (s), 2935 (s), 2870 (s), 2170 (w), 1615 (s), 1590 (m), 1515 (s), 1465 (m), 1380 (s), 1300 (m), 1250 (s), 1200 (s), 1170 (s), 1080 (s, br), 1035 (s), 970 (m), 950 (m), 845 (s), 760 (m). HRMS (FAB+): Calcd. for $\mathrm{C}_{41} \mathrm{H}_{58} \mathrm{O}_{7} \mathrm{SiLi}\left([\mathrm{M}+\mathrm{Li}]^{+}\right)$, 697.4112. Found, 697.4147. Anal. Calcd. for $\mathrm{C}_{41} \mathrm{H}_{58} \mathrm{O}_{7} \mathrm{Si}$ : C, 71.27; $\mathrm{H}, 8.46$. Found: $\mathrm{C}, 71.02 ; \mathrm{H}, 8.45$. [ $\left.\alpha\right]_{\mathrm{D}}-18$ (c $\left.0.480, \mathrm{CHCl}_{3}\right)$. 
Hydromethylsilylether 40. Alkynyl alcohol $39(1.49 \mathrm{~g}, 2.17 \mathrm{mmol})$ was dissolved in $\left(\mathrm{Me}_{2} \mathrm{SiH}\right) \mathrm{NH}(2.3 \mathrm{~mL}, 13.0 \mathrm{mmol})$ and heated at $100^{\circ} \mathrm{C}$ for $15 \mathrm{~h}$, then the reaction was connected to the vacuum for $4 \mathrm{~h}$, producing crude $\mathbf{4 0}$ which was taken to the next step without purification.

${ }^{1} \mathrm{H} \mathrm{NMR}\left(\mathrm{CDCl}_{3}, 600 \mathrm{MHz}\right): \delta 7.28(\mathrm{~m}, 4 \mathrm{H}), 6.88(\mathrm{~m}, 4 \mathrm{H}), 5.67(\mathrm{dd}, 1 \mathrm{H}, J=15.6,8.4 \mathrm{~Hz})$, $5.45(\mathrm{dd}, 1 \mathrm{H}, J=15.6,6.6 \mathrm{~Hz}), 4.73(\mathrm{~d}, 1 \mathrm{H}, J=10.8 \mathrm{~Hz}), 4.65(\mathrm{~m}, 2 \mathrm{H}), 4.48(\mathrm{~s}, 2 \mathrm{H}), 4.39$ $(\mathrm{d}, 1 \mathrm{H}, J=10.8 \mathrm{~Hz}), 4.26(\mathrm{~m}, 2 \mathrm{H}), 4.06(\mathrm{~m}, 1 \mathrm{H}), 3.81(\mathrm{~s}, 3 \mathrm{H}), 3.80(\mathrm{~s}, 3 \mathrm{H}), 2.96(\mathrm{t}, 1 \mathrm{H}$, $J=6.0 \mathrm{~Hz}), 2.42(\mathrm{~m}, 3 \mathrm{H}), 2.10(\mathrm{~m}, 1 \mathrm{H}), 1.83(\mathrm{~m}, 2 \mathrm{H}), 1.67(\mathrm{~m}, 2 \mathrm{H}), 1.48(\mathrm{~s}, 3 \mathrm{H}), 1.46(\mathrm{~s}$, $3 \mathrm{H}), 1.07(\mathrm{~d}, 3 \mathrm{H}, J=6.6 \mathrm{~Hz}$ ), $0.95(\mathrm{~d}, 6 \mathrm{H}, J=6.6 \mathrm{~Hz}), 0.20(\mathrm{~s}, 9 \mathrm{H}), 0.19(\mathrm{~d}, 3 \mathrm{H}, J=1.8$ $\mathrm{Hz}), 0.16(\mathrm{~d}, 3 \mathrm{H}, J=1.8 \mathrm{~Hz}) .{ }^{13} \mathrm{C}$ NMR $\left(\mathrm{CDCl}_{3}, 150 \mathrm{MHz}\right): \delta 159.4,159.2,136.7,131.4$, 130.2, 129.9, 129.5, 129.3, 113.9, 113.8, 105.0, 99.4, 90.7, 88.6, 82.0, 80.9, 75.1, 70.4, $68.7,65.6,60.6,55.5,43.4,39.8,37.9,31.2,30.4,28.1,20.6,19.6,17.8,15.6,0.1,-0.7$, -0.9. IR (neat, $\mathrm{cm}^{-1}$ ): 2960 (s, br), 2935 (s, br), $2870(\mathrm{~s}), 2170(\mathrm{~m}), 2115$ (m, br), 1610 (s), $1515(\mathrm{~s}), 1465(\mathrm{~m}), 1380(\mathrm{~m}), 1300(\mathrm{~m}), 1250$ (s), $1170(\mathrm{~s}), 1080$ (s, br), $1040(\mathrm{~m})$, 905 (s), 845 (s), 760 (m). HRMS (FAB+): Calcd. for $\mathrm{C}_{43} \mathrm{H}_{64} \mathrm{O}_{7} \mathrm{Si}_{2} \mathrm{Li}\left([\mathrm{M}+\mathrm{Li}]^{+}\right), 755.4351$. Found, 755.4382.

$\beta$-hydroxyketone 42 and $\mathbf{4 3}$. To a solution of crude hydrodimethylsilyl ether $\mathbf{4 0}$ (ca. $2.17 \mathrm{mmol})$ in THF (10 mL), a solution of platinum(0)-1, 3-divinyl-1, 1, 3, 3tetramethyldisiloxane complex in xylenes $(70 \mu \mathrm{L})$ was added. The reaction was stirred at ambient temperature for $21 \mathrm{~h}$, producing THF solution of cyclic siloxane 41 (an aliquot of this solution was concentrated and spectroscopic characteristics were recorded). A THF solution of the siloxane was diluted with methanol (15 $\mathrm{mL}) ; \mathrm{KHCO}_{3}$ (650 $\mathrm{mg}, 6.61 \mathrm{mmol})$ and $\mathrm{KF}(380 \mathrm{mg}, 6.51 \mathrm{mmol})$ were added followed by slow addition of $30 \%$ aqueous $\mathrm{H}_{2} \mathrm{O}_{2}(12.5 \mathrm{~mL}, 108 \mathrm{mmol})$. After stirring at room temperature for $23 \mathrm{~h}$, the reaction was poured into cooled saturated $\mathrm{Na}_{2} \mathrm{SO}_{3}(\mathrm{aq})$, vigorously stirred for 2- $3 \mathrm{~h}$ and extracted with ether. Organic fractions were dried with $\mathrm{Na}_{2} \mathrm{SO}_{4}$, filtered, concentrated and chromatographed with pentane - ether $(1: 1)$ producing TMS unprotected alkynyl $\beta$-hydroxyketone 43 (207 mg, 13\% yield) and $\beta$-hydroxyketone 42 (600 mg, 43\% yield).

Cyclic siloxane (41): ${ }^{1} \mathrm{H}$ NMR $\left(\mathrm{CDCl}_{3}, 600 \mathrm{MHz}\right): \delta 7.29$ (d, $\left.4 \mathrm{H}, J=7.8 \mathrm{~Hz}\right), 6.86(\mathrm{~d}, 4 \mathrm{H}$, $J=7.8 \mathrm{~Hz}), 5.76(\mathrm{~m}, 1 \mathrm{H}), 5.70(\mathrm{dd}, 1 \mathrm{H}, J=15.6,8.4 \mathrm{~Hz}), 5.48(\mathrm{dd}, 1 \mathrm{H}, J=15.6,6.6 \mathrm{~Hz})$, 
$4.73(\mathrm{~d}, 1 \mathrm{H}, J=10.8 \mathrm{~Hz}), 4.59(\mathrm{~m}, 1 \mathrm{H}), 4.49(\mathrm{~d}, 2 \mathrm{H}, J=4.8 \mathrm{~Hz}), 4.45(\mathrm{~d}, 1 \mathrm{H}, J=10.8 \mathrm{~Hz})$, $4.35(\mathrm{~m}, 2 \mathrm{H}), 4.24(\mathrm{~m}, 1 \mathrm{H}), 3.80(\mathrm{~s}, 6 \mathrm{H}), 2.98(\mathrm{t}, 1 \mathrm{H}, J=6.0 \mathrm{~Hz}), 2.63(\mathrm{~m}, 1 \mathrm{H}), 2.44(\mathrm{~m}$, 1H), $2.22(\mathrm{~m}, 1 \mathrm{H}), 1.96(\mathrm{~m}, 1 \mathrm{H}), 1.86(\mathrm{~m}, 2 \mathrm{H}), 1.52(\mathrm{~s}, 3 \mathrm{H}), 1.46(\mathrm{~s}, 3 \mathrm{H}), 1.08(\mathrm{~d}, 3 \mathrm{H}, J=$ $6.6 \mathrm{~Hz}), 0.94(\mathrm{~d}, 6 \mathrm{H}, J=7.2 \mathrm{~Hz}) .{ }^{13} \mathrm{C}$ NMR $(\mathrm{CDCl} 3,150 \mathrm{MHz}): \delta 159.3,159.2,141.5$, $137.7,136.2,131.4,130.4,129.8,129.7,129.4,113.8,105.3,98.9,90.3,88.6,75.0$, 73.0, 70.7, 69.9, 68.3, 66.0, 55.4, 44.8, 39.8, 37.6, 36.4, 31.1, 30.5, 20.5, 20.0, 17.8, 15.5, 1.0, 0.1, -0.2. IR (neat, $\mathrm{cm}^{-1}$ ): 2960 (s, br), 2870 (s, br), $2170(\mathrm{w}), 1615$ (m), 1515 (s), $1465(\mathrm{~m}), 1380(\mathrm{~m}), 1250$ (s), $1170(\mathrm{~m}), 1070$ (s, br), 1040 (s), 845 (s, br), $785(\mathrm{~m})$. HRMS (FAB+): Calcd. for $\mathrm{C}_{43} \mathrm{H}_{64} \mathrm{O}_{7} \mathrm{Si}_{2} \mathrm{Li}\left([\mathrm{M}+\mathrm{Li}]^{+}\right)$, 755.4351. Found, 755.4333.

42: ${ }^{1} \mathrm{H}$ NMR $\left(\mathrm{CDCl}_{3}, 600 \mathrm{MHz}\right): \delta 7.28(\mathrm{~d}, 4 \mathrm{H}), 6.88(\mathrm{~m}, 4 \mathrm{H}), 5.66(\mathrm{dd}, 1 \mathrm{H}, J=15.6,7.8$ $\mathrm{Hz}), 5.44(\mathrm{dd}, 1 \mathrm{H}, J=15.6,6.6 \mathrm{~Hz}), 4.73(\mathrm{~d}, 1 \mathrm{H}, J=10.8 \mathrm{~Hz}), 4.48(\mathrm{~s}, 2 \mathrm{H}), 4.43(\mathrm{~d}, 1 \mathrm{H}$, $J=10.8 \mathrm{~Hz}), 4.40-4.30(\mathrm{~m}, 4 \mathrm{H}), 3.80(\mathrm{~s}, \mathrm{br}, 6 \mathrm{H}), 3.20(\mathrm{~s}, \mathrm{br}, 1 \mathrm{H},-\mathrm{OH}), 2.96(\mathrm{t}, 1 \mathrm{H}, J=5.4$ $\mathrm{Hz}), 2.69(\mathrm{dd}, 1 \mathrm{H}, J=15.6,7.8 \mathrm{~Hz}), 2.58(\mathrm{~m}, 2 \mathrm{H}), 2.42(\mathrm{~m}, 3 \mathrm{H}), 1.85(\mathrm{~m}, 2 \mathrm{H}), 1.82(\mathrm{~m}$, $2 \mathrm{H}), 1.46(\mathrm{~s}, 3 \mathrm{H}), 1.38(\mathrm{~s}, 3 \mathrm{H}), 1.07(\mathrm{~d}, 3 \mathrm{H}, \mathrm{J}=7.2 \mathrm{~Hz}), 0.94(\mathrm{~m}, 6 \mathrm{H}) .{ }^{13} \mathrm{C} \mathrm{NMR}(\mathrm{CDCl} 3$, $150 \mathrm{MHz}): \delta 209.3,159.5,159.2,136.3,131.4,130.0,129.6,129.5,113.9,113.8$, $104.4,99.0,91.4,88.6,75.0,70.7,70.1,66.0,65.8,64.6,55.4,50.9,49.8,42.2,39.8$, 37.0, 31.1, 30.2, 20.6, 19.9, 17.8, 15.6, 0.1. IR (neat, $\mathrm{cm}^{-1}$ ): 3420 (s, br), 2960 (s), 2915 (s), 2875 (s), 2170 (w), $1710(\mathrm{~m}), 1610$ (s), 1515 (s), $1460(\mathrm{~m}), 1380$ (s), 1300 (m), 1250 (s), 1179 (s), 1090 (s), 8445 (m). HRMS (FAB+): Calcd. for $\mathrm{C}_{41} \mathrm{H}_{60} \mathrm{O}_{8} \mathrm{SiLi}\left([\mathrm{M}+\mathrm{Li}]^{+}\right)$, 715.4218. Found, 715. 4210. Anal. Calcd. for $\mathrm{C}_{41} \mathrm{H}_{60} \mathrm{O}_{8} \mathrm{Si}$ : C, 69.48; $\mathrm{H}, 8.53$. Found: $\mathrm{C}$, 68.99; $\mathrm{H}, 8.53$. $[\alpha]_{D}-28$ (c $\left.0.580, \mathrm{CHCl}_{3}\right)$

43: ${ }^{1} \mathrm{H}$ NMR ( $\left.\mathrm{CDCl}_{3}, 600 \mathrm{MHz}\right): \delta 7.28(\mathrm{~m}, 4 \mathrm{H}), 6.88(\mathrm{~m}, 4 \mathrm{H}), 5.66(\mathrm{dd}, 1 \mathrm{H}, J=15.6,8.4$ $\mathrm{Hz}), 5.44(\mathrm{dd}, 1 \mathrm{H}, J=15.6,6.6 \mathrm{~Hz}), 4.76(\mathrm{~d}, 1 \mathrm{H}, J=10.8 \mathrm{~Hz}), 4.48(\mathrm{~s}, 2 \mathrm{H}), 4.43(\mathrm{~d}, 1 \mathrm{H}$, $J=10.8 \mathrm{~Hz}), 4.37(\mathrm{~m}, 4 \mathrm{H}), 3.806(\mathrm{~s}, 3 \mathrm{H}), 3.802(\mathrm{~s}, 3 \mathrm{H}), 3.21(\mathrm{~s}, \mathrm{br}, 1 \mathrm{H},-\mathrm{OH}), 2.96(\mathrm{t}$, $1 \mathrm{H}, J=6.0 \mathrm{~Hz}), 2.70(\mathrm{dd}, 1 \mathrm{H}, J=15.6,7.2 \mathrm{~Hz}), 2.58(\mathrm{~m}, 2 \mathrm{H}), 2.51(\mathrm{~d}, 1 \mathrm{H}, J=2.4 \mathrm{~Hz})$, $2.42(\mathrm{~m}, 2 \mathrm{H}), 1.87(\mathrm{~m}, 3 \mathrm{H}), 1.83(\mathrm{~m}, 2 \mathrm{H}), 1.47(\mathrm{~s}, 3 \mathrm{H}), 1.38(\mathrm{~s}, 3 \mathrm{H}), 1.07(\mathrm{~d}, 3 \mathrm{H}, J=6.6$ $\mathrm{Hz}), 0.94(\mathrm{~m}, 6 \mathrm{H}) .{ }^{13} \mathrm{C} \mathrm{NMR}\left(\mathrm{CDCl}_{3}, 150 \mathrm{MHz}\right): \delta 209.3,159.6,159.2,136.3,131.4$, 130.0, 129.6, 129.5, 114.0, 113.8, 99.0, 88.6, 82.6, 75.0, 74.4, 70.8, 70.1, 65.8, 65.4, $64.4,55.4,50.8,49.7,42.1,39.7,37.0,31.1,30.2,20.5,19.9,17.8,15.5$. IR (neat, $\mathrm{cm}^{-}$ 1): 3450 (s, br), 3290 (s, br), 2960 (s, br), 2870 (s, br), 1710 (s), 1610 (s), 1515 (s), 1465 
(s), 1380 (s), 1300 (s), 1250 (s, br), 1175 (s), 1085 (s, br), 1040 (s, br), 975 (s), 825 (s). HRMS (FAB+): Calcd. for $\mathrm{C}_{38} \mathrm{H}_{52} \mathrm{O}_{8} \mathrm{Li}\left([\mathrm{M}+\mathrm{Li}]^{+}\right)$, 643.3822. Found, 643.3846. Anal. Calcd. for $\mathrm{C}_{38} \mathrm{H}_{52} \mathrm{O}_{8}: \mathrm{C}, 71.67 ; \mathrm{H}, 8.23$. Found: $\mathrm{C}, 70.87 ; \mathrm{H}, 8.32$. [ $\left.\alpha\right]_{D}-13$ (c 0.590 , $\left.\mathrm{CHCl}_{3}\right)$.

Diacetonide 44. To a solution of a mixture of $\beta$-hydroxyketones 42 (193 mg, 0.27 $\mathrm{mmol})$ and $43(588 \mathrm{mg}, 0.92 \mathrm{mmol})$ in THF $(15 \mathrm{~mL})$, methanol $(770 \mu \mathrm{L})$ was added at $-78^{\circ} \mathrm{C}$ followed by $1 \mathrm{M}$ solution of $\mathrm{Et}_{2} \mathrm{BOMe}$ in THF $(1.3 \mathrm{~mL}, 1.31 \mathrm{mmol})$. After $3.5 \mathrm{~h}$ of stirring, solid $\mathrm{NaBH}_{4}$ (90 mg, $2.38 \mathrm{mmol}$ ) was added. The reaction was stirred for $11.5 \mathrm{~h}$ at $-78^{\circ} \mathrm{C}$, and then $30 \%$ aqueous $\mathrm{H}_{2} \mathrm{O}_{2}(10 \mathrm{~mL})$ was added. The reaction was allowed to warm to room temperature, stirred for $3 \mathrm{~h}$ and quenched with saturated aqueous $\mathrm{Na}_{2} \mathrm{SO}_{3}$ at $0^{\circ} \mathrm{C}$. The reaction was stirred for $1 \mathrm{~h}$ at room temperatures and extracted with ether. Organic fractions were dried with $\mathrm{Na}_{2} \mathrm{SO}_{4}$, filtered, and concentrated to give the crude diol. For removal of the alkynylsilyl group, the residue was dissolved in methanol $(5 \mathrm{~mL})$ and treated with $\mathrm{K}_{2} \mathrm{CO}_{3}(90 \mathrm{mg}, 0.65 \mathrm{mmol})$. In $2 \mathrm{~h}$, additional $\mathrm{K}_{2} \mathrm{CO}_{3}$ (90 $\mathrm{mg}, 0.65 \mathrm{mmol}$ ) was added. The reaction was vigorously stirred for $20 \mathrm{~h}$ at room temperature, then diluted with water and extracted with ether. Organic fractions were dried with $\mathrm{Na}_{2} \mathrm{SO}_{4}$, filtered and concentrated yielding the crude terminal alkynyl diol. To a solution of this intermediate (ca. $1.19 \mathrm{mmol}$ ) in 2, 2-dimethoxypropane $(5 \mathrm{~mL}$ ), $\mathrm{TsOH} \cdot \mathrm{H}_{2} \mathrm{O}(30 \mathrm{mg}, 0.16 \mathrm{mmol})$ and $3 \AA$ molecular sieves $(300 \mathrm{mg})$ were added. The reaction was stirred at room temperature for $21 \mathrm{~h}$, diluted with $E t_{3} \mathrm{~N}$, filtered and concentrated. The residue was chromatographed on silica gel pretreated with $2.5 \mathrm{vol} \%$ of $\mathrm{Et}_{3} \mathrm{~N}$ with pentane - ether $(75: 25)$. Diacetonide 44 was obtained in $67 \%$ yield (545 $\mathrm{mg}$, three steps starting from the mixture of $\mathbf{4 2}$ and $\mathbf{4 3}$ ).

${ }^{1} \mathrm{H}$ NMR $\left(\mathrm{CDCl}_{3}, 600 \mathrm{MHz}\right): \delta 7.28(\mathrm{~d}, \mathrm{br}, 4 \mathrm{H}), 6.88(\mathrm{~m}, 4 \mathrm{H}), 5.65(\mathrm{dd}, 1 \mathrm{H}, J=15.6,8.4$ $\mathrm{Hz}), 5.46(\mathrm{dd}, 1 \mathrm{H}, J=15.6,6.0 \mathrm{~Hz}), 4.72(\mathrm{~d}, 1 \mathrm{H}, J=11.4 \mathrm{~Hz}), 4.48(\mathrm{~s}, 2 \mathrm{H}), 4.43(\mathrm{~d}, 1 \mathrm{H}$, $J=11.4 \mathrm{~Hz}), 4.30(\mathrm{~m}, 2 \mathrm{H}), 4.08(\mathrm{~m}, 3 \mathrm{H}), 3.81(\mathrm{~s}, 3 \mathrm{H}), 3.80(\mathrm{~s}, 3 \mathrm{H}), 2.96(\mathrm{t}, 1 \mathrm{H}, J=5.4$ $\mathrm{Hz}), 2.44(\mathrm{~s}, 1 \mathrm{H}), 2.43(\mathrm{~m}, 1 \mathrm{H}), 1.88(\mathrm{~m}, 1 \mathrm{H}), 1.82(\mathrm{~m}, 2 \mathrm{H}), 1.48(\mathrm{~m}, 3 \mathrm{H}), 1.45(\mathrm{~s}, 3 \mathrm{H})$, $1.43(\mathrm{~m}, 3 \mathrm{H}), 1.41(\mathrm{~s}, 3 \mathrm{H}), 1.36(\mathrm{~s}, 3 \mathrm{H}), 1.32(\mathrm{~s}, 3 \mathrm{H}), 1.07$ (d, 3H, J=6.6 Hz), 0.94 (d, $6 \mathrm{H}, J=7.2 \mathrm{~Hz}) .{ }^{13} \mathrm{C} \mathrm{NMR}\left(\mathrm{CDCl}_{3}, 150 \mathrm{MHz}\right): \delta 159.5,159.2,136.0,131.4,130.1,129.9$, $129.5,113.9,113.8,98.74,98.74,98.70,88.7,83.3,75.0,73.5,70.6,65.0,64.9,64.8$, 
63.9, 55.5, 43.1, 42.9, 39.7, 37.8, 37.6, 31.1, 30.5, 30.4, 20.5, 20.1, 17.9, 15.5. IR (neat, $\mathrm{cm}^{-1}$ ): 3290 (m, br), 2955 (s, br), 2870 (s), 1610 (s), 1585 (m), 1515 (s), 1465 (s), 1380 (s), 1300 (m), 1250 (s), 1200 (s), 1170 (s), 1105 (s), 1075 (s), 1035 (s), 975 (m), 938 (s), $820(\mathrm{~s})$.

Alkynyl alcohol 45. To a solution of alkyne 44 (175 mg, $0.176 \mathrm{mmol})$ in THF $(4 \mathrm{~mL})$ a solution of $n$-BuLi $(330 \mu \mathrm{L}, 0.85 \mathrm{mmol})$ in hexane was added at $-78^{\circ} \mathrm{C}$. The reaction was stirred $2.5 \mathrm{~h}$ at $-78 \rightarrow-40^{\circ} \mathrm{C}$, then $\mathrm{BF}_{3} \cdot \mathrm{OEt}_{2}(40 \mu \mathrm{L}, 0.86 \mathrm{mmol})$ was added at $-78^{\circ} \mathrm{C}$. The reaction was stirred $10 \mathrm{~min}$ at $-78^{\circ} \mathrm{C}$ and a solution of epoxide $36(126 \mathrm{mg}$, $0.39 \mathrm{mmol})$ in THF $(2 \mathrm{~mL})$ was added via cannula. The mixture was stirred at $-78^{\circ} \mathrm{C}$ for $3 \mathrm{~h}$, then diluted with saturated aqueous $\mathrm{NaCl}$ and extracted with ether. Organic fractions were dried with $\mathrm{MgSO}_{4}$, filtered and concentrated. After chromatography of the residue with pentane - ether (1:1) $143 \mathrm{mg}$ of a mixture of starting epoxide 36 and alkyne $\mathbf{4 4}$ was isolated along with $178 \mathrm{mg}$ (59\% yield) of alkynyl alcohol 45 .

${ }^{1} \mathrm{H}$ NMR $\left(\mathrm{CDCl}_{3}, 600 \mathrm{MHz}\right): \delta 7.27(\mathrm{~m}, 6 \mathrm{H}), 6.88(\mathrm{~m}, 6 \mathrm{H}), 5.65(\mathrm{dd}, 1 \mathrm{H}, J=15.6,7.8 \mathrm{~Hz})$, $5.46(\mathrm{dd}, 1 \mathrm{H}, J=15.6,6.0 \mathrm{~Hz}), 4.71(\mathrm{~d}, 1 \mathrm{H}, J=10.8 \mathrm{~Hz}), 4.68(\mathrm{~d}, 1 \mathrm{H}, J=10.8 \mathrm{~Hz}), 4.48$ $(\mathrm{s}, 2 \mathrm{H}), 4.46(\mathrm{~d}, 1 \mathrm{H}, J=10.8 \mathrm{~Hz}), 4.39(\mathrm{~d}, 1 \mathrm{H}, J=10.8 \mathrm{~Hz}), 4.30(\mathrm{~m}, 2 \mathrm{H}), 4.07(\mathrm{~m}, 3 \mathrm{H})$, $3.93(\mathrm{~m}, 1 \mathrm{H}), 3.809(\mathrm{~s}, 3 \mathrm{H}), 3.805(\mathrm{~s}, 6 \mathrm{H}), 3.73(\mathrm{~m}, 1 \mathrm{H}), 2.96(\mathrm{t}, 1 \mathrm{H}, J=5.4 \mathrm{~Hz}), 2.61(\mathrm{dd}$, $1 \mathrm{H}, J=16.8,4.8 \mathrm{~Hz}), 2.49(\mathrm{dd}, 1 \mathrm{H}, J=16.8,6.6 \mathrm{~Hz}), 2.43(\mathrm{~m}, 3 \mathrm{H}), 1.99(\mathrm{~m}, 1 \mathrm{H}), 1.86-$ $1.75(\mathrm{~m}, 4 \mathrm{H}), 1.48(\mathrm{~m}, 3 \mathrm{H}), 1.44(\mathrm{~s}, 3 \mathrm{H}), 1.42(\mathrm{~m}, 3 \mathrm{H}), 1.41(\mathrm{~s}, 3 \mathrm{H}), 1.35(\mathrm{~s}, 3 \mathrm{H}), 1.31$ $(\mathrm{s}, 3 \mathrm{H}), 1.07(\mathrm{~d}, 3 \mathrm{H}, \mathrm{J}=7.2 \mathrm{~Hz}), 0.94(\mathrm{~d}, 6 \mathrm{H}, \mathrm{J}=6.6 \mathrm{~Hz}), 0.16(\mathrm{~s}, 9 \mathrm{H}) \cdot{ }^{13} \mathrm{C} \mathrm{NMR}\left(\mathrm{CDCl}_{3}\right.$, $150 \mathrm{MHz}): \delta 159.6,159.4,159.2,136.0,131.4,130.3,130.1,129.9,129.8,129.5$, $114.1,113.9,113.8,103.2,98.8,98.7,88.7,87.5,82.2,81.7,75.0,71.2,70.6,70.5$, 69.8, 65.14, 65.07, 64.8, 64.4, 58.6, 55.5, 43.4, 43.2, 40.4, 39.7, 37.8, 37.7, 31.2, 30.5, 30.4, 27.7, 25.7, 20.5, 20.1, 18.6, 17.9, 15.5, 0.3. IR (neat, $\left.\mathrm{cm}^{-1}\right): 3475(\mathrm{~m}, \mathrm{br}), 2955$ (s, br), $2870(\mathrm{~s}), 2175(\mathrm{~m}), 1610(\mathrm{~s}), 1580(\mathrm{~m}), 1515(\mathrm{~s}), 1465(\mathrm{~m}), 1380(\mathrm{~s}), 1300(\mathrm{~m})$, 1250 (s), 1170 (s), 1085 (s, br), 1035 (s), 975 (m), 940 (m), 845 (s). HRMS (FAB+): Calcd. for $\mathrm{C}_{59} \mathrm{H}_{84} \mathrm{O}_{11} \mathrm{SiLi}\left([\mathrm{M}+\mathrm{Li}]^{+}\right)$, 1003.5943. Found, 1003.5900. Anal. Calcd. for $\mathrm{C}_{59} \mathrm{H}_{84} \mathrm{O}_{11} \mathrm{Si}: \mathrm{C}, 71.05 ; \mathrm{H}, 8.49$. Found: $\mathrm{C}, 70.83 ; \mathrm{H}, 8.76$. $[\alpha]_{D}-5$ (c $0.815, \mathrm{CHCl}_{3}$ ). 
Hydrodimethylsilylether of 45 . The above hydrodimethylsilyl ether was prepared from alkynyl alcohol $\mathbf{4 5}$ in analogy with the preparation of $\mathbf{4 0}$ from 39.

${ }^{1} \mathrm{H} \mathrm{NMR}\left(\mathrm{CDCl}_{3}, 600 \mathrm{MHz}\right): \delta 7.24(\mathrm{~m}, 6 \mathrm{H}), 6.83(\mathrm{~m}, 6 \mathrm{H}), 5.62(\mathrm{dd}, 1 \mathrm{H}, J=15.6,7.8 \mathrm{~Hz})$, $5.43(\mathrm{dd}, J=15.6,6.6 \mathrm{~Hz}), 4.66(\mathrm{~d}, 1 \mathrm{H}, J=10.8 \mathrm{~Hz}), 4.55$ (d, $1 \mathrm{H}, J=10.8 \mathrm{~Hz}), 4.45$ (s, 2H), $4.4(\mathrm{~d}, 1 \mathrm{H}, J=10.8 \mathrm{~Hz}), 4.37(\mathrm{~d}, 1 \mathrm{H}, J=11.4 \mathrm{~Hz}), 4.26(\mathrm{~m}, 2 \mathrm{H}), 4.04(\mathrm{~m}, 4 \mathrm{H}), 3.96$ (m, 1H), $3.77(\mathrm{~s}, \mathrm{br}, 6 \mathrm{H}), 3.76(\mathrm{~s}, 3 \mathrm{H}), 3.64(\mathrm{~m}, 1 \mathrm{H}), 2.93(\mathrm{t}, 1 \mathrm{H}, J=4.8 \mathrm{~Hz}), 2.53-2.32$ $(\mathrm{m}, 6 \mathrm{H}), 1.88(\mathrm{~m}, 2 \mathrm{H}), 1.78(\mathrm{~m}, 2 \mathrm{H}), 1.44(\mathrm{~m}, 3 \mathrm{H}), 1.41(\mathrm{~s}, 3 \mathrm{H}), 1.39(\mathrm{~m}, 3 \mathrm{H}), 1.37(\mathrm{~s}$, $3 \mathrm{H}), 1.31(\mathrm{~s}, 3 \mathrm{H}), 1.28(\mathrm{~s}, 3 \mathrm{H}), 1.03(\mathrm{~d}, 3 \mathrm{H}, J=6.6 \mathrm{~Hz}), 0.90(\mathrm{~d}, 6 \mathrm{H}, J=6.6 \mathrm{~Hz}) \cdot{ }^{13} \mathrm{C}$ NMR $\left(\mathrm{CDCl}_{3}, 150 \mathrm{MHz}\right): \delta 159.4,159.3,159.2,136.0,131.4,130.7,130.4,130.1$, $129.9,129.52,129.48,113.94,113.89,113.86,104.0,98.8,98.7,88.7,88.0,86.7,82.8$, $75.0,74.1,70.9,70.6,70.4,70.1,65.1,64.7,64.3,55.5,43.4,43.2,41.2,39.7,37.8$, $37.7,31.1,30.5,30.4,27.5,25.8,20.5,20.1,17.9,15.5,0.3,-0.6,-0.7$.

$\beta$-hydroxyketone 46. The $\beta$-hydroxyketone was prepared from the above hydrodimethylsilyl ether in 65\% yield (two steps), in analogy with $\mathbf{4 2 .}$

${ }^{1} \mathrm{H}$ NMR $\left(\mathrm{CDCl}_{3}, 600 \mathrm{MHz}\right): \delta 7.27(\mathrm{~m}, 4 \mathrm{H}), 7.21(\mathrm{~d}, 2 \mathrm{H}, J=7.2 \mathrm{~Hz}), 6.86(\mathrm{~m}, 6 \mathrm{H}), 5.65$ (dd, $1 \mathrm{H}, J=15.6,8.4 \mathrm{~Hz}$ ), 5.46 (dd, $1 \mathrm{H}, J=15.6,6.0 \mathrm{~Hz}$ ), 4.65 (d, 1H, J=10.8 Hz), 4.48 $(\mathrm{m}, 3 \mathrm{H}), 4.43(\mathrm{~d}, 2 \mathrm{H}, J=10.8 \mathrm{~Hz}), 4.31(\mathrm{~d}, 2 \mathrm{H}, J=10.8 \mathrm{~Hz}), 4.23(\mathrm{~m}, 1 \mathrm{H}), 4.15(\mathrm{~m}, 1 \mathrm{H})$, 4.12- $4.03(\mathrm{~m}, 3 \mathrm{H}), 3.80(\mathrm{~s}, 3 \mathrm{H}), 3.79(\mathrm{~s}, 6 \mathrm{H}), 3.72(\mathrm{~m}, 1 \mathrm{H}, 3.56(\mathrm{~s}, \mathrm{br}, 1 \mathrm{H},-\mathrm{OH}), 2.96(\mathrm{t}$, $1 \mathrm{H}, J=4.8 \mathrm{~Hz}), 2.71(\mathrm{dd}, 1 \mathrm{H}, J=15.6,7.2 \mathrm{~Hz}), 2.61-2.53(\mathrm{~m}, 3 \mathrm{H}), 2.47-2.40(\mathrm{~m}, 3 \mathrm{H})$, $1.81(\mathrm{~m}, 1 \mathrm{H}), 1.76(\mathrm{~m}, 2 \mathrm{H}), 1.63(\mathrm{~m}, 2 \mathrm{H}), 1.53(\mathrm{~m}, 2 \mathrm{H}), 1.48(\mathrm{~m}, 2 \mathrm{H}), 1.45(\mathrm{~s}, 3 \mathrm{H}), 1.43$ (m, 2H), 1.41 (s, 3H), $1.39(\mathrm{~s}, 3 \mathrm{H}), 1.36(\mathrm{~s}, 3 \mathrm{H}), 1.06(\mathrm{~d}, 3 \mathrm{H}, J=6.6 \mathrm{~Hz}), 0.93(\mathrm{~d}, 6 \mathrm{H}, J=$ $6.6 \mathrm{~Hz}), 0.16(\mathrm{~s}, 9 \mathrm{H}) .{ }^{13} \mathrm{C} \mathrm{NMR}\left(\mathrm{CDCl}_{3}, 150 \mathrm{MHz}\right): \delta 209.4,159.5,159.4,159.2,136.0$, 131.4, 130.7, 130.1, 130.0, 129.7, 129.6, 129.5, 114.1, 114.0, 113.8, 103.4, 98.8, 98.7, 88.7, 87.3, 76.5, 75.0, 72.3, 71.8, 71.1, 70.6, 66.8, 65.8, 64.7, 55.4, 50.8, 49.7, 43.2, $42.7,40.9,39.7,38.0,37.8,31.1,30.5,25.5,20.5,20.15,20.09,17.9,15.5,0.21$. IR (neat, $\left.\mathrm{cm}^{-1}\right): 3490(\mathrm{~m}, \mathrm{br}), 2960$ (s), $2915(\mathrm{~s}), 2850(\mathrm{~s}), 2175(\mathrm{~m}), 1710(\mathrm{~m}), 1610(\mathrm{~m})$, $1580(\mathrm{~m}), 1515(\mathrm{~m}), 1460(\mathrm{~m}), 1380(\mathrm{~m}), 1260$ (m), 1100 (s, br). HRMS (FAB+): Calcd. for $\mathrm{C}_{59} \mathrm{H}_{86} \mathrm{O}_{12} \mathrm{SiLi}\left([\mathrm{M}+\mathrm{Li}]^{+}\right), 1021.6049$. Found, 1021.6061. Anal. Calcd. for $\mathrm{C}_{59} \mathrm{H}_{86} \mathrm{O}_{12} \mathrm{Si}$ : C, 69.79; $\mathrm{H}, 8.54$. Found: $\mathrm{C}, 70.13 ; \mathrm{H}, 8.73$. $[\alpha]_{\mathrm{D}}+28$ (c $\left.0.305, \mathrm{CHCl}_{3}\right)$. 
Triacetonide $\mathbf{4 7}$ was prepared in $80 \%$ yield (three steps) from $\mathbf{4 6}$, by a reaction sequence similar to that reported for the conversion of $\mathbf{4 2}$ to $\mathbf{4 4}$.

${ }^{1} \mathrm{H}$ NMR $\left(\mathrm{CDCl}_{3}, 600 \mathrm{MHz}\right): \delta 7.29-7.26(\mathrm{~m}, 6 \mathrm{H}), 6.90-6.86(\mathrm{~m}, 6 \mathrm{H}), 5.66(\mathrm{dd}, 1 \mathrm{H}, J=$ 15.6, 7.8 Hz), 5.47 (dd, $1 \mathrm{H}, J=15.6,6.6 \mathrm{~Hz}), 4.57(\mathrm{~d}, 1 \mathrm{H}, J=11.4 \mathrm{~Hz}), 4.48(\mathrm{~m}, 4 \mathrm{H})$, $4.42(\mathrm{~d}, 1 \mathrm{H}, J=11.4 \mathrm{~Hz}), 4.32(\mathrm{~m}, 1 \mathrm{H}), 4.13-4.08(\mathrm{~m}, 3 \mathrm{H}), 4.01(\mathrm{~m}, 2 \mathrm{H}), 3.84(\mathrm{~m}, 1 \mathrm{H})$, $3.812(\mathrm{~s}, 3 \mathrm{H}), 3.808(\mathrm{~s}, 3 \mathrm{H}), 3.78(\mathrm{~s}, 3 \mathrm{H}), 3.67(\mathrm{~m}, 1 \mathrm{H}), 2.97(\mathrm{t}, 1 \mathrm{H}, J=5.4 \mathrm{~Hz}), 2.48-$ $2.43(\mathrm{~m}, 3 \mathrm{H}), 2.02(\mathrm{~m}, 1 \mathrm{H}), 1.91(\mathrm{~m}, 1 \mathrm{H}), 1.82(\mathrm{~m}, 1 \mathrm{H}), 1.71(\mathrm{~m}, 1 \mathrm{H}), 1.59(\mathrm{~m}, 2 \mathrm{H}), 1.56-$ $1.49(\mathrm{~m}, 4 \mathrm{H}), 1.46(\mathrm{~s}, 3 \mathrm{H}), 1.42(\mathrm{~s}, \mathrm{br}, 9 \mathrm{H}), 1.39(\mathrm{~s}, 3 \mathrm{H}), 1.38(\mathrm{~s}, 3 \mathrm{H}), 1.30-1.26(\mathrm{~m}$, $4 \mathrm{H}), 1.13(\mathrm{~m}, 2 \mathrm{H}), 1.07(\mathrm{~d}, 3 \mathrm{H}, J=6.6 \mathrm{~Hz}), 0.95(\mathrm{~d}, 6 \mathrm{H}, J=6.6 \mathrm{~Hz}) \cdot{ }^{13} \mathrm{C}$ NMR $\left(\mathrm{CDCl}_{3}\right.$, $150 \mathrm{MHz}): \delta 159.4,159.3,159.2,136.0,131.4,131.2,130.5,130.1,129.7,129.48$, $129.43,114.0,113.9,113.8,98.74,98.66,98.59,88.7,81.2,75.0,72.9,72.3,70.8$, 70.6, 70.4, 66.2, 65.9, 65.6, 65.0, 64.8, 55.4, 43.2, 40.6, 39.7, 38.1, 37.8, 37.4, 31.1, 30.5, 23.9, 20.5, 20.21, 20.16, 20.09, 17.9, 15.5. IR (neat, $\mathrm{cm}^{-1}$ ): $3290(\mathrm{w}), 2990(\mathrm{~s})$, 2940 (s), 2915 (s), 2870 (s), $1610(\mathrm{~m}), 1510(\mathrm{~s}), 1465(\mathrm{~m}), 1380$ (s), $1300(\mathrm{~m}), 1250$ (s), 1200 (s), 1170 (s), 1105 (s), 1036 (s), 940 (m), 820 (m). HRMS (MALDI- DHB): m/z $1007.8\left([\mathrm{M}+\mathrm{Na}]^{+}\right), 967.8\left(\left[\mathrm{M}+\mathrm{Na}-\mathrm{C}_{3} \mathrm{H}_{4}\right]^{+}\right), 927.8\left(\left[\mathrm{M}+\mathrm{Na}-2\left(\mathrm{C}_{3} \mathrm{H}_{4}\right)\right]^{+}\right), 887.8([\mathrm{M}+\mathrm{Na}-$ $\left.\left.3\left(\mathrm{C}_{3} \mathrm{H}_{4}\right)\right]^{+}\right) \cdot[\alpha]_{\mathrm{D}}+28\left(\mathrm{c} 0.320, \mathrm{CHCl}_{3}\right)$.

Vinyl iodide 48. Attention! Preparation of $\mathrm{Cp}_{2} \mathrm{Zr}(\mathrm{H}) \mathrm{Cl}$ from $\mathrm{Cp}_{2} \mathrm{ZrCl}_{2}$ and $\mathrm{LiHBEt}_{3}$ yields unreproducible results on a small scale (starting from less then $60 \mathrm{mg}$ of $\mathrm{Cp}_{2} \mathrm{ZrCl}_{2}$ ). Additionally, this reduction works well only with a new bottle of Super-Hydride®. To a solution of $\mathrm{Cp}_{2} \mathrm{ZrCl}_{2}(100 \mathrm{mg}, 0.34 \mathrm{mmol})$ in THF $(1.0 \mathrm{~mL}), 1 \mathrm{M}$ THF solution of SuperHydride ${ }^{\circledR}(340 \mu \mathrm{L}, 0.34 \mathrm{mmol}$, freshly opened bottle) was added. The reaction was wrapped in aluminum foil and stirred at ambient temperature for $10 \mathrm{~min}$. After that, vigorous stirring was continued and an aliquot $(800 \mu \mathrm{L})$ of white suspension of $\mathrm{Cp}_{2} \mathrm{Zr}(\mathrm{H}) \mathrm{Cl}$ in THF was removed via syringe and disregarded. To the residual $\mathrm{Cp}_{2} \mathrm{Zr}(\mathrm{H}) \mathrm{Cl}$ (ca. $140.0 \mu \mathrm{mol}$ ) in THF (ca. $540 \mu \mathrm{L}$ ), a solution of alkyne 47 (40 mg, 40.0 $\mu \mathrm{mol}$ ) was injected. After $1.5 \mathrm{~h}$ of stirring at ambient temperature, 1.5 M THF solution of $\mathrm{I}_{2}$ was added dropwise until brown- red color persisted. The reaction was stirred additional 15 min, quenched with saturated aqueous $\mathrm{Na}_{2} \mathrm{SO}_{3}$ (color disappear) and extracted with ether. Combined organic fractions were dried with $\mathrm{Na}_{2} \mathrm{SO}_{4}$, filtered and 
concentrated. Vinyl iodide 48 was isolated in $90 \%$ yield $(40 \mathrm{mg})$ after flash chromatography on silica gel pretreated with 2.5 volume $\%$ of $\mathrm{Et}_{3} \mathrm{~N}$ with pentane - ether $(6: 4)$.

${ }^{1} \mathrm{H}$ NMR $\left(\mathrm{CDCl}_{3}, 600 \mathrm{MHz}\right): \delta$ 7.29- $7.24(\mathrm{~m}, 6 \mathrm{H}), 6.90-6.87(\mathrm{~m}, 6 \mathrm{H}), 6.54(\mathrm{~m}, 1 \mathrm{H}), 6.07$ (d, $1 \mathrm{H}, J=14.4 \mathrm{~Hz}$ ), $5.66(\mathrm{dd}, 1 \mathrm{H}, J=15.6,7.8 \mathrm{~Hz}), 5.47$ (dd, $1 \mathrm{H}, J=15.6,6.6 \mathrm{~Hz}), 4.51$ $4.45(\mathrm{~m}, 5 \mathrm{H}), 4.39(\mathrm{~d}, 1 \mathrm{H}, J=11.4 \mathrm{~Hz}), 4.32(\mathrm{~m}, 1 \mathrm{H}), 4.08(\mathrm{~m}, 4 \mathrm{H}), 4.00(\mathrm{~m}, 1 \mathrm{H}), 3.96$ $(\mathrm{m}, 1 \mathrm{H}), 3.811(\mathrm{~s}, 3 \mathrm{H}), 3.809(\mathrm{~s}, 3 \mathrm{H}), 3.79(\mathrm{~s}, 3 \mathrm{H}), 3.56(\mathrm{~m}, 1 \mathrm{H}), 2.97(\mathrm{t}, 1 \mathrm{H}, J=6.0 \mathrm{~Hz})$, $2.42(\mathrm{~m}, 1 \mathrm{H}), 2.35(\mathrm{~m}, 1 \mathrm{H}), 2.27(\mathrm{~m}, 1 \mathrm{H}), 1.83(\mathrm{~m}, 3 \mathrm{H}), 1.58(\mathrm{~m}, 4 \mathrm{H}), 1.56-1.48(\mathrm{~m}, 7 \mathrm{H})$, $1.46(\mathrm{~s}, 6 \mathrm{H}), 1.42(\mathrm{~s}, 9 \mathrm{H}), 1.393(\mathrm{~s}, 3 \mathrm{H}), 1.388(\mathrm{~s}, 3 \mathrm{H}), 1.07$ (d, 3H, J=6.6 Hz), 0.94 (d, $6 \mathrm{H}, J=6.6 \mathrm{~Hz}) .{ }^{13} \mathrm{C} \mathrm{NMR}\left(\mathrm{CDCl}_{3}, 150 \mathrm{MHz}\right): \delta 159.4,159.3,159.2,142.7,136.0,131.4$, 131.2, 130.6, 130.1, 129.7, 129.5, 129.4, 114.04, 113.99, 113.8, 98.76, 98.66, 98.60, $88.7,75.0,73.5,72.4,71.3,70.6,66.1,65.8,65.6,64.9,64.7,55.4,43.2,40.5,40.4$, $39.7,38.1,37.8,37.5,31.1,30.5$ (br), 20.5, 20.2, 20.1, 17.9, 15.5. IR (neat, $\mathrm{cm}^{-1}$ ): 2990 (s), 2940 (s), 2915 (s), 2870 (s), 1610 (s), 1510 (s), 1465 (s), 1380 (s), 1300 (s), 1250 (s), 1200 (s), 1170 (s), 1110 (s), 1040 (s), 940 (s), 820 (s). HRMS (FAB+): Calcd. for $\mathrm{C}_{59} \mathrm{H}_{85} \mathrm{IO}_{12} \mathrm{Na}\left([\mathrm{M}+\mathrm{Na}]^{+}\right), 1135.4983$. Found, 1135.4999. [ $\left.\alpha\right]_{D}+23$ (c 0.165, $\left.\mathrm{CHCl}_{3}\right)$.

Triol 49. The PMBn triether $48(24 \mathrm{mg}, 21.8 \mu \mathrm{mol})$ was dissolved in $\mathrm{CH}_{2} \mathrm{Cl}_{2}(6 \mathrm{~mL})$, and DDQ $(25 \mathrm{mg}, 109.0 \mu \mathrm{mol})$ was added in one portion. The reaction was shielded from light and stirred at room temperature for $45 \mathrm{~min}$, and then more DDQ (10 mg, 44.0 $\mu \mathrm{mol})$ was added. After $3 \mathrm{~h}$, the reaction was concentrated and chromatographed on silica gel pretreated with 2.5 volume\% of $\mathrm{Et}_{3} \mathrm{~N}$ with pentane - ether $(25: 75)$ producing triol 49 (10 $\mathrm{mg}, 61 \%$ yield).

${ }^{1} \mathrm{H}$ NMR $\left(\mathrm{CDCl}_{3}, 600 \mathrm{MHz}\right): \delta 6.57(\mathrm{~m}, 1 \mathrm{H}), 6.11(\mathrm{~d}, 1 \mathrm{H}, J=14.4 \mathrm{~Hz}), 5.63(\mathrm{dd}, 1 \mathrm{H}, J=$ 15.6, 7.2 Hz), 5.50 (dd, 1H, J= 15.6, 7.2 Hz), $4.34(\mathrm{~m}, 1 \mathrm{H}), 4.20-4.09(\mathrm{~m}, 6 \mathrm{H}), 3.89(\mathrm{~m}$, $1 \mathrm{H}), 3.56(\mathrm{~s}, 1 \mathrm{H},-\mathrm{OH}), 3.29(\mathrm{t}, \mathrm{br}, 1 \mathrm{H},-\mathrm{OH}), 3.16(\mathrm{t}, \mathrm{br}, 1 \mathrm{H},-\mathrm{OH}), 2.35(\mathrm{~m}, 1 \mathrm{H}), 2.26-$ $2.17(\mathrm{~m}, 2 \mathrm{H}), 1.72(\mathrm{~m}, 1 \mathrm{H}), 1.62(\mathrm{~m}, 4 \mathrm{H}), 1.57(\mathrm{~m}, 4 \mathrm{H}), 1.56-1.48(\mathrm{~m}, 6 \mathrm{H}), 1.450(\mathrm{~s}$, $3 \mathrm{H}), 1.444(\mathrm{~s}, 3 \mathrm{H}), 1.439(\mathrm{~s}, 3 \mathrm{H}), 1.40(\mathrm{~s}, 3 \mathrm{H}), 1.389(\mathrm{~s}, 3 \mathrm{H}), 1.385(\mathrm{~s}, 3 \mathrm{H}), 1.03(\mathrm{~d}, 3 \mathrm{H}$, $J=6.6 \mathrm{~Hz}), 0.92(\mathrm{dd}, 6 \mathrm{H}, J=12.6,6.6 \mathrm{~Hz}) .{ }^{13} \mathrm{C} \mathrm{NMR}\left(\mathrm{CDCl}_{3}, 150 \mathrm{MHz}\right): \delta 142.8,135.0$, 131.2, 99.1, 98.9, 98.81, 98.77, 98.74, 79.9, 70.6 (2C), 70.5, 67.5, 66.7, 65.3, 65.0, $64.7,43.9,43.3,43.1,42.7,42.2,39.4,37.9,37.4,37.1,30.7,30.5$ (2C), 30.3, 20.1, $20.0,19.9,19.8,17.3,13.9 .[\alpha]_{\mathrm{D}}+6\left(\mathrm{c} 0.501, \mathrm{CDCl}_{3}\right)$. 
Tetracetonide 50. To a solution of triol $49(10 \mathrm{mg}, 13.3 \mu \mathrm{mol})$ in 2, 2dimethoxypropane $(2 \mathrm{~mL})$, a solution of $\mathrm{TsOH}-\mathrm{H}_{2} \mathrm{O}(2 \mathrm{mg}, 11.0 \mu \mathrm{mol})$ in water $(20 \mu \mathrm{L})$ was added. The reaction was shielded from light and stirred at room temperature for 3 days, then diluted with $\mathrm{Et}_{3} \mathrm{~N}(0.1 \mathrm{~mL})$, concentrated and chromatographed on silica gel pretreated with 2.5 volume $\%$ of $\mathrm{Et}_{3} \mathrm{~N}$ with pentane - ether (1:1) yielding tetracetonide 50 (9.3 mg, 86\% yield).

${ }^{1} \mathrm{H}$ NMR $\left(\mathrm{CDCl}_{3}, 600 \mathrm{MHz}\right): \delta 6.53(\mathrm{~m}, 1 \mathrm{H}), 6.09(\mathrm{~d}, 1 \mathrm{H}, J=14.4 \mathrm{~Hz}), 5.63(\mathrm{dd}, 1 \mathrm{H}, J=$ 15.6, $6.6 \mathrm{~Hz}), 5.49(\mathrm{dd}, 1 \mathrm{H}, J=15.6,6.6 \mathrm{~Hz}), 4.34(\mathrm{~m}, 1 \mathrm{H}), 4.06(\mathrm{~m}, 4 \mathrm{H}), 3.96(\mathrm{~m}, 2 \mathrm{H})$, $3.87(\mathrm{~m}, 1 \mathrm{H}), 3.16(\mathrm{t}, \mathrm{br},-\mathrm{OH}), 2.35(\mathrm{~m}, 1 \mathrm{H}), 2.26(\mathrm{~m}, 1 \mathrm{H}), 2.16(\mathrm{~m}, 1 \mathrm{H}), 1.82(\mathrm{~m}, 1 \mathrm{H})$, $1.72(\mathrm{~m}, 1 \mathrm{H}), 1.57(\mathrm{~m}, 3 \mathrm{H}), 1.49(\mathrm{~m}, 7 \mathrm{H}), 1.46(\mathrm{~m}, 3 \mathrm{H}), 1.44(\mathrm{~s}, 3 \mathrm{H}), 1.41(\mathrm{~s}, 3 \mathrm{H}), 1.40$ (s, br, 6H), $1.370(\mathrm{~s}, 3 \mathrm{H}), 1.365$ (s, 3H), 1.341 (s, 3H), 1.325 (s, 3H), $1.02(\mathrm{~d}, 3 \mathrm{H}, J=6.6$ $\mathrm{Hz}), 0.92$ (dd, $6 \mathrm{H}, J=13.2,7.2 \mathrm{~Hz}) .{ }^{13} \mathrm{C} \mathrm{NMR}\left(\mathrm{CDCl}_{3}, 150 \mathrm{MHz}\right): \delta 142.1,135.0,131.2$, 100.5, 98.8 (3C), 98.7, 79.9, 70.5, 67.9, 65.6, 65.4, 65.2, 64.8, 63.1, 62.5, 43.2, 42.8, $42.4,42.2,39.4,38.8,38.0,37.9,36.0,30.7,30.4,30.50,30.46,30.28,24.8,24.7,20.0$ (2C), 19.9, 19.8, 17.3, 13.9. $[\alpha]_{\mathrm{D}}+11\left(\mathrm{c} 0.465, \mathrm{CDCl}_{3}\right)$.

Phosphonate ester of 50. To a solution of alcohol 50 (9.3 mg, $11.7 \mu \mathrm{mol})$ in $\mathrm{CH}_{2} \mathrm{Cl}_{2}$ $(0.5 \mathrm{~mL})$, DMAP $(6.2 \mathrm{mg}, 51.0 \mu \mathrm{mol})$, BOPPF $_{6}(21.3 \mathrm{mg}, 41.0 \mu \mathrm{mol})$ and diethylphosphonoacetic acid $(6.9 \mathrm{mg}, 35.0 \mu \mathrm{mol})$ were added. The reaction was stirred at room temperature for $22 \mathrm{~h}$ and concentrated. The phosphonate ester was isolated in $91 \%$ yield $(10 \mathrm{mg})$ after chromatography on silica gel pretreated with 2.5 volume $\%$ of $\mathrm{Et}_{3} \mathrm{~N}$ with pentane - ether $(3: 7)$.

${ }^{1} \mathrm{H} \mathrm{NMR}\left(\mathrm{CDCl}_{3}, 600 \mathrm{MHz}\right): \delta 6.53(\mathrm{~m}, 1 \mathrm{H}), 6.09(\mathrm{~d}, 1 \mathrm{H}, \mathrm{J}=14.4 \mathrm{~Hz}), 5.49(\mathrm{~m}, 2 \mathrm{H}), 4.71$ $(\mathrm{m}, 1 \mathrm{H}), 4.30(\mathrm{~m}, 1 \mathrm{H}), 4.17(\mathrm{~m}, 4 \mathrm{H}), 4.06(\mathrm{~m}, 4 \mathrm{H}), 3.96(\mathrm{~m}, 2 \mathrm{H}), 3.87(\mathrm{~m}, 1 \mathrm{H}), 2.97(\mathrm{~d}$, $\left.1 \mathrm{H}^{2} J_{P-H}=22.2 \mathrm{~Hz}\right), 2.45(\mathrm{~m}, 1 \mathrm{H}), 2.26(\mathrm{~m}, 1 \mathrm{H}), 2.16(\mathrm{~m}, 1 \mathrm{H}), 1.89-1.80(\mathrm{~m}, 2 \mathrm{H}), 1.57$ $(\mathrm{m}, 4 \mathrm{H}), 1.52-1.46(\mathrm{~m}, 9 \mathrm{H}), 1.43(\mathrm{~s}, 3 \mathrm{H}), 1.41(\mathrm{~s}, 3 \mathrm{H}), 1.40(\mathrm{~s}, 6 \mathrm{H}), 1.39(\mathrm{~s}, 3 \mathrm{H}), 1.34(\mathrm{t}$, $6 \mathrm{H}, J=7.2 \mathrm{~Hz}), 1.33(\mathrm{~s}, 3 \mathrm{H}), 1.32(\mathrm{~s}, 3 \mathrm{H}), 1.00(\mathrm{~d}, 3 \mathrm{H}, J=6.6 \mathrm{~Hz}), 0.88(\mathrm{~d}, 6 \mathrm{H}, J=6.6$ $\mathrm{Hz}) .{ }^{13} \mathrm{C} \mathrm{NMR}\left(\mathrm{CDCl}_{3}, 150 \mathrm{MHz}\right): \delta 165.8\left(\mathrm{~d},{ }^{2} J_{P-C}=25 \mathrm{~Hz}\right), 142.1,133.4,131.7,100.6$, 98.77 (2C), 98.75, 98.68, 82.5, 70.5, 67.9, 65.7, 65.4, 65.2, 64.8, 63.2, 62.7 (2C), 62.5, 43.2, 42.8, 42.4, 42.2, 38.8, 38.7, 38.0, 37.7, 36.0, 34.3 (d, ${ }^{1} \mathrm{~J}_{P-C}=536 \mathrm{~Hz}$ ), 30.5, 30.3, 29.7, 24.7, 20.0 (2C), 19.9, 19.8, 16.5 (2C), 16.4, 15.8. IR (neat, $\mathrm{cm}^{-1}$ ): 2990 (s), 2940 
(s), 2915 (s), 1730 (s), 1460 (w), 1380 (s), 1265 (s), 1225 (s), 1200 (s), 1170 (s), 1110 (s), 1050 (s), 1025 (s), 970 (s), 940 (s). [ $\alpha]_{\mathrm{D}}+13$ (c 0.525, $\mathrm{CDCl}_{3}$ ).

All-trans-7-(tributylstannyl)-hepta-2,4,6-trienoic acid, ethyl ester. To a solution of all-trans-5-(tributylstannyl)-2,4 -pentadien-1-ol ${ }^{7}\left(200 \mathrm{mg}, 0.54 \mathrm{mmol}\right.$ ) and $\mathrm{Et}_{3} \mathrm{~N}(0.4 \mathrm{~mL})$ in $\mathrm{CH}_{2} \mathrm{Cl}_{2}(3 \mathrm{~mL})$, a solution of $\mathrm{SO}_{3}$ pyridine complex $(260 \mathrm{mg}, 1.62 \mathrm{mmol})$ in DMSO (1.2 $\mathrm{mL}$ ) was added slowly at $0^{\circ} \mathrm{C}$. After addition, the reaction was stirred at ambient temperatures for $3 \mathrm{~h}$ and concentrated. The corresponding conjugated aldehyde (173 $\mathrm{mg}, 86 \%$ yield) was purified by flash chromatography on silica gel pretreated with 2.5 volume $\%$ of $\mathrm{Et}_{3} \mathrm{~N}$ with mixture of pentane - ether (95:5). Spectroscopic characteristics were identical to previously reported. ${ }^{7}$

This aldehyde (160 mg, $0.43 \mathrm{mmol}$ ) was dissolved in $\mathrm{CH}_{2} \mathrm{Cl}_{2}(5 \mathrm{~mL})$ and (carbethoxymethylene)triphenylphosphorane $(450 \mathrm{mg}, 1.29 \mathrm{mmol})$ was added. The reaction was stirred for 2 days, then concentrated and chromatographed on silica gel pretreated with 2.5 volume $\%$ of $\mathrm{Et}_{3} \mathrm{~N}$ with pentane - ether (95:5) yielding above ethyl ester in 95\% (184 mg).

Ethyl ester. ${ }^{1} \mathrm{H}$ NMR $\left(\mathrm{CDCl}_{3}, 600 \mathrm{MHz}\right): \delta 7.31(\mathrm{dd}, 1 \mathrm{H}, J=15.6,11.4 \mathrm{~Hz}), 6.63(\mathrm{~m}, 2 \mathrm{H})$, $6.51(\mathrm{dd}, 1 \mathrm{H}, J=15.0,11.4 \mathrm{~Hz}), 6.25(\mathrm{dd}, 1 \mathrm{H}, J=14.4,11.4 \mathrm{~Hz}), 5.90(\mathrm{~d}, 1 \mathrm{H}, J=15.0$ $\mathrm{Hz}), 4.21(\mathrm{q}, 2 \mathrm{H}, J=7.8 \mathrm{~Hz}), 1.51(\mathrm{~m}, 6 \mathrm{H}), 1.31(\mathrm{~m}, 12 \mathrm{H}), 0.94(\mathrm{t}, 3 \mathrm{H}, J=7.8 \mathrm{~Hz}), 0.90$ $(\mathrm{t}, 9 \mathrm{H}, J=7.2 \mathrm{~Hz}) \cdot{ }^{13} \mathrm{C} \mathrm{NMR}\left(\mathrm{CDCl}_{3}, 150 \mathrm{MHz}\right): \delta 167.2,146.0,144.9,143.1,142.3$, 128.8, 121.4, 60.4, 29.2, 27.4, 14.5, 13.9, 9.8. IR (neat, $\mathrm{cm}^{-1}$ ): 2955 (s), 2920 (s), 2850 (m), $1715(\mathrm{~s}), 1620(\mathrm{~s}), 1460(\mathrm{~m}), 1365(\mathrm{~m}), 1325(\mathrm{~m}), 1240(\mathrm{~m}), 1200(\mathrm{~m}), 1540(\mathrm{~m})$, 1130 (s), 1010 (m), 845 (w). HRMS (FAB+): Calcd. for $\mathrm{C}_{21} \mathrm{H}_{39} \mathrm{O}_{2} \mathrm{Sn}^{120}\left([\mathrm{M}+\mathrm{H}]^{+}\right)$, 443.1972. Found, 443.1960.

All-trans-7-(tributylstannyl)-2,4,6-heptatrien-1-ol. To a solution of all-trans-7(tributylstannyl)-hepta-2,4,6-trienoic acid, ethyl ester (90 mg, $0.2 \mathrm{mmol}$ ) in $\mathrm{CH}_{2} \mathrm{Cl}_{2}$ (2 $\mathrm{mL}), 1 \mathrm{M}$ solution of DIBAL-H in $\mathrm{CH}_{2} \mathrm{Cl}_{2}(600 \mu \mathrm{L}, 0.6 \mathrm{mmol})$ was added at $-78^{\circ} \mathrm{C}$. The reaction was stirred for $50 \mathrm{~min}$ at $-78^{\circ} \mathrm{C}$ then diluted with AcOEt $(2 \mathrm{~mL})$ and allowed to

7. Dominguez, B.; Iglesias, B.; Lera, A. R. Tetrahedron 1999, 55, 15071. 
warm to room temperature. The mixture was poured into saturated aqueous potassium sodium tartrate and extracted with $\mathrm{CH}_{2} \mathrm{Cl}_{2}$. Organic fractions were dried with $\mathrm{MgSO}_{4}$, filtered and concentrated. The labile triene-alcohol was isolated in $75 \%$ yield $(60 \mathrm{mg})$ after flash chromatography on silica gel pretreated with 2.5 volume $\%$ of $\mathrm{Et}_{3} \mathrm{~N}$ with mixture of pentane - ether $(7: 3)$. This compound was protected from light, stored in a refrigerator and used in following reactions within two weeks.

${ }^{1} \mathrm{H} \mathrm{NMR}\left(\mathrm{CDCl}_{3}, 600 \mathrm{MHz}\right): \delta 6.57(\mathrm{dd}, 1 \mathrm{H}, J=18.0,9.6 \mathrm{~Hz}), 6.33-6.27(\mathrm{~m}, 2 \mathrm{H}), 6.22-$ $6.19(\mathrm{~m}, 2 \mathrm{H}), 5.88(\mathrm{~m}, 1 \mathrm{H}), 4.22(\mathrm{t}, 2 \mathrm{H}, J=5.4 \mathrm{~Hz}), 1.51(\mathrm{~m}, 6 \mathrm{H}), 1.32(\mathrm{~m}, 6 \mathrm{H}), 0.92-$ $0.88(\mathrm{~m}, 15 \mathrm{H}) .{ }^{13} \mathrm{C} \mathrm{NMR}\left(\mathrm{CDCl}_{3}, 150 \mathrm{MHz}\right): \delta 146.7,136.3,136.0,132.6,131.8,130.8$, 63.7, 29.3, 27.5, 13.9, 9.7. IR (neat, $\mathrm{cm}^{-1}$ ): 3305 (m, br), 2955 (s), 2920 (s), 2852 (s), $1605(\mathrm{w}), 1550(\mathrm{w}), 1460(\mathrm{~m}), 1375(\mathrm{~m}), 1290(\mathrm{w}), 1180(\mathrm{w}), 1080(\mathrm{~m}), 1000$ (s), 870 (w). HRMS (FAB+): Calcd. for $\mathrm{C}_{19} \mathrm{H}_{36} \mathrm{OSn}^{120} \mathrm{Li}\left([\mathrm{M}+\mathrm{Li}]^{+}\right)$, 407.1948. Found, 407.1961.

Polyene 51. All-trans-7-(tributylstannyl)-2,4,6-heptatrien-1-ol (16.1 mg, $40.0 \mu \mathrm{mol})$, i$\operatorname{Pr}_{2}$ NEt $(7 \mu \mathrm{L}, 40.0 \mu \mathrm{mol})$ and $\mathrm{Ph}_{3}$ As $(6.5 \mathrm{mg}, 20.0 \mu \mathrm{mol})$ were dissolved in THF (250 $\mu \mathrm{L}$ ). The reaction flask was wrapped in aluminum foil, and a solution of iodide $\mathbf{5 0}$ (10.0 $\mathrm{mg}, 10.0 \mu \mathrm{mol})$ in THF $(250 \mu \mathrm{L})$ and a solution of $\mathrm{Pd}_{2}(\mathrm{dba})_{3} \mathrm{CHCl}_{3}(2.0 \mathrm{mg}, 2.0 \mu \mathrm{mol})$ in THF $(250 \mu \mathrm{L})$ were added simultaneously over $2 \mathrm{~h}$. The reaction was stirred at ambient temperature for $20 \mathrm{~h}$, then concentrated and chromatographed on silica gel pretreated with 2.5 volume $\%$ of $\mathrm{Et}_{3} \mathrm{~N}$ with mixture of pentane - ether $(1: 1 \rightarrow 3: 7)$ and then with straight ether. $2.7 \mathrm{mg} \mathrm{(27 \% )}$ of starting iodide $\mathbf{5 0}$ was recovered along with $3.1 \mathrm{mg}$ (32\%) of desired polyene alcohol 56.

${ }^{1} \mathrm{H}$ NMR $\left(\mathrm{CDCl}_{3}, 600 \mathrm{MHz}\right): \delta 6.32-6.27(\mathrm{~m}, 2 \mathrm{H}), 6.25-6.11(\mathrm{~m}, 4 \mathrm{H}), 5.85(\mathrm{~m}, 1 \mathrm{H}), 5.71$ (m, 1H), $5.50(\mathrm{~m}, 2 \mathrm{H}), 4.72(\mathrm{dd}, 1 \mathrm{H}, J=7.8,4.8 \mathrm{~Hz}), 4.30(\mathrm{~m}, 1 \mathrm{H}), 4.21(\mathrm{~m}, 2 \mathrm{H}), 4.18$ $(\mathrm{m}, 4 \mathrm{H}), 4.11-4.03(\mathrm{~m}, 4 \mathrm{H}), 3.96(\mathrm{~m}, 2 \mathrm{H}), 386(\mathrm{~m}, 1 \mathrm{H}), 2.98\left(\mathrm{dd}, 2 \mathrm{H},{ }^{3} J_{\mathrm{H}-\mathrm{H}}=1.2 \mathrm{~Hz},{ }^{2} J_{\mathrm{P}-}\right.$ $\mathrm{H}=21 \mathrm{~Hz}), 2.46(\mathrm{~m}, 1 \mathrm{H}), 2.35(\mathrm{~m}, 1 \mathrm{H}), 2.25-2.18(\mathrm{~m}, 2 \mathrm{H}), 1.89-1.80(\mathrm{~m}, 3 \mathrm{H}), 1.58(\mathrm{~m}$, $5 \mathrm{H}), 1.52-1.47(\mathrm{~m}, 6 \mathrm{H}), 1.44(\mathrm{~s}, 6 \mathrm{H}), 1.42(\mathrm{~s}, 3 \mathrm{H}), 1.401(\mathrm{~s}, 3 \mathrm{H}), 1.395(\mathrm{~s}, 3 \mathrm{H}), 1.38(\mathrm{~s}$, $3 \mathrm{H}), 1.36(\mathrm{~s}, 3 \mathrm{H}), 1.35(\mathrm{~s}, 3 \mathrm{H}), 1.32(\mathrm{~s}, \mathrm{br}, 3 \mathrm{H}), 1.00(\mathrm{~d}, 3 \mathrm{H}, \mathrm{J}=6.6 \mathrm{~Hz}), 0.89(\mathrm{~d}, 6 \mathrm{H}, 6.6$ $\mathrm{Hz})$. 
Macrolactone 52. To a solution of alcohol $51(3.0 \mathrm{mg}, 3.0 \mu \mathrm{mol})$ in $\mathrm{CH}_{2} \mathrm{Cl}_{2}(250 \mu \mathrm{L})$, crystalline $\mathrm{NaHCO}_{3}(6.3 \mathrm{mg}, 75.0 \mu \mathrm{mol})$ was added, followed by addition of DessMartin periodinane $(2.6 \mathrm{mg}, 6.0 \mu \mathrm{mol})$. The reaction was protected from light and stirred for $2.5 \mathrm{~h}$, then diluted with mixture of saturated $\mathrm{NaHCO}_{3}(\mathrm{aq})$ and water $(3: 1,0.2 \mathrm{~mL})$ and extracted with ether. Organic fractions were dried with $\mathrm{Na}_{2} \mathrm{SO}_{4}$, decanted and concentrated yielding crude aldehyde as 1: 1 mixture of two isomers.

${ }^{1} \mathrm{H} \mathrm{NMR}\left(\mathrm{CDCl}_{3}, 600 \mathrm{MHz}\right): \delta 9.58(\mathrm{~d}, 0.5 \mathrm{H}, J=8.4 \mathrm{~Hz}), 9.57(\mathrm{~d}, 0.5 \mathrm{H}, J=8.4 \mathrm{~Hz}), 7.24-$ $7.12(\mathrm{~m}, 2 \mathrm{H}), 6.74-6.67(\mathrm{~m}, 1 \mathrm{H}), 6.52-6.39(\mathrm{~m}, 2 \mathrm{H}), 6.28-6.14(\mathrm{~m}, 2 \mathrm{H}), 5.90(\mathrm{~m}, 1 \mathrm{H})$, $5.50(\mathrm{~m}, 2 \mathrm{H}), 4.72(\mathrm{~m}, 1 \mathrm{H}, J=7.2,4.8 \mathrm{~Hz}), 4.30(\mathrm{~m}, 1 \mathrm{H}), 4.18(\mathrm{p}, 4 \mathrm{H}, J=6.6 \mathrm{~Hz}), 4.10-$ $4.02(\mathrm{~m}, 4 \mathrm{H}), 3.97(\mathrm{~m}, 2 \mathrm{H}), 3.91(\mathrm{~m}, 1 \mathrm{H}), 2.98\left(\mathrm{~d}, 2 \mathrm{H},{ }^{2} \mathrm{JP}_{\mathrm{H}}=21.6 \mathrm{~Hz}\right), 2.55(\mathrm{dd}, 1 \mathrm{H}, J=$ 14.4, $5.4 \mathrm{~Hz}), 2.46(\mathrm{~m}, 1 \mathrm{H}), 2.38(\mathrm{~m}, 1 \mathrm{H}), 2.28(\mathrm{~m}, 1 \mathrm{H}), 1.90-1.81(\mathrm{~m}, 3 \mathrm{H}), 1.59(\mathrm{~m}$, $5 \mathrm{H}), 1.49(\mathrm{~m}, 6 \mathrm{H}), 1.44(\mathrm{~s}, 6 \mathrm{H}), 1.43(\mathrm{~s}, 3 \mathrm{H}), 1.403(\mathrm{~s}, 3 \mathrm{H}), 1.396(\mathrm{~s}, 3 \mathrm{H}), 1.387(\mathrm{~s}, 3 \mathrm{H})$, $1.36(\mathrm{~s}, 3 \mathrm{H}), 1.35$ (s, 3H), 1.32 (s, br, 3H), $1.26(\mathrm{~s}, \mathrm{br}, 3 \mathrm{H}), 1.00$ (d, 3H, J= 7.2 Hz), 0.89 $(\mathrm{d}, 6 \mathrm{H}, J=6.6 \mathrm{~Hz})$.

$\mathrm{LiCl}(3.1 \mathrm{mg}, 75.0 \mu \mathrm{mol})$ was dried under vacuum at $145{ }^{\circ} \mathrm{C}$ for several hours, then cooled to room temperature and combined with an acetonitrile $(2.4 \mathrm{~mL})$ solution of crude aldehyde obtained by oxidation of $\mathbf{5 1}$ (ca. $3.0 \mu \mathrm{mol}$ ). The reaction was shielded from light and stirred for $30 \mathrm{~min}$, then DBU ( $23 \mu \mathrm{L}, 150.0 \mu \mathrm{mol})$ was added. The mixture was stirred for 2 days, diluted with $\mathrm{pH} 7$ phosphate buffer $(1 \mathrm{~mL})$ and extracted with ether. Organic fractions were dried with $\mathrm{Na}_{2} \mathrm{SO}_{4}$, decanted, concentrated and chromatographed on silica gel pretreated with 2.5volume\% of $\mathrm{Et}_{3} \mathrm{~N}$ with mixture of pentane - ether (6: 4). $0.7 \mathrm{mg}$ (29\% from alcohol 51 ) of macrocyclic tetracetonide 52 was isolated $\left(R_{f}, 0.33\right.$, pentane - ether, $\left.6: 4\right)$.

${ }^{1} \mathrm{H}$ NMR $\left(\mathrm{CDCl}_{3}, 600 \mathrm{MHz}\right): \delta 3.35(\mathrm{dd}, 1 \mathrm{H}, J=15.6,12.0 \mathrm{~Hz}), 6.58(\mathrm{dd}, 1 \mathrm{H}, J=14.4$, $10.8 \mathrm{~Hz}), 6.39-6.35(\mathrm{~m}, 2 \mathrm{H}), 6.32-6.23(\mathrm{~m}, 3 \mathrm{H}), 6.17-6.13(\mathrm{~m}, 1 \mathrm{H}), 5.81(\mathrm{~d}, 1 \mathrm{H}, J=$ $15.6 \mathrm{~Hz}$ ), 5.65- $5.59(\mathrm{~m}, 2 \mathrm{H}), 5.41(\mathrm{dd}, 1 \mathrm{H}, J=15.6,4.2 \mathrm{~Hz}), 4.83(\mathrm{dd}, 1 \mathrm{H}, J=9.6,3.0$ $\mathrm{Hz}), 4.30(\mathrm{~m}, 1 \mathrm{H}), 4.04(\mathrm{~m}, 1 \mathrm{H}), 3.97(\mathrm{~m}, 3 \mathrm{H}), 3.90(\mathrm{~m}, 1 \mathrm{H}), 3.84(\mathrm{~m}, 1 \mathrm{H}), 3.77(\mathrm{~m}, 1 \mathrm{H})$, 2.62- $2.56(\mathrm{~m}, 4 \mathrm{H}), 1.92-1.89(\mathrm{~m}, 3 \mathrm{H}), 1.56(\mathrm{~m}, 5 \mathrm{H}), 1.48(\mathrm{~m}, 6 \mathrm{H}), 1.45(\mathrm{~s}, 3 \mathrm{H}), 1.40(\mathrm{~s}$, $3 \mathrm{H}), 1.39(\mathrm{~s}, 3 \mathrm{H}), 1.38(\mathrm{~s}, 6 \mathrm{H}), 1.35(\mathrm{~s}, 3 \mathrm{H}), 1.29(\mathrm{~s}, 3 \mathrm{H}), 1.18(\mathrm{~s}, 3 \mathrm{H}), 1.03(\mathrm{~d}, 3 \mathrm{H}, J=$ $6.6 \mathrm{~Hz}), 0.94(\mathrm{~d}, 3 \mathrm{H}, J=7.2 \mathrm{~Hz}), 0.90(\mathrm{~d}, 3 \mathrm{H}, J=6.6 \mathrm{~Hz})$. 
Synthetic RK-397 (4). To a solution of macrocyclic tetracetonide 52 (0.7 mg, $0.86 \mu \mathrm{mol})$ in methanol $(200 \mu \mathrm{L})$ Dowex $\circledast 50 \mathrm{~W} \times 2$ acidic resin $(2 \mathrm{mg})$ was added. The reaction was vigorously stirred for a day, then decanted concentrated and chromatographed on silica gel pretreated with 2.5 volume $\%$ of $\mathrm{Et}_{3} \mathrm{~N}$ with mixture of methanol - ethylacetate $(2: 8)$ producing ca. $0.4 \mathrm{mg}$ (ca. $73 \%$ yield) of synthetic RK-397 (4).

${ }^{1} \mathrm{H}$ NMR $\left(\mathrm{CDCl}_{3}, 600 \mathrm{MHz}\right): \delta 7.30(\mathrm{dd}, 1 \mathrm{H}, J=15.0,11.4 \mathrm{~Hz}), 6.69(\mathrm{dd}, 1 \mathrm{H}, J=14.4$, $10.8 \mathrm{~Hz}), 6.53-6.35(\mathrm{~m}, 4 \mathrm{H}), 6.31-6.20(\mathrm{~m}, 2 \mathrm{H}), 5.89(\mathrm{~d}, 1 \mathrm{H}, J=15.6 \mathrm{~Hz}), 5.85(\mathrm{~m}, 1 \mathrm{H})$, $5.64(\mathrm{dd}, 1 \mathrm{H}, J=15.6,4.8 \mathrm{~Hz}), 5.52(\mathrm{dd}, 1 \mathrm{H}, J=15.6,4.8 \mathrm{~Hz}), 4.21(\mathrm{~m}, 1 \mathrm{H}), 4.05(\mathrm{~m}$, 2H), 3.95- $3.90(\mathrm{~m}, 4 \mathrm{H}), 3.81(\mathrm{~m}, 2 \mathrm{H}), 2.64(\mathrm{~m}, 2 \mathrm{H}), 2.14(\mathrm{t}, 1 \mathrm{H}, J=6.6 \mathrm{~Hz}), 1.92(\mathrm{~m}$, 1H), $1.64(\mathrm{~m}, 2 \mathrm{H}), 1.45(\mathrm{~m}, 2 \mathrm{H}), 1.39(\mathrm{~m}, 1 \mathrm{H}), 1.35(\mathrm{~s}, \mathrm{br}, 3 \mathrm{H}), 1.31(\mathrm{~s}, \mathrm{br}, 3 \mathrm{H}), 1.28(\mathrm{~s}$, br, $3 \mathrm{H}), 1.06(\mathrm{~d}, 3 \mathrm{H}, J=6.6 \mathrm{~Hz}), 0.97(\mathrm{~d}, 3 \mathrm{H}, J=6.6 \mathrm{~Hz}), 0.88(\mathrm{~d}, 3 \mathrm{H}, J=6.6 \mathrm{~Hz}$ ).

Circular dichroism in $\mathrm{MeOH}$ : synthetic RK-397 showed a negative Cotton effect $\left(\lambda_{\max }\right.$, $210 \mathrm{~nm} ; \lambda_{\min }, 260 \mathrm{~nm}$ ). UV (MeOH, $\left.\lambda_{\max }\right): 360 \mathrm{~nm}$. HRMS (MALDI- DHB): m/z 659.38 $\left([\mathrm{M}+\mathrm{Na}]^{+}\right)$. 


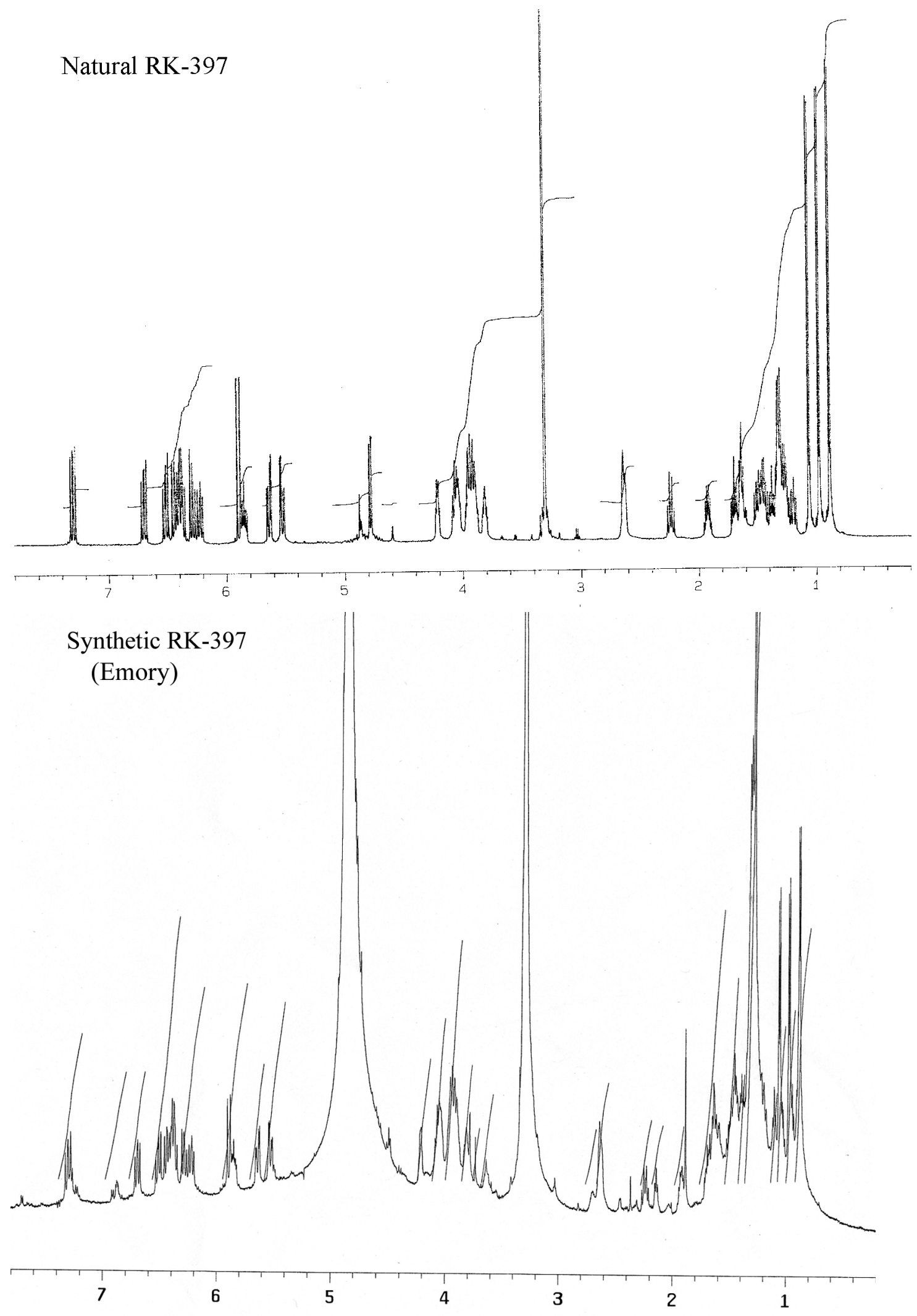

Figure i. ${ }^{1} \mathrm{H}$ NMR spectra comparison for synthetic and natural RK-397. 

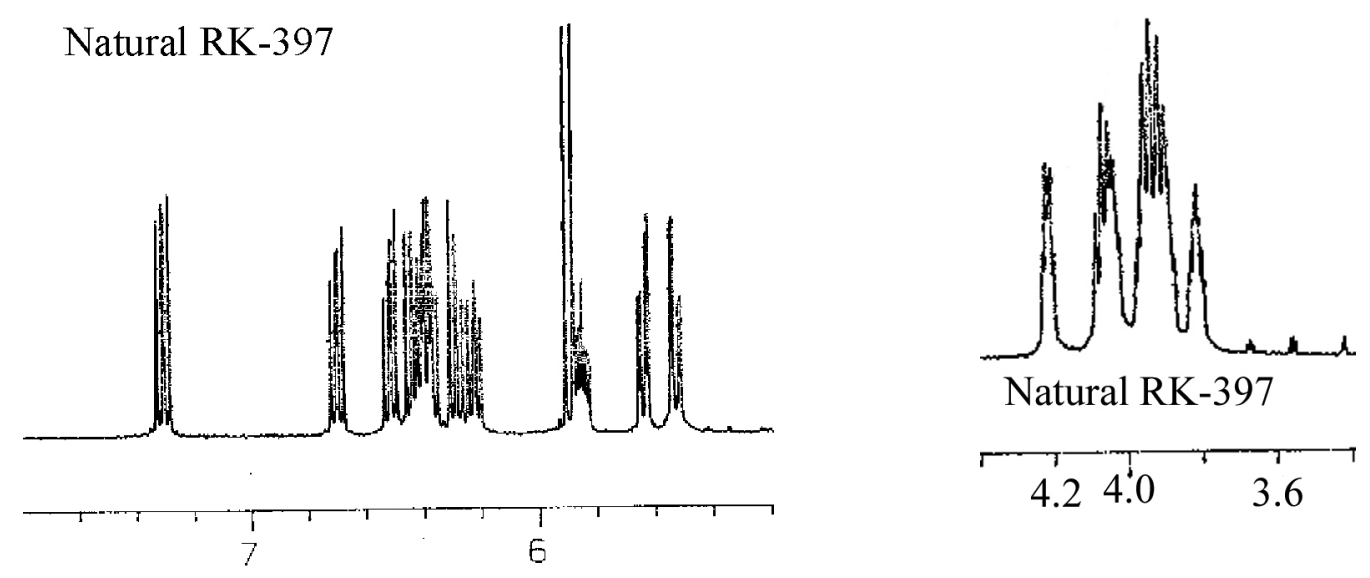

Synthetic RK-397
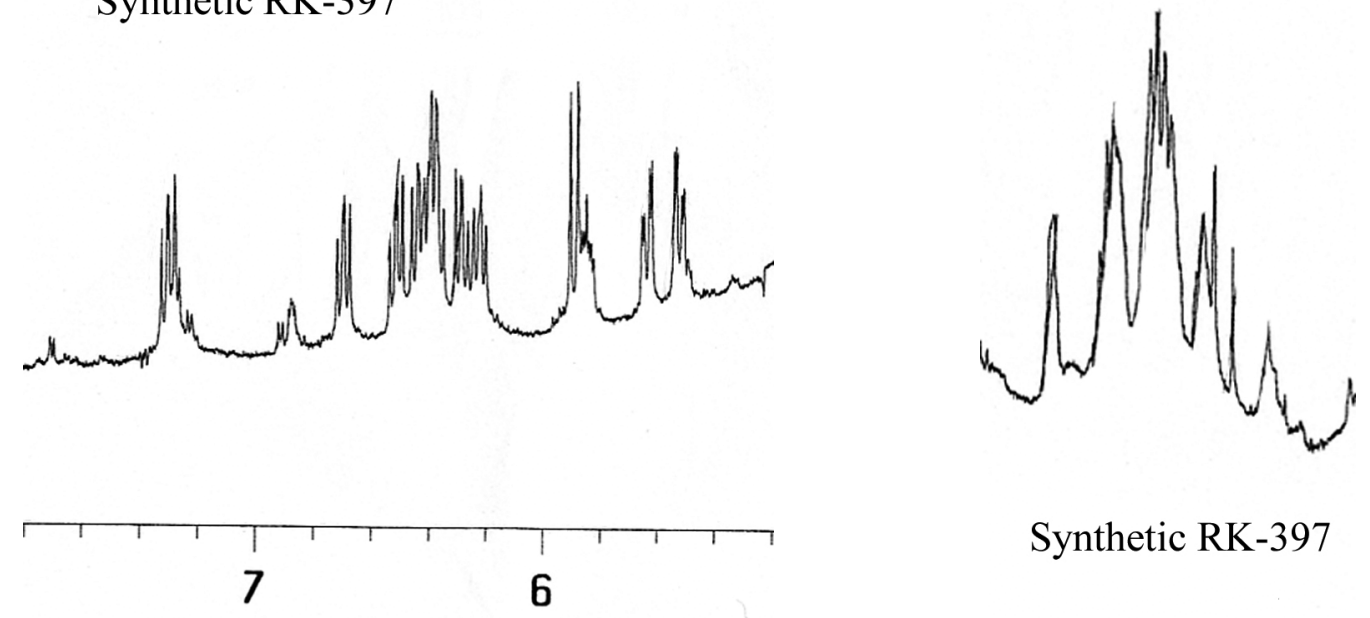

Synthetic RK-397

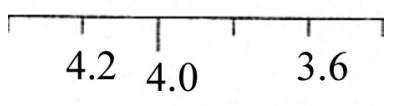

Figure ii. ${ }^{1} \mathrm{H}$ NMR spectra comparison for synthetic and natural RK-397: 7.8- 5.2 ppm and 4.4-3.4 ppm regions. 

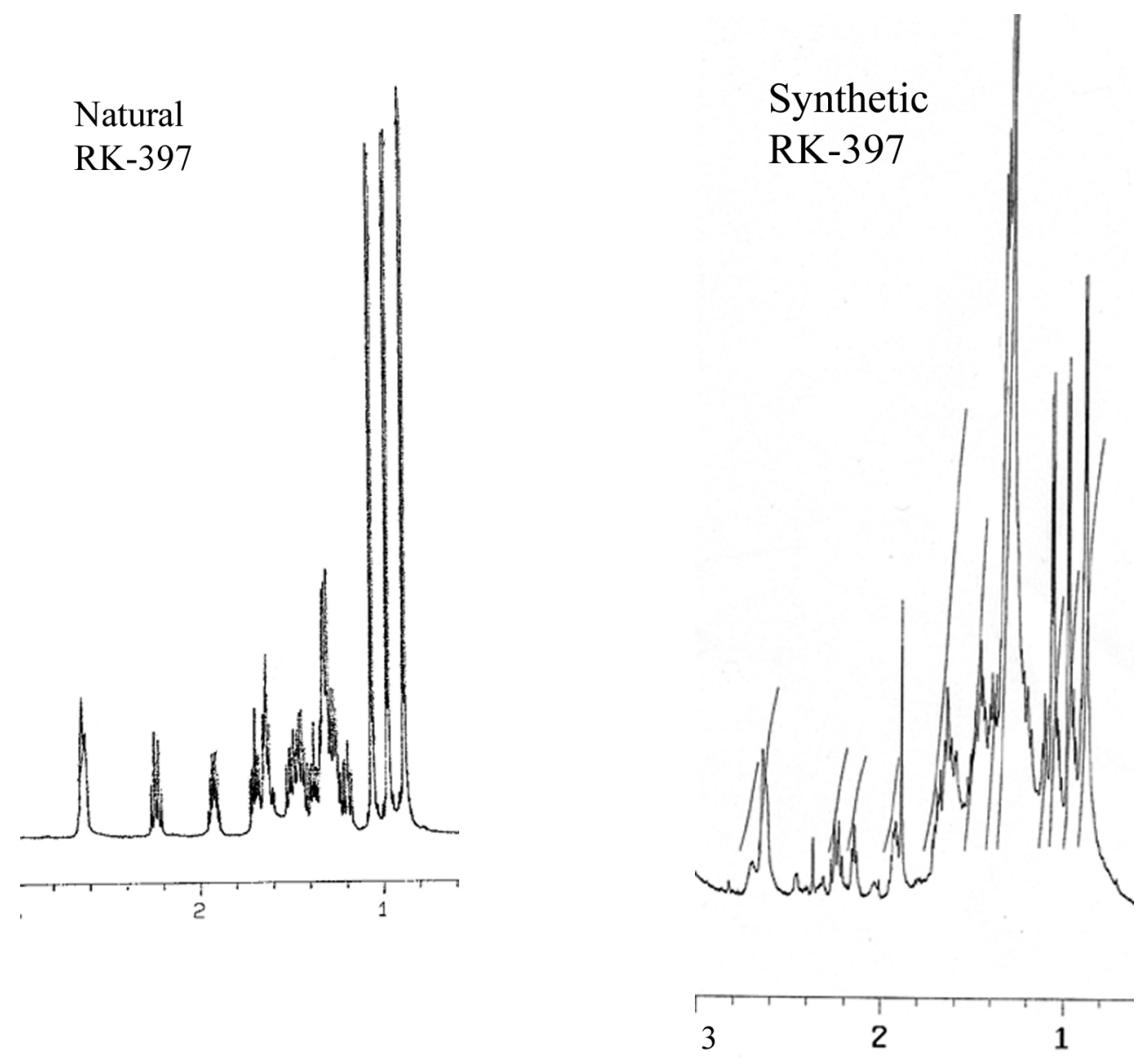

Figure iii. ${ }^{1} \mathrm{H}$ NMR spectra comparison for synthetic and natural RK-397: 3.0-0.8 ppm region. 
Synthetic RK-397 (Circular Dichroism in $\mathrm{MeOH}$ )

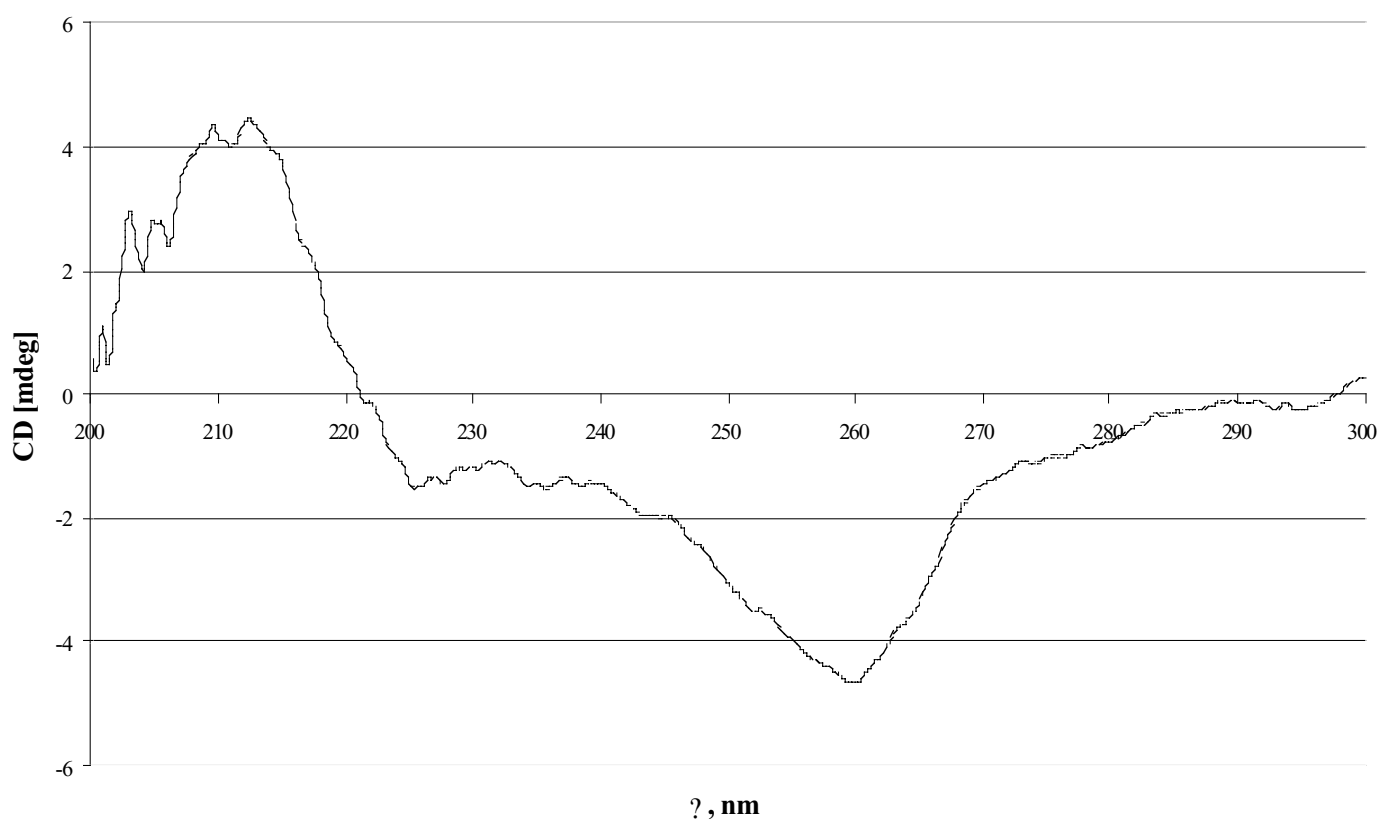

Figure iv. Circular dichroism spectra of synthetic RK-397 (4) in methanol. 

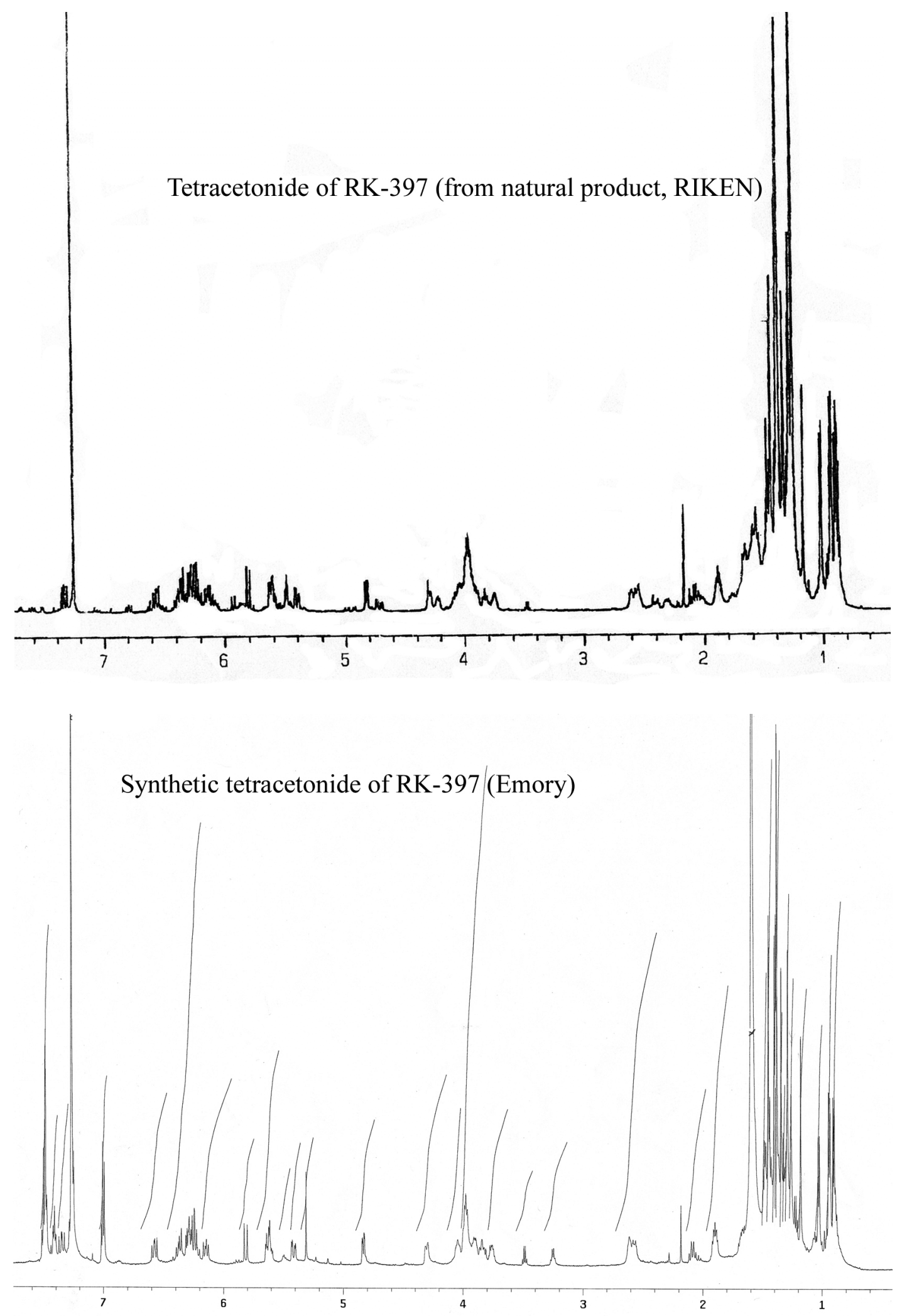

Figure v. ${ }^{1} \mathrm{H}$ NMR spectra comparison for natural and synthetic RK-397 tetracetonide 52. 